\title{
Study of Extra Material in Front of the ATLAS \\ Electromagnetic Barrel Calorimeter using High- $p_{T}$ \\ Electrons
}

by

\section{Kathleen Whalen}

\author{
A thesis submitted to the \\ Faculty of Graduate Studies and Research \\ in partial fulfilment of the requirements \\ for the degree of \\ Master of Science \\ Department of Physics \\ Carleton University \\ Ottawa-Carleton Institute for Physics \\ Ottawa, Ontario \\ September 2009 \\ (C)Kathleen Whalen, 2009
}




\author{
Library and Archives \\ Canada \\ Published Heritage \\ Branch \\ 395 Wellington Street \\ Ottawa ON K1A ON4 \\ Canada
}

Bibliothèque et

Archives Canada

Direction du

Patrimoine de l'édition

395, rue Wellington

Ottawa ON K1A ON4

Canada
Your file Votre référence
ISBN: 978-0-494-60213-3
Our file Notre réference
ISBN: $978-0-494-60213-3$
NOTICE:

The author has granted a nonexclusive license allowing Library and Archives Canada to reproduce, publish, archive, preserve, conserve, communicate to the public by telecommunication or on the Internet, loan, distribute and sell theses worldwide, for commercial or noncommercial purposes, in microform, paper, electronic and/or any other formats.

The author retains copyright ownership and moral rights in this thesis. Neither the thesis nor substantial extracts from it may be printed or otherwise reproduced without the author's permission.
AVIS:

L'auteur a accordé une licence non exclusive permettant à la Bibliothèque et Archives Canada de reproduire, publier, archiver, sauvegarder, conserver, transmettre au public par télécommunication ou par l'internet, prêter, distribuer et vendre des thèses partout dans le monde, à des fins commerciales ou autres, sur support microforme, papier, électronique et/ou autres formats.

L'auteur conserve la propriété du droit d'auteur et des droits moraux qui protège cette thèse. Ni la thèse ni des extraits substantiels de celle-ci ne doivent être imprimés ou autrement reproduits sans son autorisation.
In compliance with the Canadian Privacy Act some supporting forms may have been removed from this thesis.

While these forms may be included in the document page count, their removal does not represent any loss of content from the thesis.
Conformément à la loi canadienne sur la protection de la vie privée, quelques formulaires secondaires ont étè enlevés de cette thèse.

Bien que ces formulaires aient inclus dans la pagination, il n'y aura aucun contenu manquant. 


\section{Abstract}

The ATLAS electromagnetic barrel calorimeter is a lead-liquid argon sampling calorimeter that measures the energy of electrons, positrons and photons produced as a result of proton-proton collisions at CERN's Large Hadron Collider. During early operations, electromagnetic objects from well-known processes, including electrons from the decay $W \rightarrow e \nu$, will be used to calibrate this detector. It is important to identify the location and amount of material in front of the calorimeter, as this will influence its energy calibration. A method has been developed to determine the calorimeter's sensitivity to extra material using simulated electrons from $W \rightarrow e \nu$

decays. With $160 \mathrm{pb}^{-1}$, obtainable within two years at $10 \mathrm{TeV}$, the calorimeter is sensitive to a material increase of approximately $5 \%$ of a radiation length, for electron energies of up to $100 \mathrm{GeV}$. The validity of using the location of bremsstrahlung in the inner detector to pinpoint the location of extra material is also investigated. 


\section{Acknowledgements}

Many thanks to Dr. Manuella Vincter, for being such a patient, supportive and enthusiastic supervisor and teacher, despite the geographical separation, and for all the midnight e-mail conversations.

To my ATLAS officemates at Carleton, both past and present, thanks for being such a helpful and fun group to work with. The computing tips and the coffeemaker are equally appreciated.

To my parents, thanks for your unwavering support, even if you're not entirely sure of exactly what it is that I do.

Finally, to Pierre-Luc, thank you for your love and friendship, for putting up with me when I get frustrated, and for always having a solution for the most obscure ROOT bugs. 


\section{Contents}

$\begin{array}{ll}\text { Abstract } & \text { ii }\end{array}$

$\begin{array}{ll}\text { Acknowledgements } & \text { iii }\end{array}$

List of Tables $\quad$ vi

List of Figures $\quad$ vii

1 Introduction $\quad 1$

1.1 The Standard Model . . . . . . . . . . . . . . . . . . . . . . 1

1.2 Physics Studies at ATLAS . . . . . . . . . . . . . 6

1.3 Motivation for this Work . . . . . . . . . . . 7

2 The LHC and ATLAS $\quad 10$

2.1 The Large Hadron Collider . . . . . . . . . . . . . . . . . . 10

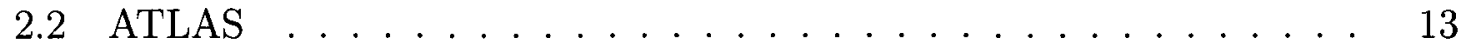

2.2 .1 ATLAS Coordinate System . . . . . . . . . . . . 14

2.2 .2 Inner Detector. . . . . . . . . . . . . . . . . . 15

2.2 .3 Calorimeters . . . . . . . . . . . . . . . . . . 17

2.2 .4 Muon Spectrometer . . . . . . . . . . . . . 20

2.2 .5 Trigger . . . . . . . . . . . . . . . . 21

3 The ATLAS Electromagnetic Calorimeter 2

3.1 Electromagnetic Showers . . . . . . . . . . . . . . . . . . . 24

3.1.1 Energy Loss of Electrons and Photons . . . . . . . . . . 24

3.1.2 Longitudinal Development of Electromagnetic Showers . . . . 27

3.1.3 Transverse Development of Electromagnetic Showers . . . . 30

3.2 Introduction to Electromagnetic Calorimetry . . . . . . . . . . . . 31

3.3 Performance Requirements . . . . . . . . . . . . . . . . . 33

3.4 Electromagnetic Calorimeter Design . . . . . . . . . . . . . . . 34 
3.5 Energy Reconstruction . . . . . . . . . . . . . . . . . 43

4 Impact of Extra Material in Front of the EM Calorimeter and in the Inner Detector $\quad 51$

4.1 Detector Geometry and Simulation of Events . . . . . . . . . . . 55

4.1 .1 Detector Geometry . . . . . . . . . . . . . . 55

$4.1 .2{\text { High- } \boldsymbol{p}_{\boldsymbol{T}} \text { Electrons } \ldots \ldots \ldots \ldots \ldots}_{57} \ldots \ldots \ldots \ldots \ldots$

4.2 Energy Reconstruction . . . . . . . . . . . . . . . . . . 63

4.3 Longitudinal Energy Deposition . . . . . . . . . . . . . . . . . 68

4.3.1 Impact of Extra Material in the Cryostat . . . . . . . . . 71

4.3.2 Estimating the Sensitivity of Longitudinal Energy Loss Variables to Extra Material . . . . . . . . . . . . . . 75

4.3.3 Attempt to Find a New Sensitive Shower Variable . . . . . . 83

4.3.4 Effect of Extra Material Between the Presampler and the First Sampling Layer . . . . . . . . . . . . . . 89

4.3.5 Feasibility With Early Data . . . . . . . . . . . . . 91

4.4 Impact of Bremsstrahlung in the Inner Detector . . . . . . . . . . . . 93

4.4 .1 Impact on Electron $\boldsymbol{E} / \boldsymbol{p} \ldots \ldots \ldots \ldots \ldots$

4.4.2 Impact on Energy Deposited in the Calorimeter . . . . . . . . 99

4.5 Summary . . . . . . . . . . . . . . . . 103

$\begin{array}{llr}5 & \text { Summary and Conclusions } & 105\end{array}$

$\begin{array}{lr}\text { Bibliography } & 110\end{array}$ 


\section{List of Tables}

1.1 The four forces of nature $\ldots \ldots \ldots \ldots \ldots$

3.1 Granularity and $\eta$ coverage of the electromagnetic barrel calorimeter readout ............................ 39

3.2 Granularity and $\eta$ coverage of the electromagnetic endcap calorimeter readout . . . . . . . . . . . . . . . . 42

4.1 Summary of material added in front of the electromagnetic barrel

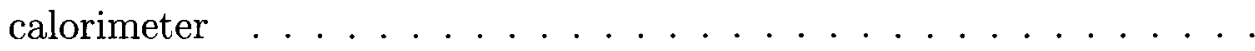

4.2 Summary of distorted and reference regions in the CSC-01-02-00 geometry . . . . . . . . . . . . . . . . . 60

4.3 Limits of integration over each layer of the electromagnetic barrel

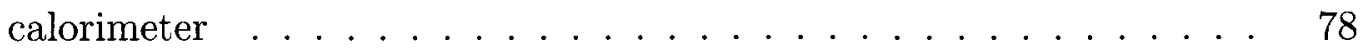

4.4 Approximate location in $R$ of extra inner detector material . . . . 100 


\section{List of Figures}

2.1 The LHC ring . . . . . . . . . . . . . . . . . . . . . 11

2.2 Cutaway view of ATLAS . . . . . . . . . . . . 13

2.3 Cutaway view of the inner detector $\ldots \ldots \ldots \ldots \ldots$

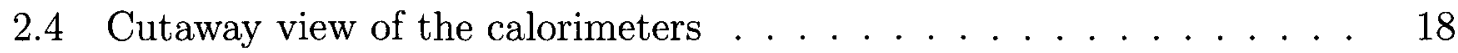

2.5 Cutaway view of the muon system . . . . . . . . . . . . 21

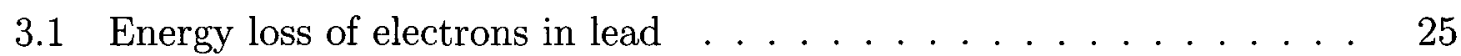

3.2 Photon cross sections in lead . . . . . . . . . . . . . 26

3.3 Electromagnetic shower . . . . . . . . . . . . . . . . . . . . 29

3.4 Longitudinal energy loss profile of a $35 \mathrm{GeV}$ electron in lead . . . . 31

3.5 Close-up of the absorbers and liquid argon gaps . . . . . . . . . 36

3.6 The four types of electrodes used in the electromagnetic calorimeter . 37

3.7 Electromagnetic barrel calorimeter module . . . . . . . . . . . . 38

3.8 Material distribution in the electromagnetic calorimeter . . . . . . . 40

3.9 A simulated electromagnetic shower in the accordion . . . . . . . . 44

3.10 Fitted longitudinal weights for electrons and photons . . . . . . . 46

3.11 Energy response of the electromagnetic calorimeter . . . . . . . . 48

4.1 Dependence of the energy resolution and the sampling term on $|\eta| \ldots \quad 53$

4.2 Energy loss of electrons in the inner detector . . . . . . . . . . . 54

4.3 Inner detector material distribution as a function of $\eta \ldots \ldots . . .56$

4.4 Nominal inner detector layout in $R-z \ldots \ldots \ldots \ldots$. . . . 57

4.5 Extra inner detector material distribution in $R-z \ldots \ldots \ldots$

4.6 Electromagnetic barrel calorimeter material distribution (in number of radiation lengths) as a function of $\eta \ldots \ldots \ldots \ldots$

4.7 Schematic of the extra material distribution . . . . . . . . . . 59

$4.8 p_{T}$ distribution of the simulated electron sample $\ldots \ldots \ldots \ldots 1$

$4.9 E$ distribution of the simulated electron sample $\ldots \ldots \ldots 2$

$4.10 \eta$ distribution of the simulated electron sample . . . . . . . . . 62

$4.11 \phi$ distribution of the simulated electron sample . . . . . . . . 63 
4.12 Relative difference between reconstructed and true electron energy . . 65

4.13 Impact of extra ID material on energy scale . . . . . . . . . . . . 66

4.14 Impact of extra ID material on energy scale and fractional energy resolution ...................... . . 6 67

4.15 Nominal longitudinal energy deposition in each calorimeter layer . . . 69

4.16 Fractions of energy deposited in each layer of the electromagnetic barrel calorimeter .................... . . 72

4.17 The estimator $R_{\text {cryo }}$ as a function of $|\eta| \ldots \ldots$. . . . . . . . . . . . . . . . . . . 75

4.18 Longitudinal energy loss profiles for $\mathrm{Pb}, \mathrm{Fe}$ and $\mathrm{LAr}$. . . . . . . . . . 77

4.19 Fraction of energy deposited in each layer of the calorimeter as a function of initial energy . . . . . . . . . . . . . 79

4.20 Sensitivity to extra material of energy deposited in $\mathrm{S} 1$ and $\mathrm{S} 2 \ldots$. . . 82

4.21 The new shower variable, $E_{1} / E_{2} \ldots \ldots$. . . . . . . . 83

4.22 The estimator $R_{\text {cryo }}$ for $E_{1} / E_{2} \ldots \ldots \ldots$. . . . . . . 85

4.23 Statistical significance of the $R_{\text {cryo }}$ estimators . . . . . . . . . . . 86

4.24 Sensitivity of $E_{1} / E_{2}$ to extra cryostat material . . . . . . . . . . . 87

4.25 Results of the 2004 combined test beam . . . . . . . . . . . . 88

4.26 Sensitivity of $E_{1} / E_{2}$ to extra PS-S1 material . . . . . . . . . . . . 90

$4.27 R_{\text {cryo }}$ for the energy deposited in each calorimeter layer with 500,000

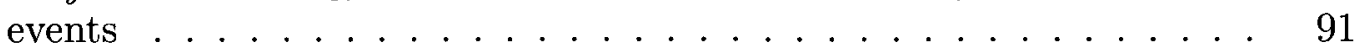

$4.28 R_{\text {cryo }}$ for $E_{1} / E_{2}$ with 500,000 events . . . . . . . . . . . . . 92

4.29 Bremsstrahlung "x-ray" of the inner detector . . . . . . . . . . . . . 94

4.30 Monte Carlo truth $R$ coordinates of bremsstrahlung events . . . . . . 96

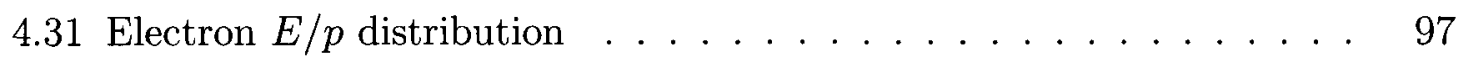

4.32 Fraction of events in the tail of the $E / p$ distribution . . . . . . . . . . 98

$4.33 R_{I D}$ for the energy fraction in each calorimeter layer . . . . . . . . . . 100

$4.34 R_{I D}$ for $E_{1} / E_{2} \ldots \ldots \ldots \ldots \ldots \ldots$

4.35 Average number of bremsstrahlung per event . . . . . . . . . . . 103 
"People are always asking for the latest developments in the unification of this theory with that theory, and they don't give us a chance to tell them anything about what we know pretty well. They always want to know the things we don't know."

Richard Feynman 


\section{Chapter 1}

\section{Introduction}

\subsection{The Standard Model}

Nature can be boiled down to a handful of elementary particles and forces. From J.

5 J. Thomson's discovery of the electron in 1897 using a simple cathode ray tube [1] to the discovery of the top quark in 1995 by large teams of researchers at Fermilab's enormous Tevatron $[2,3]$ to the 2500-person ATLAS collaboration working at the even more enormous Large Hadron Collider, modern particle physicists have devoted over a century to observing and understanding "the substance[s] from which the chemical elements are built up" [1]. 
Our current understanding of matter is based on our observations of elementary particles that can be categorized based on their spins. Two categories of elementary particles exist; these are the fermions, which have half-integer spins, and the bosons, which have integer spins. All matter is made up of these elementary particles. servable properties and the interactions in which they participate; there are three families of each of these two types of fundamental particles. Each of these particles has its own antiparticle, having the same mass and spin but with opposite charge (if charged) and other quantum numbers. The three lepton families consist of the electron $(e)$ and the electron neutrino $\left(\nu_{e}\right)$, the muon $(\mu)$ and the muon neutrino $\left(\nu_{\mu}\right)$ and the tau $(\tau)$ and the tau neutrino $\left(\nu_{\tau}\right)$. Making up the three quark families are the up $(u)$ and down $(d)$ quarks, the charmed $(c)$ and strange $(s)$ quarks, and the top $(t)$ and bottom $(b)$ quarks. Unlike leptons, which are singly-charged or neutral, quarks have fractional electric charges, as well as a so-called "colour" charge. Combinations of two or three quarks and their antiparticles form protons, neutrons and other particles, collectively known as hadrons, which have integer electric charges and are "colourless" by virtue of the colour charges of their consituent quarks cancelling one another out. The hierarchy of quark and lepton masses spans a broad range, from the very nearly massless $\left(m_{\nu}<2 \mathrm{eV}\right.$; note the use of natural units) to the very massive $\left(m_{t}=(171.2 \pm 2.1) \mathrm{GeV}\right)[4]$ 


\begin{tabular}{l||c|r} 
Interaction & Moderator & Relative strength \\
\hline \hline Strong & gluon & 10 \\
\hline Electromagnetic & photon & $10^{-2}$ \\
\hline Weak & $W^{ \pm}, Z$ & $10^{-13}$ \\
\hline Gravity & graviton? & $10^{-42}$
\end{tabular}

TABLE 1.1: The four forces of nature, their moderators and their relative strengths [5].

Every particle interaction can be described by one of four forces; these forces are moderated by bosons, which act as force carriers. The strong interaction, which is responsible for binding quarks together to form hadrons, is moderated by gluons $(g)$. This is the only interaction in which leptons do not participate, as it requires that the interacting particles have colour. Electromagnetism is moderated by the photon $(\gamma)$, and the weak interaction, by which radioactive decay proceeds, is moderated by the $W^{ \pm}$and the $Z$. A fourth interaction, gravity, is thought to be moderated by the graviton, but this particle has not yet been observed experimentally. The four interactions are summarized in Table 1.1.

At an energy scale of about $100 \mathrm{GeV}$, the electromagnetic and weak forces are unified, forming what is known as the electroweak force [6-8]. It is thought that at much higher energies (on the order of $10^{16} \mathrm{GeV}$ ), the electroweak and strong forces are unified, which forms the basis of many so-called Grand Unified Theories [4]. At even higher energies (approximately $10^{19} \mathrm{GeV}$ ), corresponding to the immediate aftermath 
of the Big Bang, it is thought that all four forces are unified.

In an attempt to describe the interactions of the fundamental particles (with the exception of gravity), theorists have developed the Standard Model of particle physics [6-8]. The Standard Model combines the electroweak interaction with the theory of quantum chromodynamics (QCD), which describes the interactions of quarks and gluons. No experiment has managed to refute the postulates of the Standard Model, making it one of the most successful theories in physics to date.

Despite its successes, one prediction of the Standard Model has yet to be confirmed. One major problem, which seems simple on the surface, but which has managed to confound physicists for decades, can be summarized by the question: "Why do some particles have mass, while others are massless?" The answer to this question may lie in a mechanism proposed by Peter Higgs $[9,10]$. The Higgs mechanism, as it has come to be known, describes the manner in which particles acquire mass and accurately predicts the relation between the masses of the $W^{ \pm}$and $Z$ bosons while allowing the photon to remain massless. The Higgs mechanism also predicts the existence of a massive particle known as the Higgs boson, which has yet to be observed.

The Higgs, as it is often called, has proven to be incredibly difficult to detect. Numerous collider experiments have failed to produce any trace of the Higgs since 
it was proposed in 1964. Experiments involving $e^{-} e^{+}$collisions at LEP (the Large

${ }_{65}$ Electron-Positron Collider) have established a lower limit on the Higgs' mass of $m_{H}>$ $114.4 \mathrm{GeV}$ at the $95 \%$ confidence level [11]. Recently, data from $p \bar{p}$ collisions at the Tevatron have excluded the mass range $160 \mathrm{GeV}<m_{H}<170 \mathrm{GeV}$ at the $95 \%$ confidence level [12].

The latest attempt to find the Higgs will soon be underway at the European Large Hadron Collider (LHC), the world's largest particle accelerator, is a protonproton collider scheduled to begin operation in the autumn of 2009 , and will probe the Standard Model at energies never before achieved in the laboratory. At the LHC's incredibly high centre-of-mass energy of $14 \mathrm{TeV}$, the $p p$ collisions will be sufficiently energetic to produce new, heavy particles which will quickly decay into lighter, more stable particles before they can be detected. These decay products, which include such familiar particles as electrons, photons and jets of hadrons, can then be observed experimentally, providing valuable information about the nature of the more exotic particles from which they were produced. Apparatus), a general-purpose detector that will measure the properties of particles produced in the LHC's $p p$ collisions with the goal of finally discovering the Higgs boson, and hopefully even new physics beyond the Standard Model. 


\subsection{Physics Studies at ATLAS}

85

LHC in order to measure a wide variety of Standard Model processes, including the cross section and mass of the top quark and electroweak boson ( $W$ and $Z$ ) cross sections; these measurements would provide precise values for fundamental parameters of the Standard Model. The existence of a Standard Model Higgs will be probed in $H \rightarrow \gamma \gamma, H \rightarrow 4 e$ ), in order to directly observe this unconfirmed prediction of the Standard Model.

Beyond the Standard Model, ATLAS will search for more exotic incarnations of the Higgs, as well as extra dimensions, gravitons and miniature black holes. Supersymmetry (SUSY) studies will involve searches for the decays of such particles as squarks and gluinos (the supersymmetric partners of quarks and gluons; refer to [4] for a concise summary of current SUSY theories) to produce cascades including the lightest supersymmetric particle (LSP). The observation of this particle could confirm the existence of a viable candidate for the weakly interacting, neutral, dark matter that constitutes roughly $22 \%$ of the matter in our universe.

For both the familiar Standard Model and the new and rare processes, excellent identification and high-resolution measurement of leptons, photons and jets of 
hadrons produced from quarks and gluons will be required in order to reconstruct the unstable particles from which they decayed. Since the high QCD background from $p p$ collisions dominates many rare processes, identification of secondary vertices (which indicate the decays of long-lived particles produced in collisions) and missing transverse energy (an imbalance in the amount of energy deposited transverse to the beam line, indicating the presence of a weakly interacting, neutral particle) is also crucial.

\subsection{Motivation for this Work}

ATLAS is composed of multiple subdetectors, including an inner detector, responsible for identifying and tracking charged particles, and an electromagnetic calorimeter system that will measure the energies of electromagnetically interacting particles, namely electrons and photons, that will pass through the detector after being produced in proton-proton collisions. Many of the Standard Model and new physics processes that ATLAS will investigate involve electromagnetically interacting particles as part of the final decay products; for example, we have already seen the case of the Higgs decaying to two photons. Therefore, in order to be able to discover new physics, we must first be able to measure the properties of well-known particles with 
of Standard Model processes, such as the decay $W \rightarrow e \nu$. This process, which is already well understood based on precision measurements at LEP $[14,15]$, is a source of clean, isolated electrons; it provides a useful tool for calibrating the detector so that it will be capable of measuring new physics properties to the highest possible degree of precision.

Of particular importance in the calibration process is the need to develop an accurate model of the material composition and distribution in the inner detector and the calorimeters. When electrons and photons pass through a material, they can initiate cascades of electromagnetically interacting particles; these cascades are also known as electromagnetic showers and can provide useful information about the properties of the incident particle, such as its initial energy. The presence of unexpected extra material in front of the calorimeter can alter the longitudinal development of a shower in the calorimeter and, hence, the measurement of the energy of the incident particle.

The aim of this work is to develop a greater understanding of the response of the electromagnetic barrel calorimeter to extra material in front of this detector. The ATLAS calorimeter is not an isolated machine; it is surrounded by support structures, electronics, cables and other subdetectors. While the current material budget is well documented, the early running period at the LHC will provide the opportunity to fine-tune the understanding of the distribution of material in front 
of the calorimeter and how it affects the calorimeter's response to electromagnetic showers. A method [16] has been developed to probe this response using simulated electrons from $W \rightarrow e \nu$ decays. This method is validated and further developed in this work. The longitudinal development of electromagnetic showers initiated by these electrons in a simulated ATLAS detector, featuring the addition of extra material in several regions, is investigated in order to gauge the sensitivity of the calorimeter to small quantities of extra material.

An introduction to the LHC and ATLAS will be presented in Chapter 2, followed by an in-depth exploration of the theory of electromagnetic showers and calorimetry, as well as an outline of the structure and expected performance of the electromagnetic calorimeter in Chapter 3. Chapter 4 presents the methodology and results of this work. The results of [16] are verified in Section 4.2 and Section 4.3.1; the remainder of Chapter 4 is based on original work. Finally, a summary and conclusions will be given in Chapter 5 . The results presented herein were prepared in the context of this thesis and, as such, are not official results of the ATLAS collaboration. 


\section{Chapter 2}

\section{The LHC and ATLAS}

\subsection{The Large Hadron Collider}

The Large Hadron Collider (LHC) [17, 18], illustrated in Figure 2.1, was constructed in the former LEP tunnel at CERN, approximately $100 \mathrm{~m}$ below the Swiss and French countryside. Measuring $26.7 \mathrm{~km}$ in circumference, the LHC is the largest and most powerful particle accelerator built to date. The LHC is a proton-proton ( $p p)$ collider; at the design energy, two opposing beams of protons will be accelerated until they reach energies of $7 \mathrm{TeV}$ per beam, for a total centre of mass energy of $14 \mathrm{TeV}$.

A proton beam passes through a multi-stage injection chain before reaching its full energy in the LHC ring. First, the Linac2 accelerates the beam to $50 \mathrm{MeV}$. 


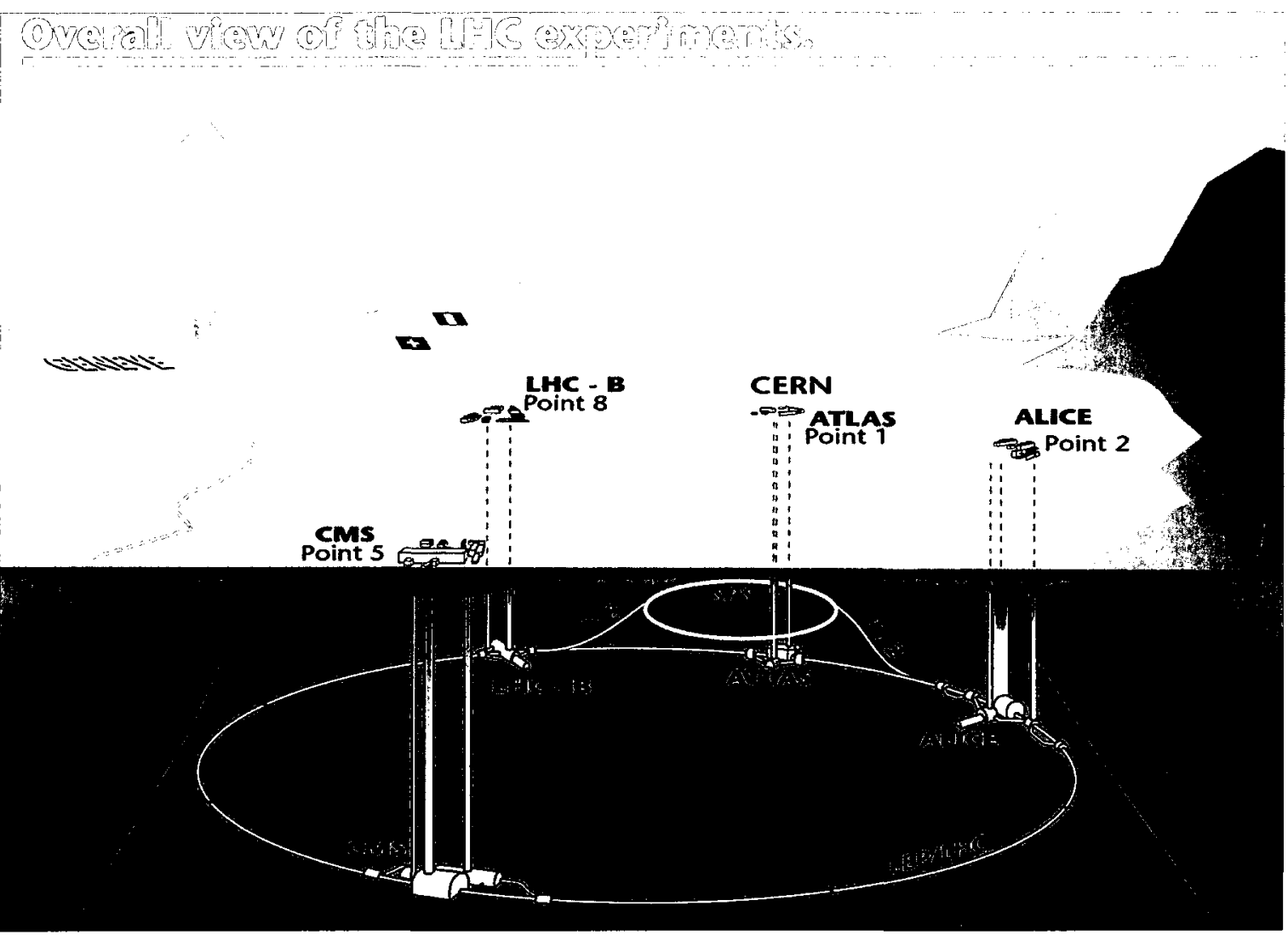

FIGURE 2.1: The LHC ring, showing the positions of the four major experiments: ATLAS, CMS, LHCb and ALICE. Figure from [19].

The beam is then accelerated to $1.4 \mathrm{GeV}$ in the Proton Synchrotron Booster (PSB); it then moves to the Proton Synchrotron (PS). The beam leaves the PS with energy of $25 \mathrm{GeV}$ and enters the Super Proton Synchrotron (SPS), where it is accelerated to $450 \mathrm{GeV}$. This is the last step in the injection chain before the beam enters the LHC ring, where it is accelerated to the design energy of $7 \mathrm{TeV}$.

In the LHC, the beams are contained in two separate beam pipes. They are gradually accelerated until they reach the design energy over a period of 20 minutes 
by a radio frequency (RF) system that boosts the beam energy by $485 \mathrm{keV}$ per turn.

The beams are steered by twin-bore superconducting dipole magnets cooled to below $2 \mathrm{~K}$ by superfluid helium.

The proton beams consist of 2808 bunches of $10^{11}$ protons per bunch; the bunches will cross every $25 \mathrm{~ns}$. At the LHC's design luminosity of $10^{34} \mathrm{~cm}^{-2} \cdot \mathrm{s}^{-1}$, collisions will occur with a frequency of $4 \times 10^{7} \mathrm{~s}^{-1}$. During the 2009-2010 run period, the LHC is expected to operate at a reduced centre of mass energy of $10 \mathrm{TeV}$ and a luminosity of up to $10^{31} \mathrm{~cm}^{-2} \cdot \mathrm{s}^{-1}$, yielding approximately $200 \mathrm{pb}^{-1}$ of data per experiment. After the initial run, the LHC will operate for approximately three years at a luminosity of $10^{33} \mathrm{~cm}^{-2} \cdot \mathrm{s}^{-1}$, yielding $10 \mathrm{fb}^{-1}$ of data per year, followed by an additional three-year period of operating at the full design luminosity, providing 100 $\mathrm{fb}^{-1}$ per year.

The beams collide at four interaction points corresponding to the four major LHC experiments. Two general-purpose experiments, ATLAS (A Toroidal LHC Apparatus) and CMS (Compact Muon Solenoid) study a variety of Standard Model and new physics processes. $\mathrm{LHCb}$ is designed to study the properties of the $b$ quark. The LHC is also capable of colliding lead ions at a luminosity of $10^{27} \mathrm{~cm}^{-2} \cdot \mathrm{s}^{-1}$; ALICE (A Large Ion Collider Experiment) will study these collisions. This work concerns the ATLAS experiment. 


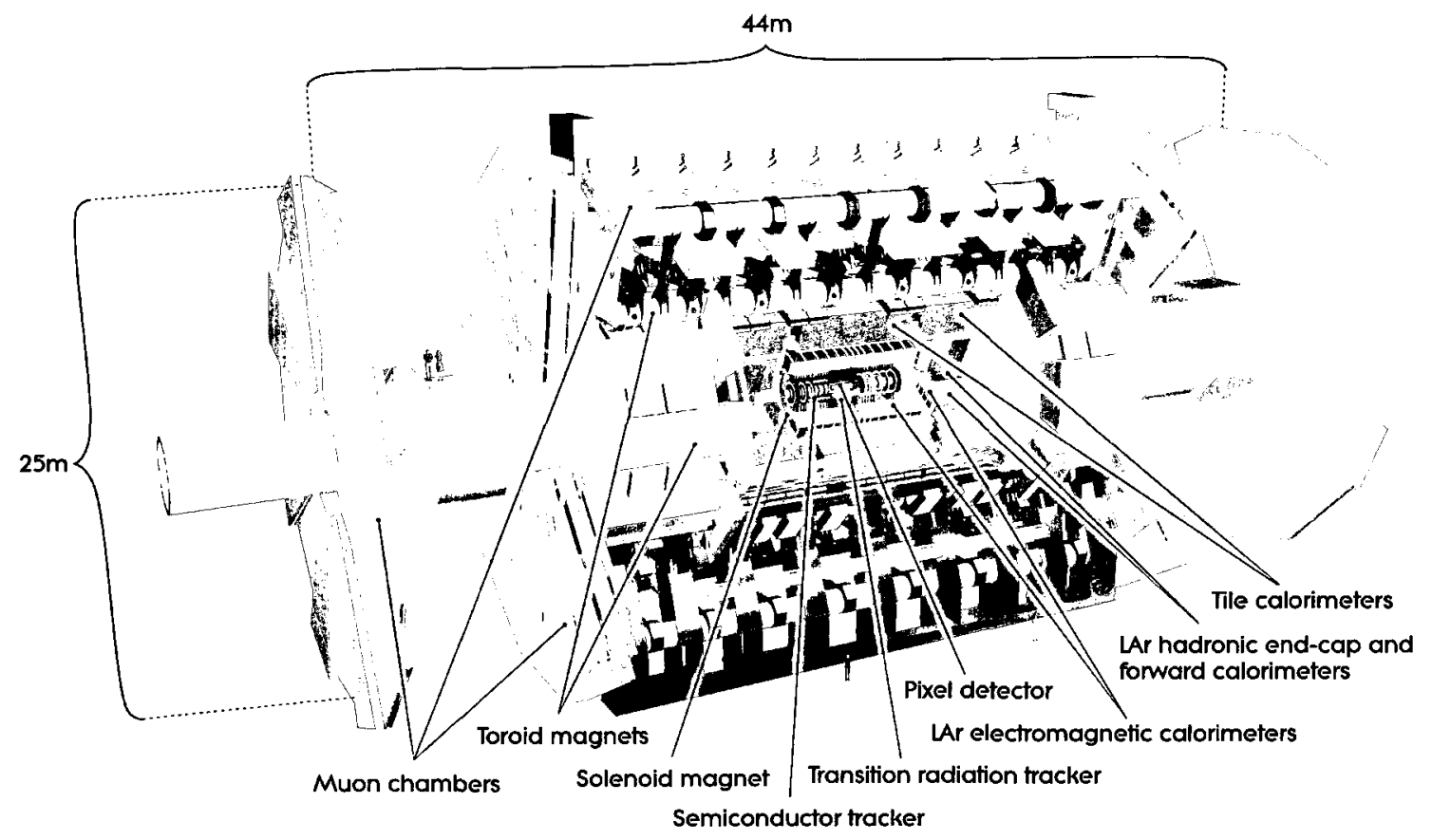

Figure 2.2: Cutaway view of ATLAS, showing the subdetector systems. Figure from [13].

\subsection{ATLAS}

The wide-ranging physics program at ATLAS [13] has been designed to take advantage

of the high luminosity and increased physics cross sections at the LHC in order to perform high-precision measurements of Standard Model properties and to search for rare and new physics processes.

ATLAS, pictured in Figure 2.2, consists of concentric layers of subdetectors, which measure the properties and interactions of the wide variety of particles produced during $p p$ collisions. At the design luminosity, approximately 23 inelastic collisions 
will occur on average at every bunch crossing, based on an inelastic cross section of $80 \mathrm{mb}$. This results in approximately 1000 charged particle tracks traversing the inner detector and approximately $1400 \mathrm{GeV}$ of transverse energy being deposited in the calorimeters every $25 \mathrm{~ns}$. The unprecedented high luminosity at the LHC necessitates the use of fast, radiation-hard detectors and electronics in order to withstand the high flux of radiation and minimize pileup noise caused by overlapping signals from adjacent bunch crossings.

The following briefly describes each of the subdetectors and their functions in the overall detection scheme.

\subsubsection{ATLAS Coordinate System}

The detector is nominally forward-backward symmetric about the interaction point, which is located in at the centre of the detector; this point defines the origin of the coordinate system. The positive $x$-axis points inwards from the interaction point to the centre of the LHC ring and the positive $y$-axis points upwards. The $z$-axis lies in the direction of the beam line; the side of the detector with $z<0$ is known as side $\mathrm{C}$ and the side having $z>0$ is known as side A. The azimuthal angle, $\phi$, is measured around the $z$-axis, while the polar angle, $\theta$, is measured from the $z$-axis. Instead of 
the polar angle, the pseudorapidity $\eta$, defined as

$$
\eta=-\ln \left[\tan \left(\frac{\theta}{2}\right)\right]
$$

is often used. Hence, the distance in $\eta-\phi$ space, $\Delta R$, is given by

$$
\Delta R=\sqrt{\Delta \eta^{2}+\Delta \phi^{2}}
$$

The transverse momentum $p_{T}$, transverse energy $E_{T}$ and the missing transverse energy $E_{T}^{m i s s}$ are measured in the $x-y$ plane, which lies transverse to the direction of the beams.

\subsubsection{Inner Detector}

The inner detector $[13,20,21]$, shown in Figure 2.3, is responsible for measurements of momentum and primary and secondary vertices, as well as pattern recognition and electron identification. It tracks charged particles having $p_{T}>0.5 \mathrm{GeV}$. Located in the area immediately surrounding the beam pipe, the inner detector is immersed in a $2 \mathrm{~T}$ solenoidal field, which serves to bend the paths of charged particles. The inner detector, which covers the region $|\eta|<2.5$, measures $6.2 \mathrm{~m}$ in length and has an outer 


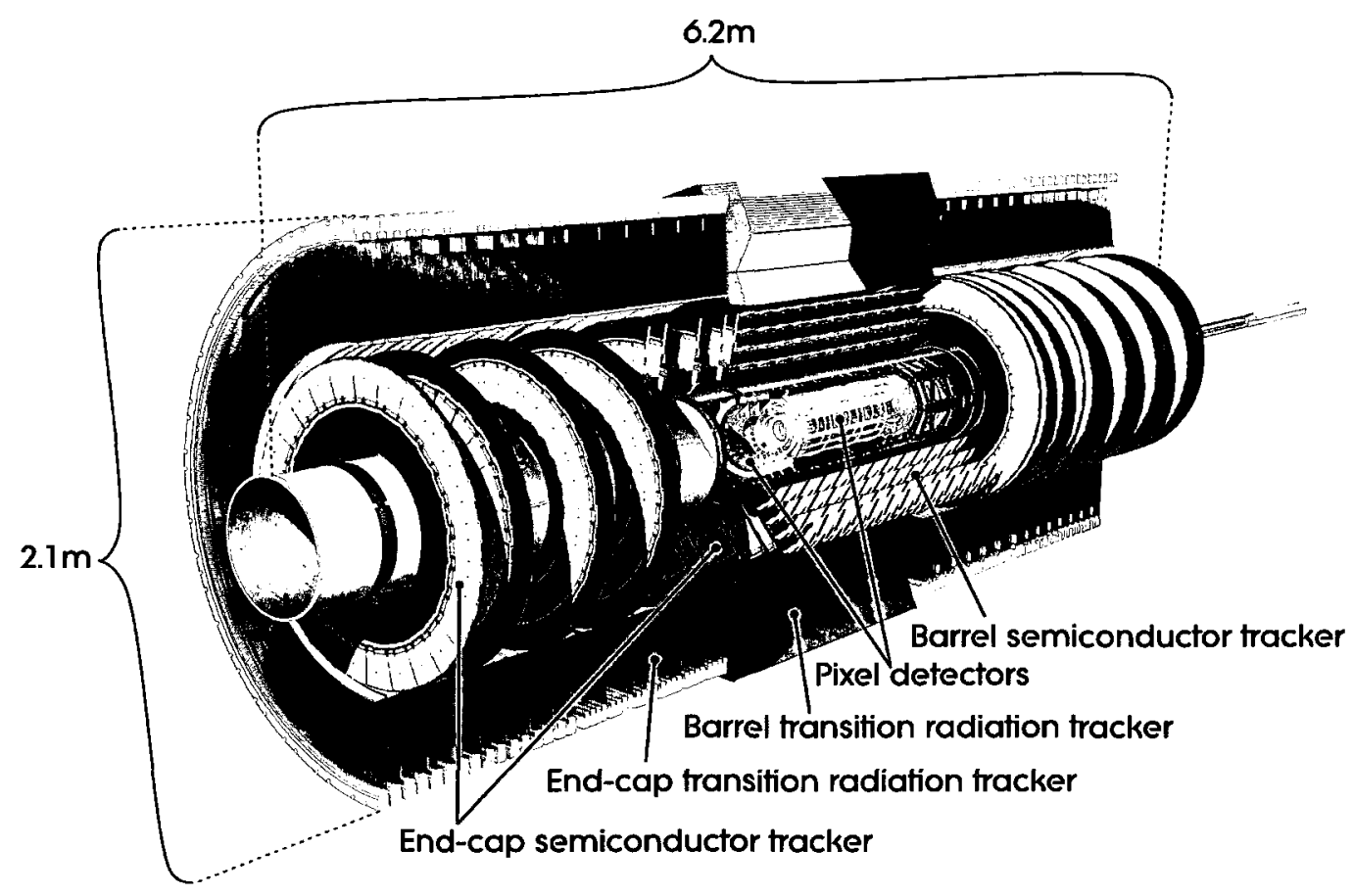

Figure 2.3: Cutaway view of the inner detector. Figure from [13].

230 $50 \mathrm{~mm}$ from the beam axis in order to achieve accurate vertex reconstruction.

The inner detector consists of three separate subsystems which, when combined, provide excellent tracking performance, with $p_{T}$ resolution given by $\sigma_{p_{T}} / p_{T}=$ $0.05 \% \cdot p_{T} \oplus 1 \%$, where $\sigma_{p_{T}}$ is the spread of the transverse momentum measurements and $p_{T}$ is measured in $\mathrm{GeV}$. The innermost subsystem consists of three layers of silicon pixels laid out in concentric cylinders in the barrel or on disks perpendicular to the beam axis in the endcaps. The second subsystem, the semiconductor tracker (SCT), 
consists of four layers of silicon microstrips in the barrel and nine disks in each endcap. Together, the SCT, with its 6.3 million readout channels, and the pixels, with resolution and electron identification.

\subsubsection{Calorimeters}

The ATLAS calorimetry system $[13,22,23]$, shown in Figure 2.4, consists of two subsystems designed to measure the energies of electromagnetic and hadronic showers. All of the ATLAS calorimeters are sampling calorimeters; this particular type of calorimeter is discussed in detail in Section 3.2. The calorimeters must be able to measure energies from the $\mathrm{MeV}$ scale up to the $\mathrm{TeV}$ scale while still providing high precision (the energy scale in the electromagnetic calorimeter must be measured to $0.02 \%$ for $W$ mass measurements). In order to provide full shower containment, the electromagnetic (hadronic) calorimeter has a minimum thickness of 22 radiation lengths (9.7 interaction lengths). High granularity allows for high-precision energy 


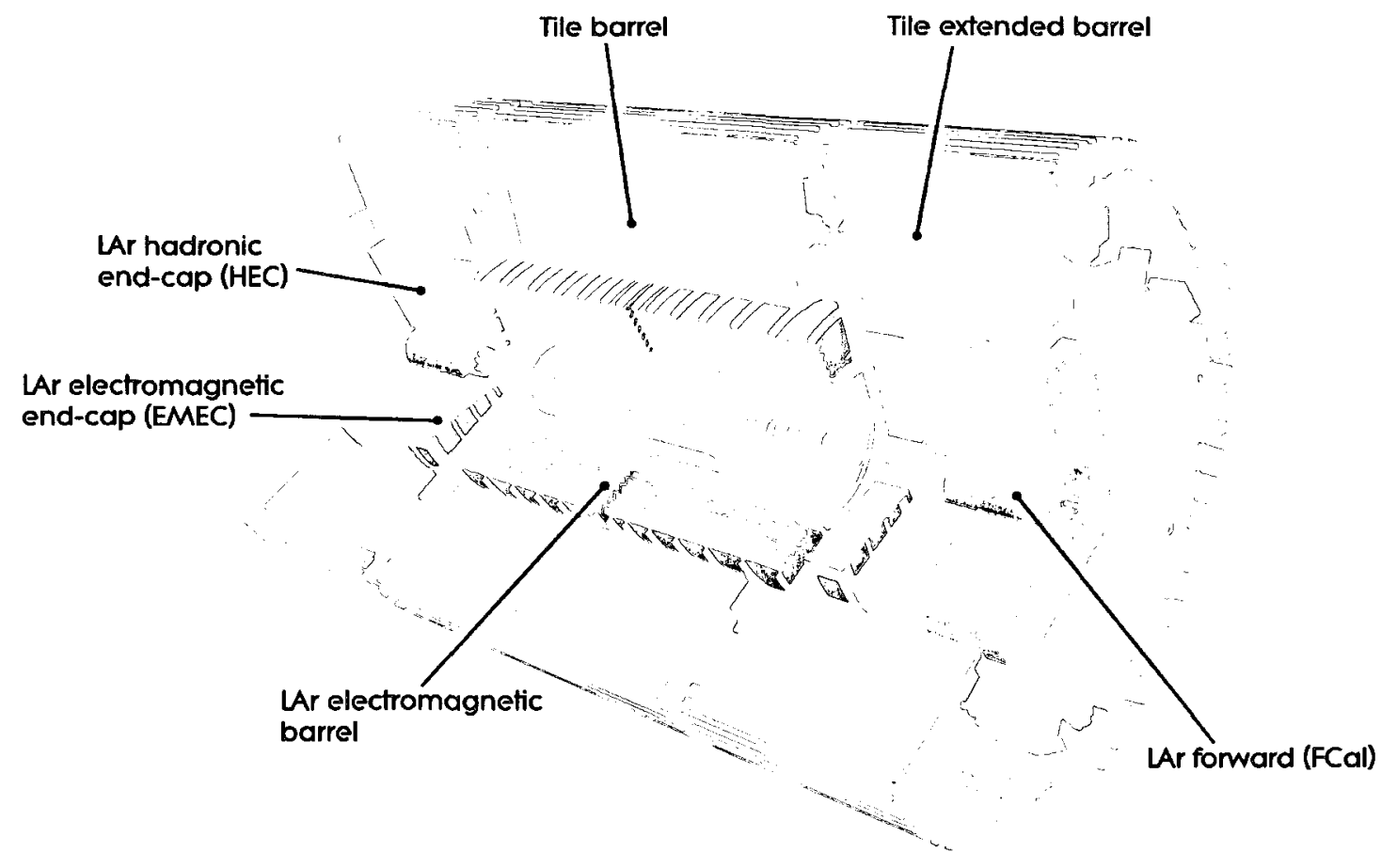

FIgure 2.4: Cutaway view of the calorimeters. Figure from [13].

and position measurements, and large pseudorapidity coverage $(|\eta|<4.9)$ is important for missing energy measurements.

The electromagnetic calorimeter, described in detail in Section 3.4, is a leadliquid argon sampling calorimeter with a custom-designed accordion geometry. The electromagnetic calorimeter is divided into a barrel section (EMB) and two endcap (EMEC) sections. In the region in $|\eta|$ corresponding to the inner detector, the granularity is very fine in order to provide precision measurements of electrons and photons. The required energy resolution is on the order of $\sigma_{E} / E=10 \% / \sqrt{E} \oplus 0.7 \%$, where $\sigma_{E}$ 
is the spread of the energy measurements and $E$ is measured in $\mathrm{GeV}$.

The hadronic calorimetry system is broken down into several overlapping subsystems. In the barrel, the tile calorimeter covers the region $|\eta|<1.0$ and measures $5.8 \mathrm{~m}$ in length. The tile calorimeter also has two extended barrels, each measuring $2.6 \mathrm{~m}$ long and covering the region $0.8<|\eta|<1$.7. Steel functions as the absorber medium for this subsystem, while the active sampling medium consists of scintillating tiles. Readout is performed via wavelength-shifting fibres, which relay the signal to photomultiplier tubes. In the barrel and the endcap, the required energy resolution is $\sigma_{E} / E=50 \% / \sqrt{E} \oplus 3 \%$, where $E$ is in $\mathrm{GeV}$.

In addition to the tile calorimeter, the hadronic calorimetry system includes 275 two liquid argon calorimeter subsystems. The hadronic endcap calorimeter (HEC), which uses copper as the absorber, consists of two wheels per endcap and is located behind the EMEC. The HEC provides coverage in the region $1.5<|\eta|<3.2$. Also located in the endcap cryostats are the forward calorimeters (FCal), which cover the region $3.1<|\eta|<4.9$. The FCal consists of three separate modules per endcap; the first module is actually a copper-liquid argon electromagnetic calorimeter, while the second and third modules are hadronic calorimeters having tungsten as the absorber medium. All of the FCal modules follow the same basic design: each consists of a metal matrix having evenly-spaced longitudinal channels filled with electrodes in the form of rods and tubes, between which liquid argon gaps are positioned. The energy 
resolution of the FCal must be on the order of $\sigma_{E} / E=100 \% / \sqrt{E} \oplus 10 \%$, where $E$ is in units of $\mathrm{GeV}$.

\subsubsection{Muon Spectrometer}

The muon spectrometer $[13,24]$, shown in Figure 2.5, is designed to detect and measure the momentum of charged particles (i.e. mainly muons) leaving the calorimeter system in the region $|\eta|<2.7$ and to trigger on these particles in the region $|\eta|<2.4$. For muons with transverse momentum of about $1 \mathrm{TeV}$, the resolution must be on the order of $\sigma_{p_{T}} / p_{T}=10 \%$. The system relies on the deflection of muon tracks by three superconducting air-core toroid magnets: one in the barrel region $(|\eta|<1.4)$ and two in the endcap regions $(1.6<|\eta|<2.7)$. In the transition region, $1.4<|\eta|<1.6$, deflection is accomplished by both the barrel and the endcap toroid magnets.

In the barrel, the muon tracking chambers form three concentric cylindrical layers located at $5 \mathrm{~m}, 7.5 \mathrm{~m}$ and $10 \mathrm{~m}$ from the interaction point. In the endcaps, the muon chambers are arranged in four wheels located at $7.4 \mathrm{~m}, 10.8 \mathrm{~m}, 14 \mathrm{~m}$ and $21.5 \mathrm{~m}$ from the interaction point and oriented perpendicular to the beam axis.

Four subdetector systems make up the muon spectrometer. The monitored drift tubes perform precision tracking measurements. They cover the region $|\eta|<2.7$, except in the innermost layer, where cathode strip chambers are instead used in the 


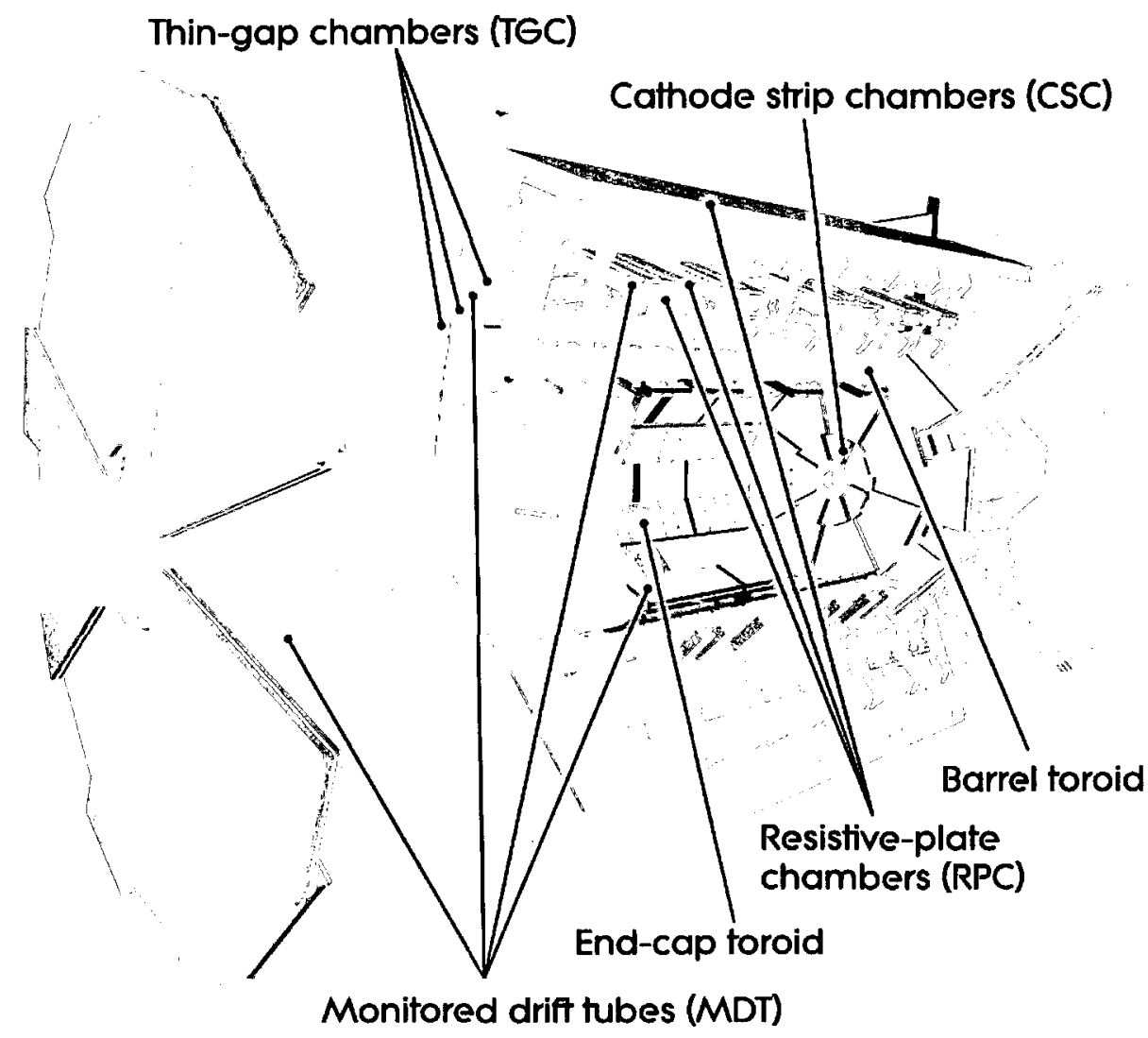

Figure 2.5: Cutaway view of the muon system. Figure from [13].

region $2.0<|\eta|<2.7$. Muon triggering occurs in the resistive plate chambers, which cover the region $|\eta|<1.05$ and in the thin gap chambers, which cover the region $1.05<|\eta|<2.7$

\subsubsection{Trigger}

The trigger system $[13,25,26]$ is responsible for the formidable task of reducing the event data-taking rate from $1 \mathrm{GHz}$ (the proton-proton interaction rate at the design 
luminosity of $10^{34} \mathrm{~cm}^{-2} \cdot \mathrm{s}^{-1}$ ) to a more manageable $200 \mathrm{~Hz}$. This feat is accomplished in three stages corresponding to three trigger levels: the Level-1 (L1) and Level-2 (L2) trigger systems and the event filter.

The hardware-based L1 trigger reduces the data rate to approximately $75 \mathrm{kHz}$. In less than $2.5 \mu \mathrm{s}$, it selects events based on the production of high $p_{T}$ muons, electrons, photons, jets and $\tau$ leptons that decay into hadrons, as well as large $E_{T}$ and $E_{T}^{\text {miss }}$, based on information from the muon spectrometer and the calorimeters. The L1 trigger also defines regions of interest - locations in $\eta$ and $\phi$ containing particularly interesting features; for example, in the calorimeter, a region of interest would be defined as an area of $\Delta \eta \times \Delta \phi=0.1 \times 0.1$ in which a large amount of energy has been deposited. Events that pass the L1 trigger cuts are then passed to the L2 trigger and then the event filter, which together make up the High-Level Trigger (HLT), which is a software-based system.

The L2 trigger makes use of all available information within the regions of interest identified by the L1 trigger, as well as information from the inner detector. It relies on combinations of trigger cuts, known as menus, to process events in $40 \mathrm{~ms}$ and reduce the data rate to roughly $3.5 \mathrm{kHz}$. Finally, events passing the $\mathrm{L} 2$ cuts are transferred to the event filter. Events selected by this final trigger level are classified into predefined data streams corresponding to electrons, muons, jets, photons, $E_{T}^{\text {miss }}$, $\tau$-leptons and $B$-physics. 


\section{Chapter 3}

\section{so The ATLAS Electromagnetic}

\section{Calorimeter}

Electromagnetically interacting particles such as electrons and photons will play an important role in ATLAS, in both calibration and precision measurements of Standard Model processes (e.g. $W \rightarrow e \nu$ ) and in searches for new particles (e.g. $H \rightarrow \gamma \gamma$

and $H \rightarrow 4 e$ ). Electrons and photons lose energy through a variety of mechanisms, but at high energies, the dominant processes are bremsstrahlung (for electrons) and pair production (for photons), which lead to the formation and propagation of electromagnetic showers in a material. The energy deposited over the course of an electromagnetic shower can be measured by a device known as a calorimeter. After a 
brief survey of electromagnetic shower theory, we will introduce the principles of electromagnetic calorimetry. Finally, we will outline the performance requirements and design specifications of the ATLAS electromagnetic calorimeter.

\subsection{Electromagnetic Showers}

\subsubsection{Energy Loss of Electrons and Photons}




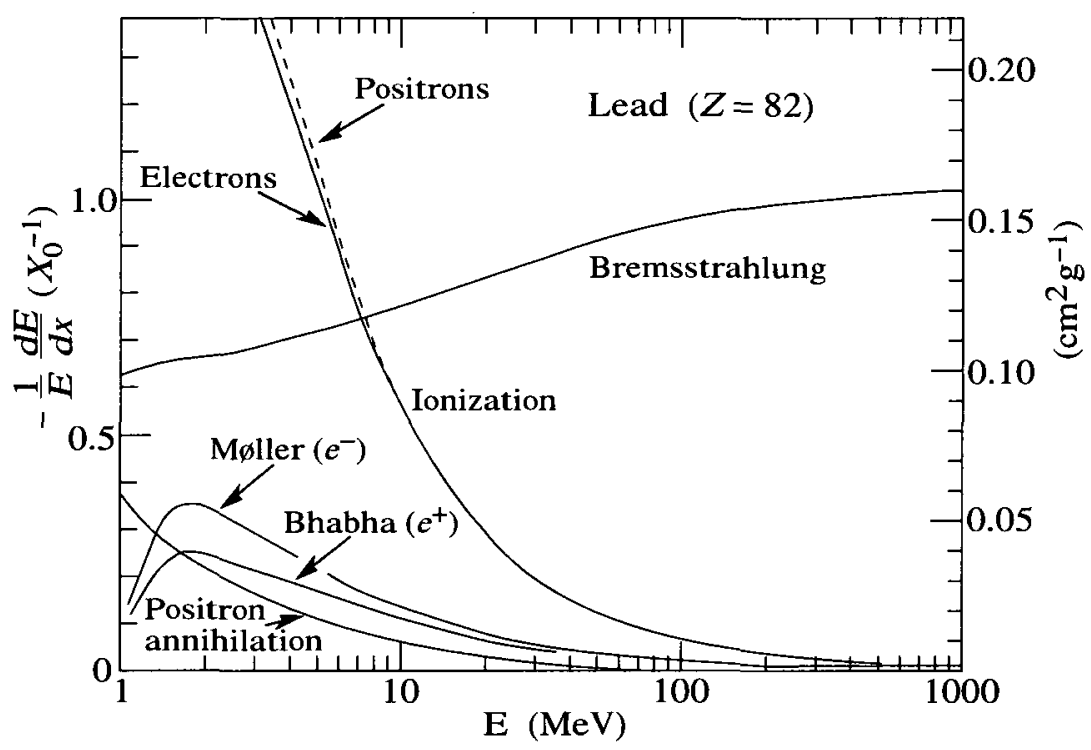

Figure 3.1: Energy loss of electrons in lead. At energies higher than a few tens of $\mathrm{MeV}$, bremsstrahlung dominates. Figure from [4].

In the case of photons passing through a material, there are three main ways by which energy may be lost: the photoelectric effect, Compton scattering, and pair production [27], as illustrated in Figure 3.2. In the first case, a photon having kinetic energy greater than the binding energy of an atomic electron is absorbed, resulting in the ejection of a so-called photoelectron with energy equal to the difference between the kinetic energy of the incident photon and the binding energy of the atomic electron. In the Compton effect, an incident photon scatters from an electron. The electron recoils, and the scattered photon's wavelength is lower than that of the incident photon. In the case of pair production, an intense electric field causes a photon to decay to an electron-positron pair. It may occur near a nucleus or an atomic electron; in the former case, the threshold energy is $2 m c^{2}$, while in the latter case, 


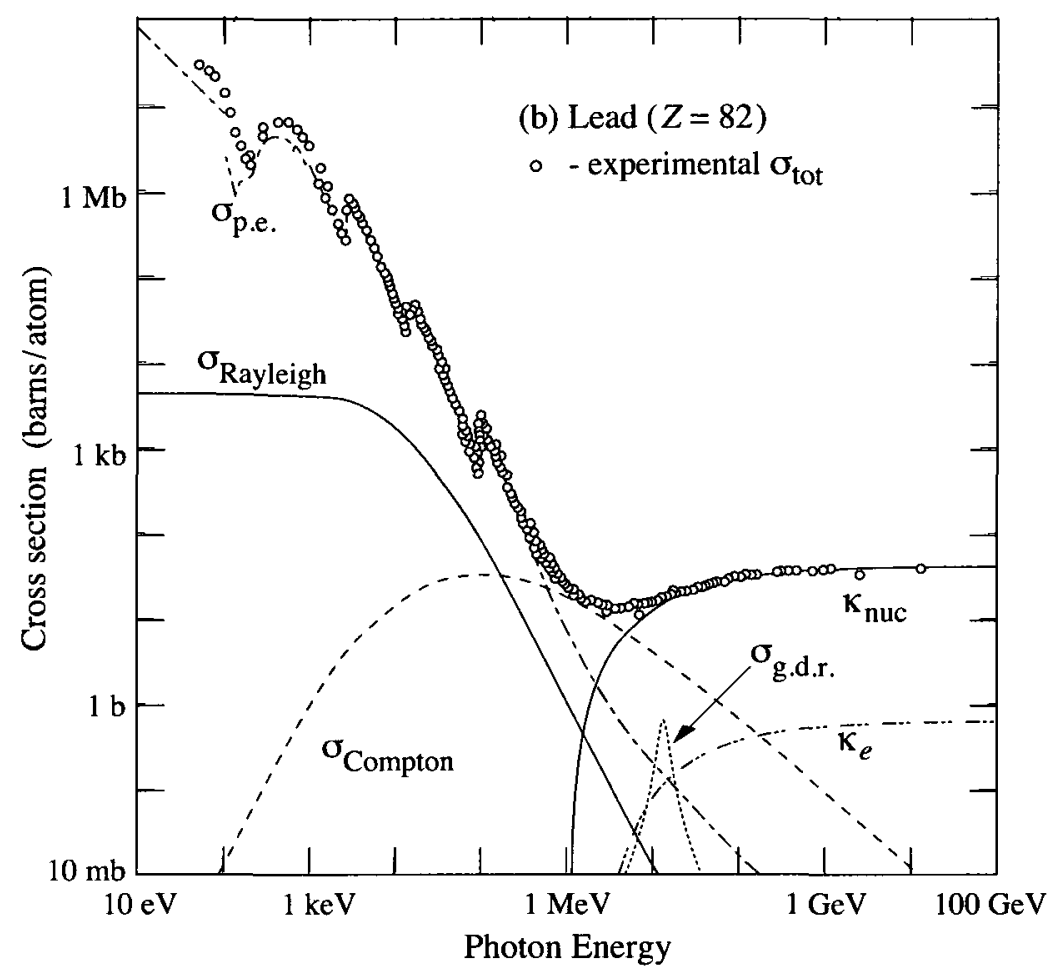

Figure 3.2: Photon total cross sections as a function of energy in lead. At energy higher than $100 \mathrm{MeV}$, pair production $(\kappa)$ dominates. Figure from [4].

the threshold energy is $4 m c^{2}$. Pair production dominates at energies greater than $100 \mathrm{MeV}$.

The energy range of the primary electrons studied in this work has a lower 370 limit of $15 \mathrm{GeV}$, well above the thresholds for the dominance of bremsstrahlung and pair production. Hence, only these processes will be considered. 


\subsubsection{Longitudinal Development of Electromagnetic Showers}

The average distance travelled in matter by an electron before it emits a photon is known as the radiation length $X_{0}$. This quantity is a characteristic of the material

375 1/e (where $e$ represents Euler's number) via radiation [29]. For photons, one radiation length is $7 / 9$ of the mean free path for pair production. For a given material, the radiation length may be approximated by [4]

$$
X_{0}=\frac{1}{\rho} \frac{716.4 \mathrm{~g} \cdot \mathrm{cm}^{-2} A}{Z(Z+1) \ln (287 / \sqrt{Z})}[\mathrm{cm}]
$$

where $\rho$ is the density of the material in $\mathrm{g} \cdot \mathrm{cm}^{-3}, Z$ is the atomic number and $A$ is the atomic weight. Multiplying this by the density will result in a quantity measured in $\mathrm{g} \cdot \mathrm{cm}^{-2}$; this unit is often used instead of $\mathrm{cm}$. Radiation lengths for common materials have been tabulated; for example, the radiation lengths of lead and liquid argon,

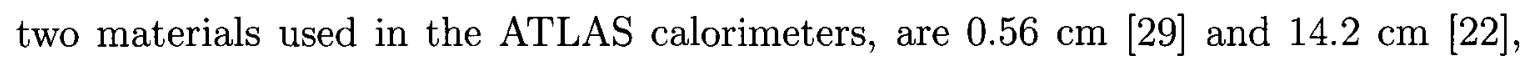
respectively.

When a high-energy electron or photon passes through a material, an electromagnetic shower may occur. Although the generation of particles in an electromagnetic shower is a statistical process, we can explore the development of a shower with 
the following simplified example (illustrated in Figure 3.3), starting with a single electron. After travelling one radiation length, on average, the electron will emit a photon via bremsstrahlung. This photon will then travel on average slightly more than one radiation length before undergoing pair production, resulting in an electron-positron pair in addition to the original electron, which will emit another photon after travelling an additional radiation length. Thus, at a shower depth of approximately two radiation lengths, we will have, on average, four particles: two electrons, one positron and one photon. In other words, at a depth of $t$ radiation lengths, the average number of particles present is given by

$$
N(t) \approx 2^{t} .
$$

In terms of the initial energy $E_{0}$, the average energy of each shower particle at a depth $t$ is [29]

$$
E(t) \approx \frac{E_{0}}{2^{t}}
$$

An electromagnetic shower will cease to develop when the energy of the parti400 cles is equal to the critical energy; at this point, the energy lost via radiative processes will be equal to the energy lost via ionization. The critical energy is specific to the material in which the shower takes place, and it may be calculated for solids and liquids using the formula [4]

$$
E_{c}=\frac{610 \mathrm{MeV}}{Z+1.24} .
$$




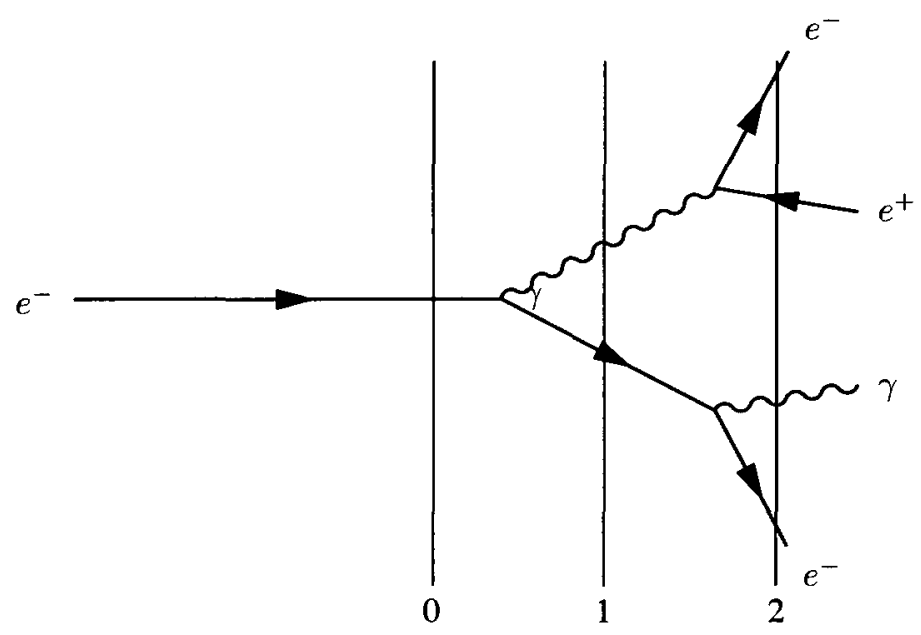

Figure 3.3: An electron-initiated electromagnetic shower. After two radiation lengths, on average, four particles are present.

The critical energies of lead and liquid argon are $9.51 \mathrm{MeV}[29]$ and $30.5 \mathrm{MeV}$ [22], respectively. The maximum number of particles in a shower will be present at the critical energy; they will be found at the depth [29]

$$
t_{m a x}=\frac{\ln \left(E_{0} / E_{c}\right)}{\ln 2}
$$

Hence, the maximum number of shower particles is given by

$$
N_{\max }=e^{t_{\max } \ln 2}=\frac{E_{0}}{E_{c}}
$$

The previous example uses a simplified model to paint a general picture of the development of electromagnetic showers. Empirically, the longitudinal profile of 
energy loss in an electromagnetic shower is given by [4]

$$
\frac{d E}{d t}=E_{0} b \frac{(b t)^{a-1} e^{-b t}}{\Gamma(a)}
$$

where $a$ and $b$ are material-dependent parameters and $\Gamma$ is the gamma function. The value of $b$ has been tabulated for some common materials [4]; it is generally safe to assume an approximate value of $b \approx 0.5$. Once the value of $b$ has been obtained, the value of $a$ may then be calculated using the empirical relation for the maximum shower depth

$$
t_{\max }=\frac{a-1}{b}=\ln y+C_{i}
$$

where $y=E_{0} / E_{c}$ and $C_{i}$ takes a value of either -0.5 in the case of electron-induced showers or +0.5 in the case of photon-induced showers. A longitudinal energy loss profile for a $35 \mathrm{GeV}$ electron traversing $50 X_{0}$ of lead, obtained using Equation 3.7, is shown in Figure 3.4.

\subsubsection{Transverse Development of Electromagnetic Showers}

The transverse progression of an electromagnetic shower is characterized by the Molière radius, given by [29]

$$
R_{M}=X_{0} \frac{21.2 \mathrm{MeV}}{E_{c}}[\mathrm{~cm}]
$$




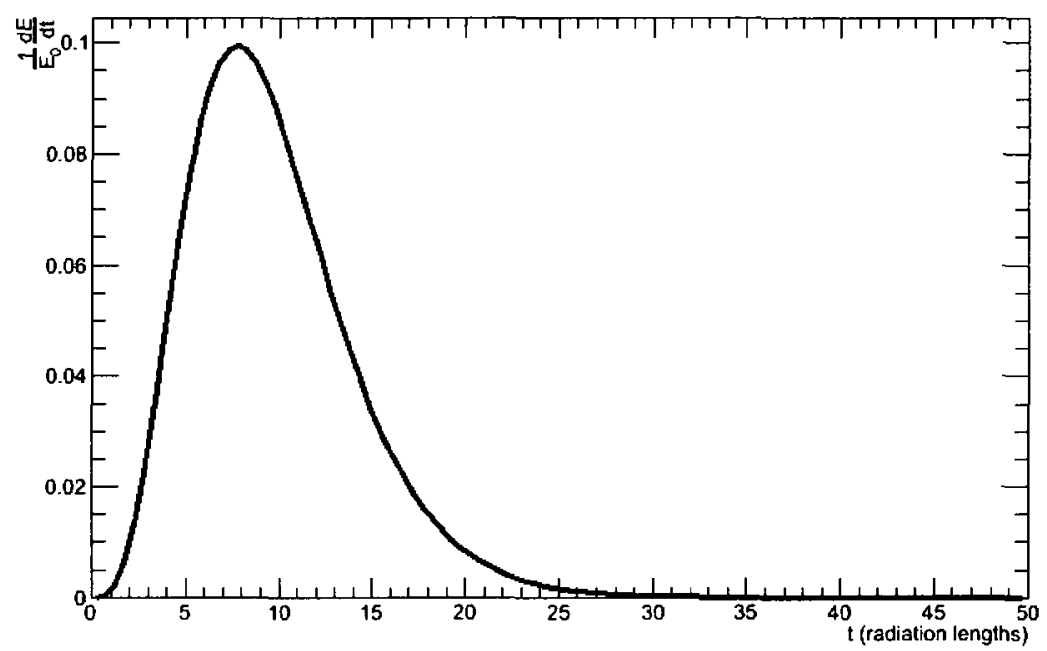

Figure 3.4: Longitudinal energy loss profile of a $35 \mathrm{GeV}$ electron in lead. The maximum number of particles will be present at the depth $t_{\max }=7.71 X_{0}$.

Approximately $90 \%$ of the shower is contained within $2 R_{M}$ of the longitudinal axis. At high energy, shower particles are emitted at small angles, and so the majority of the shower development occurs in the direction of the original particle.

\subsection{Introduction to Electromagnetic Calorimetry}

A calorimeter is a device that measures the energy deposited by particles traversing its active medium. Calorimeters are particularly useful since they provide the only method for detecting neutral particles, such as photons. ATLAS contains two types 
electrons, positrons and photons, while the other detects showers of hadrons. For the purpose of this work, we will focus on electromagnetic calorimeters.

There are two types of electromagnetic calorimeters. The first type, homogeneous calorimeters, consist of a single material and measure the deposited energy continuously. The second type, sampling calorimeters, measure energy at periodic intervals [27]. A typical sampling calorimeter is made up of alternating layers of an absorber material (typically a metal radiator), which serve to generate the shower, and an active medium, which samples the energy of the shower. Given that the metal layers serve to enhance the development of the shower, a very dense metal, such as lead, is used. The sampling layers can consist of a solid or liquid scintillator, a gas, or a liquid noble element. The ATLAS electromagnetic calorimeter is a sampling calorimeter consisting of alternating layers of lead and liquid argon.

In a liquid argon sampling calorimeter, a high voltage is applied across the liquid argon-filled gaps. As the electrons produced in showers pass through the liquid argon, the argon is ionized, yielding electrons which then drift to the centre of the gaps, where the resulting current is read out. The total ionization signal is proportional to the energy of the shower. 
The energy resolution of an electromagnetic sampling calorimeter is parametrized by [4]

$$
\frac{\sigma_{E}}{E}=\frac{a}{\sqrt{E}} \oplus b \oplus \frac{c}{E},
$$

450

fluctuations of the shower, the constant term, $b$, depends on mechanical defects and calibration errors, as well as energy leakage in the event of incomplete shower containment, and $c$ is proportional to the electronic noise.

\subsection{Performance Requirements}

The LHC's unprecedented high energy and luminosity, outlined in Section 2.1, necessitate the use of a custom-designed, state-of-the-art electromagnetic calorimetry system in ATLAS. The electromagnetic calorimeter must be able to reconstruct electrons and photons in the energy range of $5 \mathrm{GeV}$ to $5 \mathrm{TeV}$ [30]. Fast, high-performance sampling components and electronics are required in order to minimize electronic and pileup (superposition of signals from the same or multiple bunch crossings) noise. The detector components must be able to withstand large doses of radiation on the order of $1.2 \mathrm{kGy}-150 \mathrm{kGy}$, depending on their location, over approximately seven years [13]. 
The ATLAS electromagnetic calorimeter will play an important role in the

\subsection{Electromagnetic Calorimeter Design}

The ATLAS electromagnetic calorimeter is a lead-liquid argon sampling calorimeter, located in the area surrounding the inner detector. It is subdivided into three main sections, each of which is located in its own cryostat. Two endcaps cover the region 
$1.375<|\eta|<3.2$, while the barrel (actually two half-barrels separated by a $4 \mathrm{~mm}$ gap at $z=0$ ) covers the region $|\eta|<1.475$ [13]. The front face of the barrel (endcap) calorimeter is located at a radial distance of $1.50 \mathrm{~m}(0.33 \mathrm{~m})$ from the beam line [22].

In order for the electromagnetic calorimeter to make accurate energy and missing energy measurements, it must be able to fully contain electromagnetic showers. For this reason, the calorimeter is at least $22 X_{0}$ thick in the barrel region and each endcap is at least $24 X_{0}$ thick [13]. This motivated the choice of lead, which has a relatively short radiation length, as the absorber material. Liquid argon was chosen as the active sampling material due to its intrinsic stability and linear response, its radiation-hardness and its inability to capture free electrons.

A novel geometry has been designed for the electromagnetic calorimeter. The absorbers and readout electrodes have an accordion shape; this unique geometry permits the detector to be completely symmetrical in $\phi$. In the endcap, the accordion waves run axially, while in the barrel, the waves run in $\phi$; in both regions the folding angles vary with the radius in order to maintain a constant thickness for the sampling region. The accordion has three active layers in the precision region $(|\eta|<2.5)$ and two in the region $2.5<|\eta|<3.2$ and in the region where the barrel and the endcap overlap. The first layer is finely segmented in $\eta$ to provide precise position measurements. Photon direction measurements are obtained from the locations of clusters in the first and second layers. 


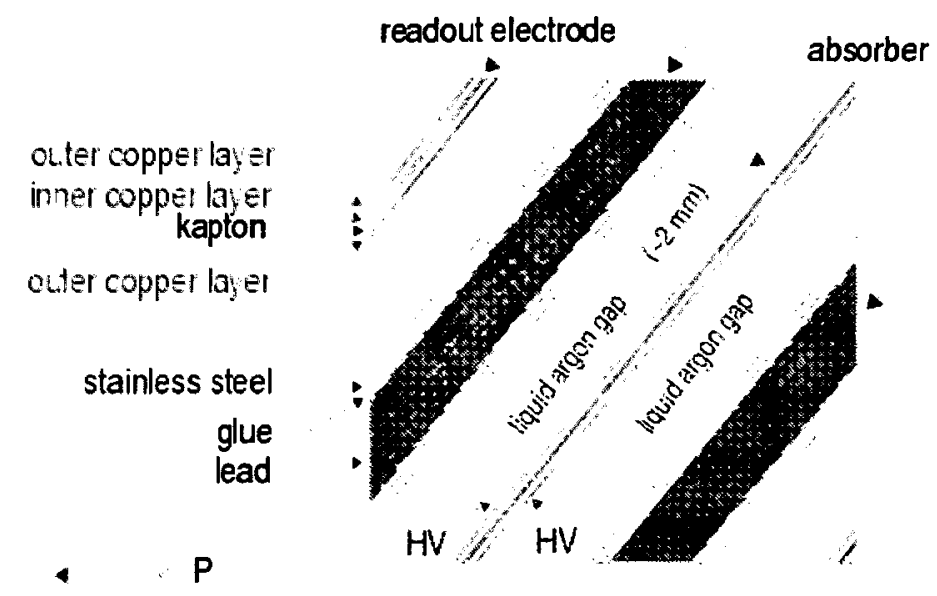

Figure 3.5: Close-up schematic of the absorbers, showing the location of the readout electrodes in the liquid argon gaps. Figure from [31].

The absorbers consist of lead plates sandwiched between two stainless steel sheets, attached with resin-impregnated fibreglass. In the barrel, the lead plates have a thickness of $1.53 \mathrm{~mm}(|\eta|<0.8)$ or $1.13 \mathrm{~mm}(|\eta|>0.8)$, while in the endcap, they measure $1.7 \mathrm{~mm}(|\eta|<2.5)$ or $2.2 \mathrm{~mm}(|\eta|>2.5)$. The readout electrodes, illustrated in Figure 3.5, are located in the gaps between the absorbers and consist of three layers of copper, separated by insulating polyimide (Kapton) sheets. The two outer copper layers are maintained at a potential of $+2000 \mathrm{~V}$, while the inner layer reads out the signal by capacitive coupling. The drift gap on either side of the electrodes measures $2.1 \mathrm{~mm}$, resulting in a $450 \mathrm{~ns}$ drift time at the operating voltage. In order to facilitate the assembly of the calorimeter, two different types of electrodes are used in the barrel, with the boundary between the two types located at $|\eta|=0.8[32]$. Similarly, different types of electrodes are used in the inner and outer 

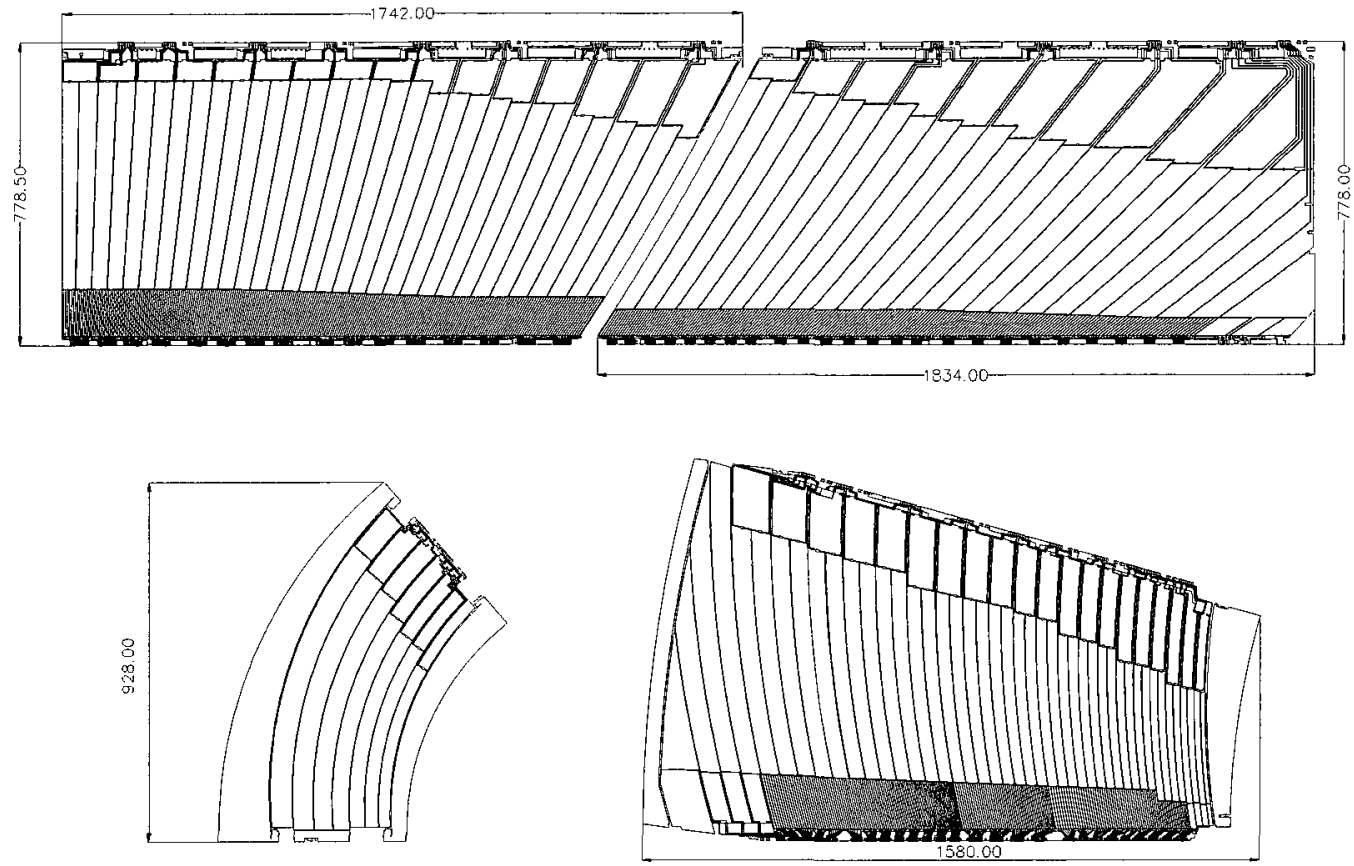

FIGURE 3.6: Four types of electrodes are used in the electromagnetic calorimeter. The top left (right) style is used in the barrel at $|\eta|<0.8(|\eta|>0.8)$. The bottom left (right) style is used in the inner (outer) endcap wheel. Dimensions are given in $\mathrm{mm}$. Figure from [13].

endcap wheels. Figure 3.6 shows the four electrode designs. Multiple electrodes are grouped together in readout cells.

The accordion is divided into modules, each of which consists of 64 lead absorbers and 3424 readout cells. A module is shown in Figure 3.7. A total of 16 of these modules, each spanning $\Delta \phi=22.5^{\circ}$, form a half-barrel; the accordion geometry eliminates the problem of discontinuities between the modules (with the exception of a $4 \mathrm{~mm}$ gap between the half-barrels at $z=0$ ). Each half-barrel measures $3.2 \mathrm{~m}$ long, weighs 57 tonnes and has an inner (outer) diameter of $2.8 \mathrm{~m}(4 \mathrm{~m})$ [13]. The barrel 


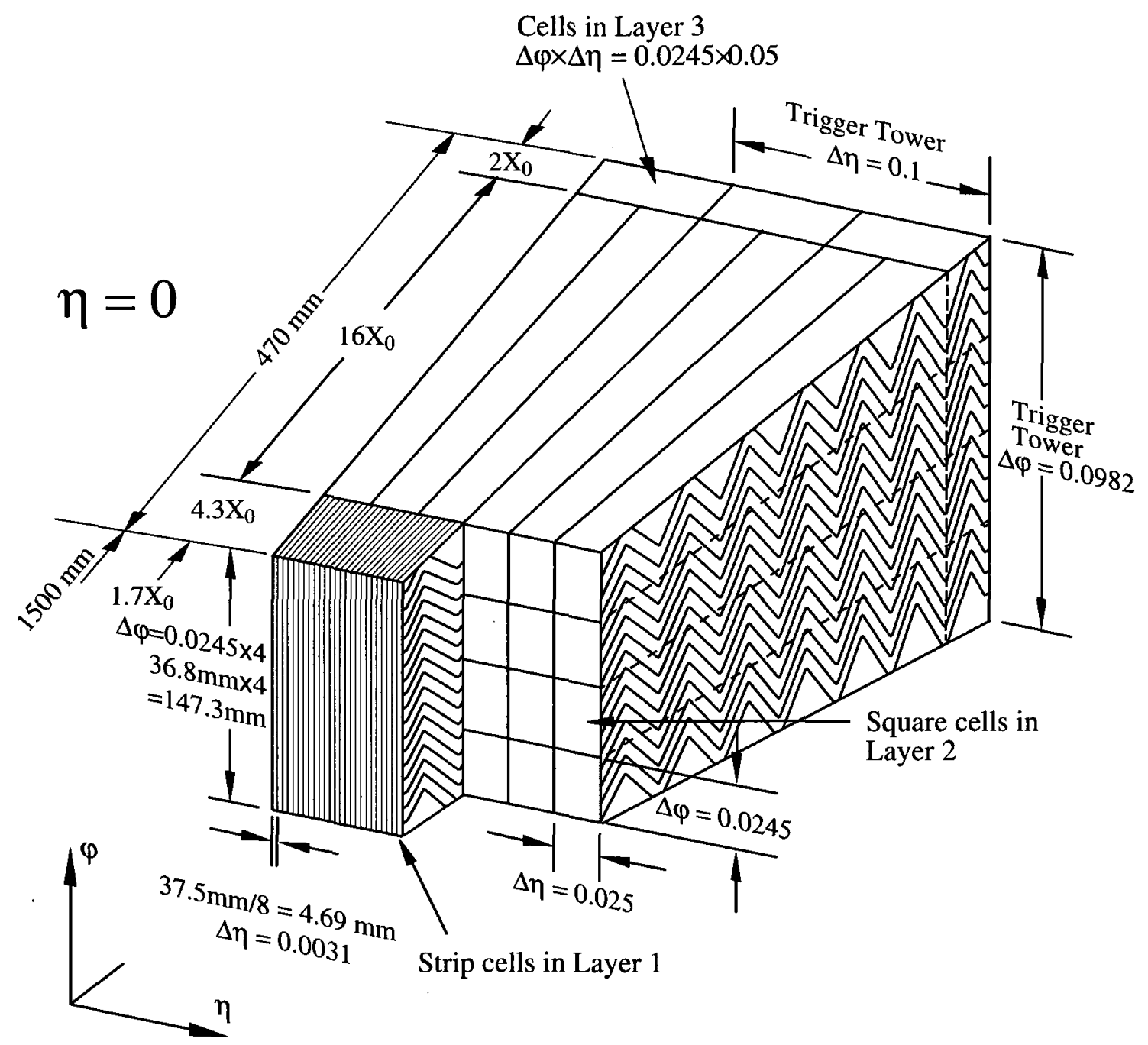

FIgURE 3.7: An electromagnetic barrel calorimeter module, showing the accordion structure. Note also the layout and thicknesses of the three sampling layers and their granularities in $\eta$ and $\phi$. Figure from [13]. 


\begin{tabular}{l||c|r} 
Component & Number of layers & $|\eta|$ coverage \\
\hline \hline Presampler & 1 & $|\eta|<1.52$ \\
\hline Calorimeter & 3 & $|\eta|<1.35$ \\
& 2 & $1.35<|\eta|<1.475$ \\
\hline \hline & Granularity $(\Delta \eta \times \Delta \phi)$ & $|\eta|$ coverage \\
\hline \hline Presampler & $0.025 \times 0.1$ & $|\eta|<1.52$ \\
\hline Calorimeter layer 1 & $0.025 / 8 \times 0.1$ & $|\eta|<1.40$ \\
& $0.025 \times 0.025$ & $1.40<|\eta|<1.475$ \\
\hline Calorimeter layer 2 & $0.025 \times 0.025$ & $|\eta|<1.40$ \\
& $0.075 \times 0.025$ & $1.40<|\eta|<1.475$ \\
\hline Calorimeter layer 3 & $0.050 \times 0.025$ & $|\eta|<1.35$
\end{tabular}

TABLE 3.1: Granularity and $\eta$ coverage of the electromagnetic barrel calorimeter readout.

measures $22 X_{0}-30 X_{0}\left(24 X_{0}-33 X_{0}\right)$ thick from $0<|\eta|<0.8(0.8<|\eta|<1.3)$. Details of the material distribution may be seen in Figure 3.8, while the $\eta$ coverage and granularity are outlined in Table 3.1 . wheel is divided into two coaxial wheels, with the boundary between wheels located at $|\eta|=2.5$. These wheels are further subdivided into eight modules. The inner wheels each contain 256 absorbers and have an active thickness of $26 X_{0}-36 X_{0}$, while each outer wheel contains 768 absorbers and has an active thickness of $24 X_{0}-38 X_{0}$. The $\eta$ coverage and granularity of the endcap calorimeter are outlined in Table 3.2. The endcap and the barrel are separated by a gap in the region $1.447<|\eta|<1.55[30]$. In order to minimize the energy lost in this region, scintillator tiles have been installed 


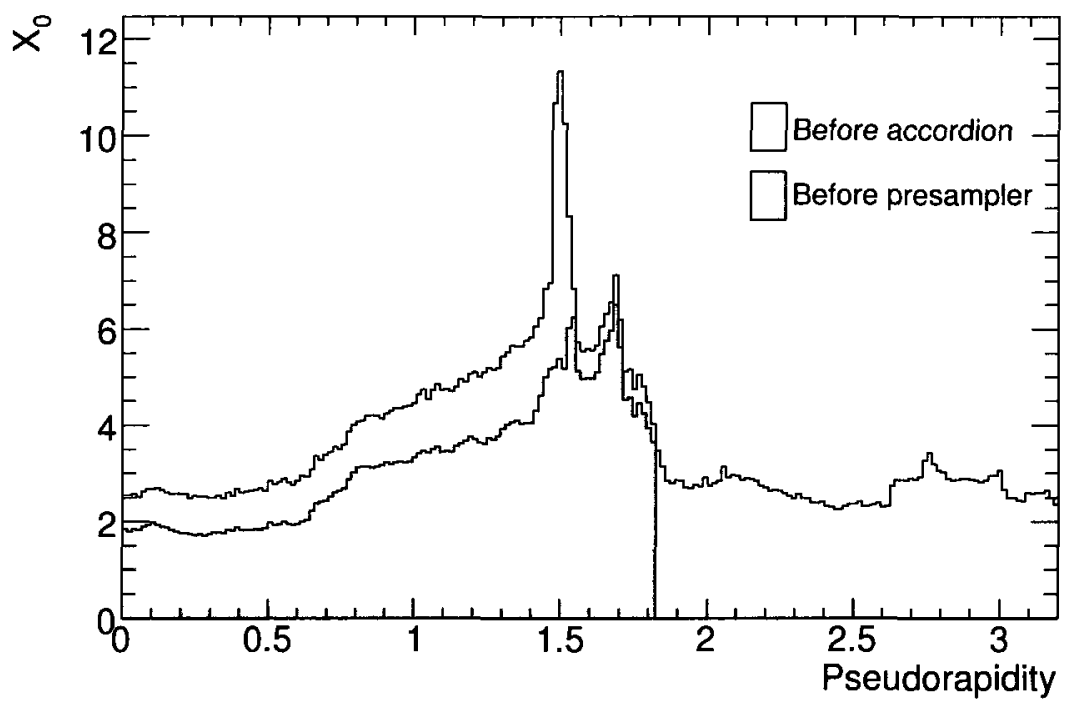

(a) Amount of material in front of the presampler and the accordion.

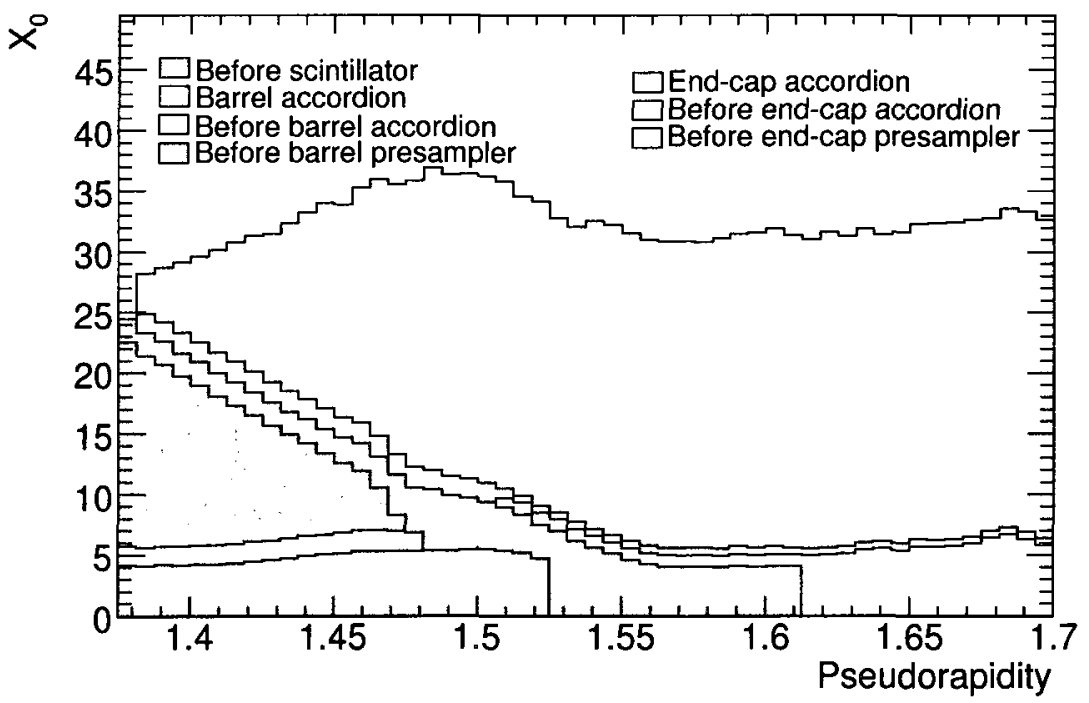

(b) Amount of material in the barrel-endcap crack.

FIGURE 3.8: Cumulative amounts of material, in radiation lengths, in the electromagnetic barrel and endcap calorimeters as a function of $|\eta|$. Figure from [13]. 


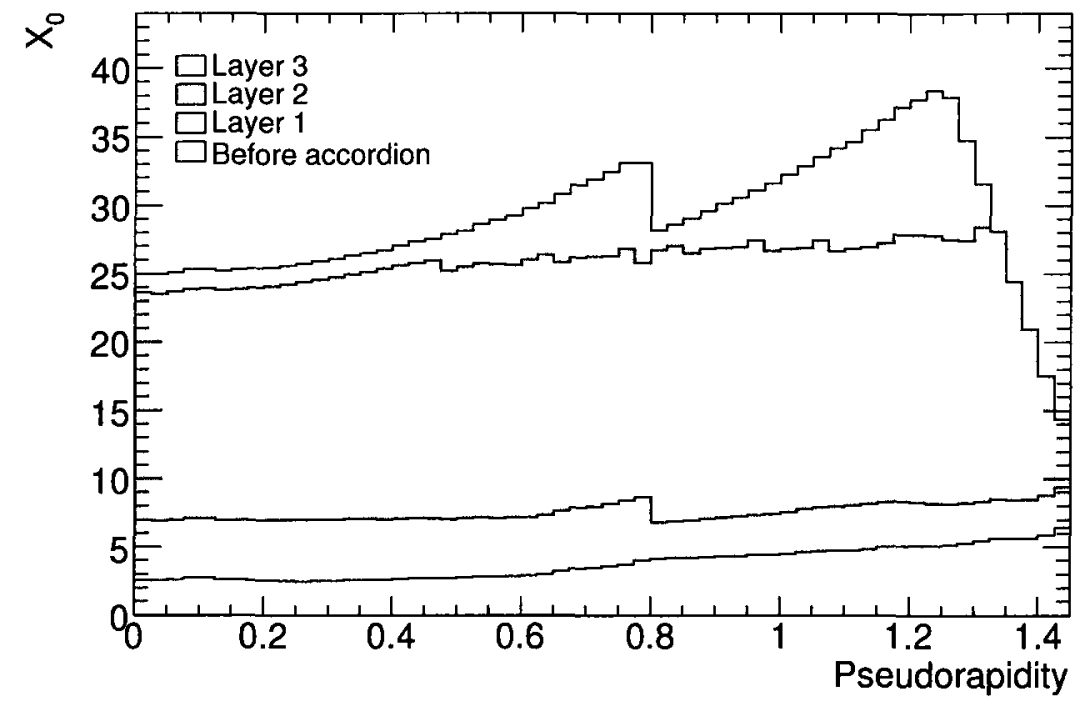

(c) Thickness of each layer of the barrel.

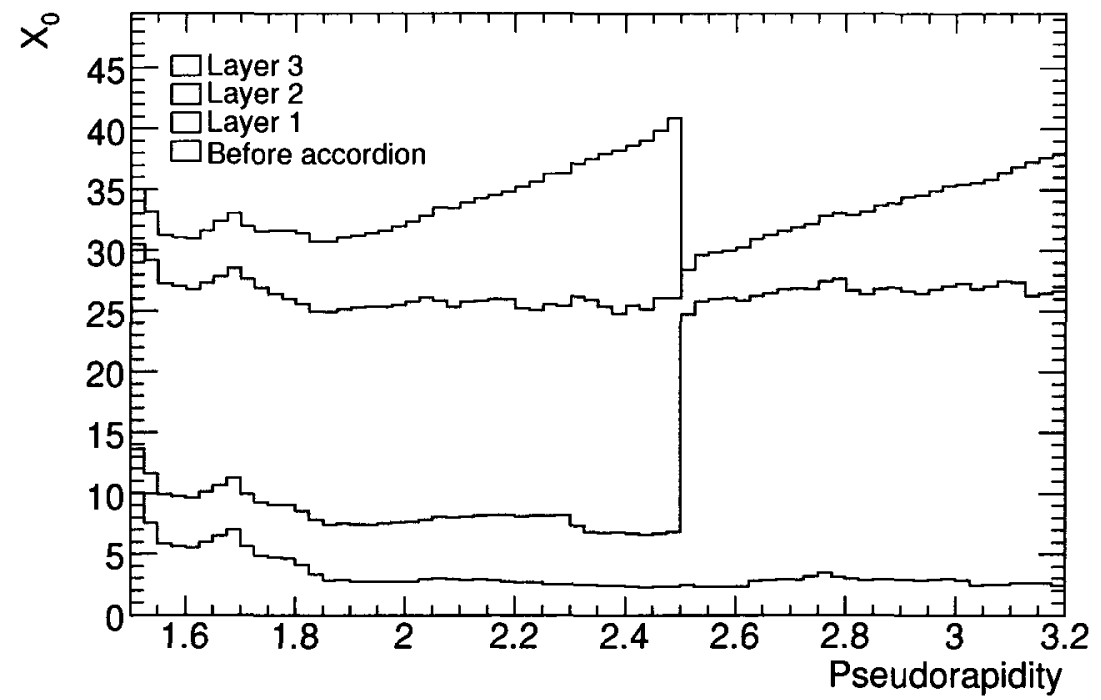

(d) Thickness of each layer of the endcap. 


\begin{tabular}{l||c|r} 
Component & Number of layers & $|\eta|$ coverage \\
\hline \hline Presampler & 1 & $1.5<|\eta|<1.8$ \\
\hline Calorimeter & 2 & $1.375<|\eta|<1.425$ \\
& 3 & $1.5<|\eta|<2.5$ \\
& 2 & $2.5<|\eta|<3.2$ \\
\hline \hline & Granularity $(\Delta \eta \times \Delta \phi)$ & $|\eta|$ coverage \\
\hline \hline Presampler & $0.025 \times 0.1$ & $1.5<|\eta|<1.8$ \\
\hline Calorimeter layer 1 & $0.050 \times 0.1$ & $1.375<|\eta|<1.425$ \\
& $0.025 \times 0.1$ & $1.425<|\eta|<1.5$ \\
& $0.025 / 8 \times 0.1$ & $1.5<|\eta|<1.8$ \\
& $0.025 / 6 \times 0.1$ & $1.8<|\eta|<2.0$ \\
& $0.025 / 4 \times 0.1$ & $2.0<|\eta|<2.4$ \\
& $0.025 \times 0.1$ & $2.4<|\eta|<2.5$ \\
& $0.1 \times 0.1$ & $2.5<|\eta|<3.2$ \\
\hline Calorimeter layer 2 & $0.050 \times 0.025$ & $1.375<|\eta|<1.425$ \\
& $0.025 \times 0.025$ & $1.425<|\eta|<2.5$ \\
& $0.1 \times 0.1$ & $2.5<|\eta|<3.2$ \\
\hline Calorimeter layer 3 & $0.050 \times 0.025$ & $1.5<|\eta|<2.5$
\end{tabular}

TABLE 3.2: Granularity and $\eta$ coverage of the electromagnetic endcap calorimeter readout.

in the gap.

Additional coverage in the region $|\eta|<1.8$ to compensate for upstream energy losses is provided by a presampler consisting of $1.1 \mathrm{~cm}(0.5 \mathrm{~cm})$ of liquid argon in the barrel (endcap). In the barrel, the presampler consists of 32 azimuthal sectors per half-barrel, covering $\Delta \eta \times \Delta \phi=1.52 \times 0.2$ [13]. Each sector contains eight nonidentical modules, consisting of printed circuit board electrodes at $+2000 \mathrm{~V}$, separated by $2 \mathrm{~mm}$ liquid argon gaps. The readout cells, which consist of groups of electrodes, 
have a granularity of $\Delta \eta \times \Delta \phi=0.025 \times 0.1$ [33]. Each endcap presampler contains 32 identical sectors, each consisting of three electrodes separated by two $2 \mathrm{~mm}$ layers of liquid argon [13]. The external electrodes are held at $-2000 \mathrm{~V}$ and the signal is read out from the central electrode.

The barrel (endcap) calorimeter has a total of 101760 (62208) readout channels. There are 7808 (1536) readout channels in the barrel (endcap) presampler. In total, the electromagnetic calorimeter contains 173312 readout channels.

\subsection{Energy Reconstruction}

Electromagnetically interacting particles deposit the majority of their energy in the first and second layers of the calorimeter, with smaller amounts being deposited in the presampler and the third layer. Additionally, small fractions of energy are lost in the material in front of and behind the calorimeter. The development of an electromagnetic shower in the accordion is illustrated in Figure 3.9. Instead of measuring the energy deposited in individual cells, energy is often collected and measured in clusters of cells. In the second layer of the barrel, the cluster area is equivalent to $3 \times 7$ cells in $\eta \times \phi$ for electrons and converted photons (i.e. photons that have produced an $e^{-} e^{+}$pair) or $3 \times 5$ cells for unconverted photons [30]. The total cluster energy is 


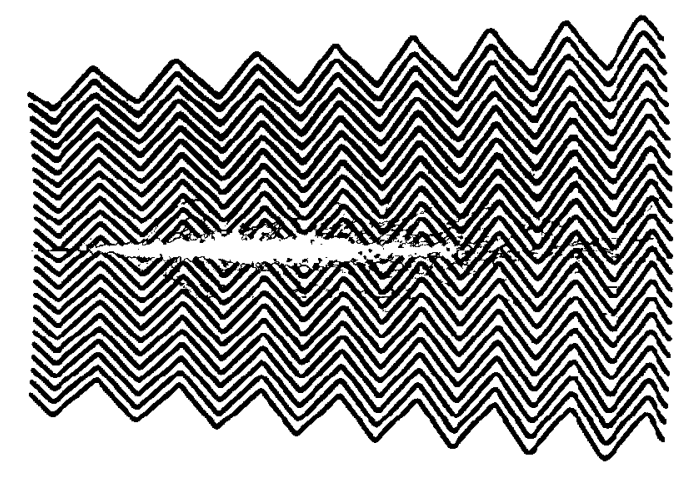

FIgURE 3.9: Schematic of a simulated electromagnetic shower in the accordion. The majority of the energy is deposited in the first and second layers. Figure from [34].

determined by calculating a weighted sum of the energy deposited in the presampler and the three calorimeter layers.

The energy reconstruction method applied to the simulated events used in this work is called the 4-weight method [30]. In this method, the reconstructed energy is calculated as

$$
E_{\text {reco }}=A\left(B+W_{p s} E_{p s}+E_{1}+E_{2}+W_{3} E_{3}\right)
$$

where $E_{p s}$ and $E_{1,2,3}$ are the energies in the presampler and the three calorimeter layers, respectively, $A$ is an overall scale factor and $B$ is an offset term that corrects for upstream energy losses by electrons that do not reach the presampler. $W_{p s}$ and $W_{3}$ are longitudinal weights; the former is applied to the energy deposited by electrons in the presampler to correct for energy lost upstream, while the latter weights the 
energy lost by electrons in the third layer to compensate for energy lost via leakage at the back of the calorimeter.

This parametrisation is not sufficient in the barrel-endcap crack $(1.447<|\eta|<$ 1.55), so the energy in this region is reconstructed using

$$
E_{\text {reco }}=A\left(B+E_{b}+E_{e}+W_{\text {scint }} E_{\text {scint }}\right),
$$

where $E_{b}, E_{e}$ and $E_{\text {scint }}$ are the energies deposited in the barrel and endcap calorimeters and in the scintillators in the barrel-endcap crack, respectively, and $W_{\text {scint }}$ is the longitudinal weight assigned to the energy deposited in the scintillators.

The parameters $A, B, W_{p s}, W_{3}$ and $W_{\text {scint }}$ are calculated with Monte Carlo single particle samples having energies between $10 \mathrm{GeV}$ and $200 \mathrm{GeV}$ using a $\chi^{2}$ minimization of $\left(E_{\text {true }}-E_{\text {reco }}\right)^{2} / \sigma^{2}\left(E_{\text {true }}\right)$, where $E_{\text {true }}$ is the expected energy and $\sigma\left(E_{\text {true }}\right)$ is a parametrisation of the expected energy resolution. This is carried out for bins of $\Delta|\eta|=0.025$, which corresponds to the granularity of the second calorimeter layer, in which the majority of the shower energy is deposited. The weights are functions of $|\eta|$ and do not depend on the energy. Fits are performed separately for electrons and photons and for various cluster sizes. Typical values for these weights are shown in Figure 3.10.

The energy response, given by $\left(E_{\text {reco }}-E_{\text {true }}\right) / E_{\text {true }}$, is shown in Figure 3.11 for 


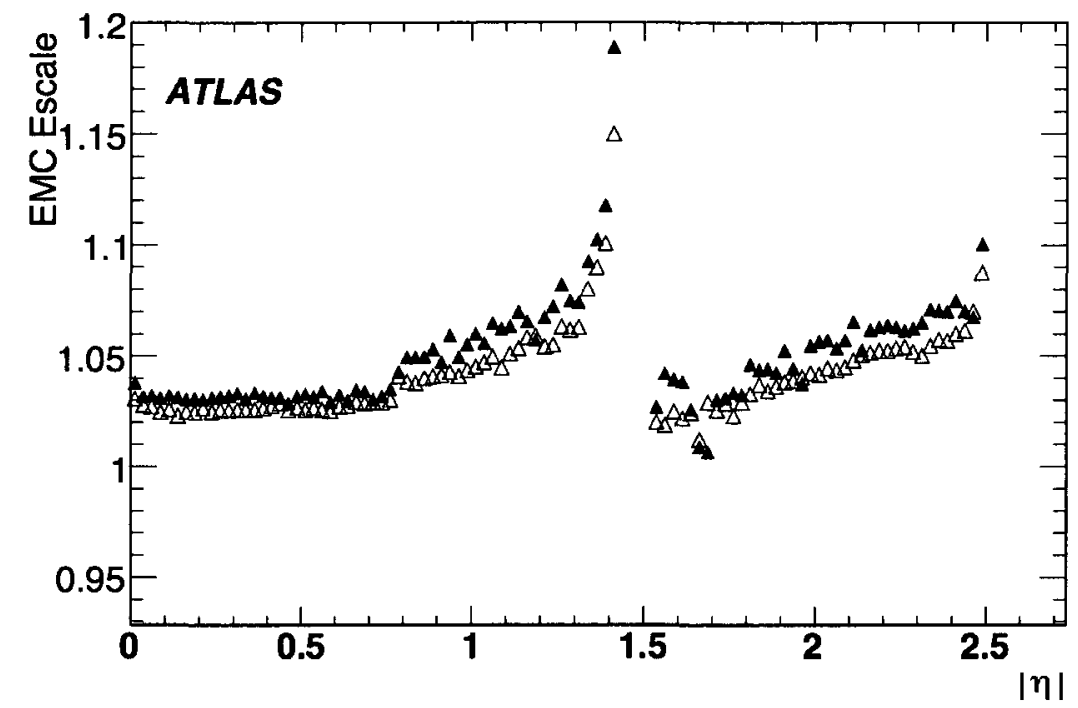

(a) Scale factor $A$.

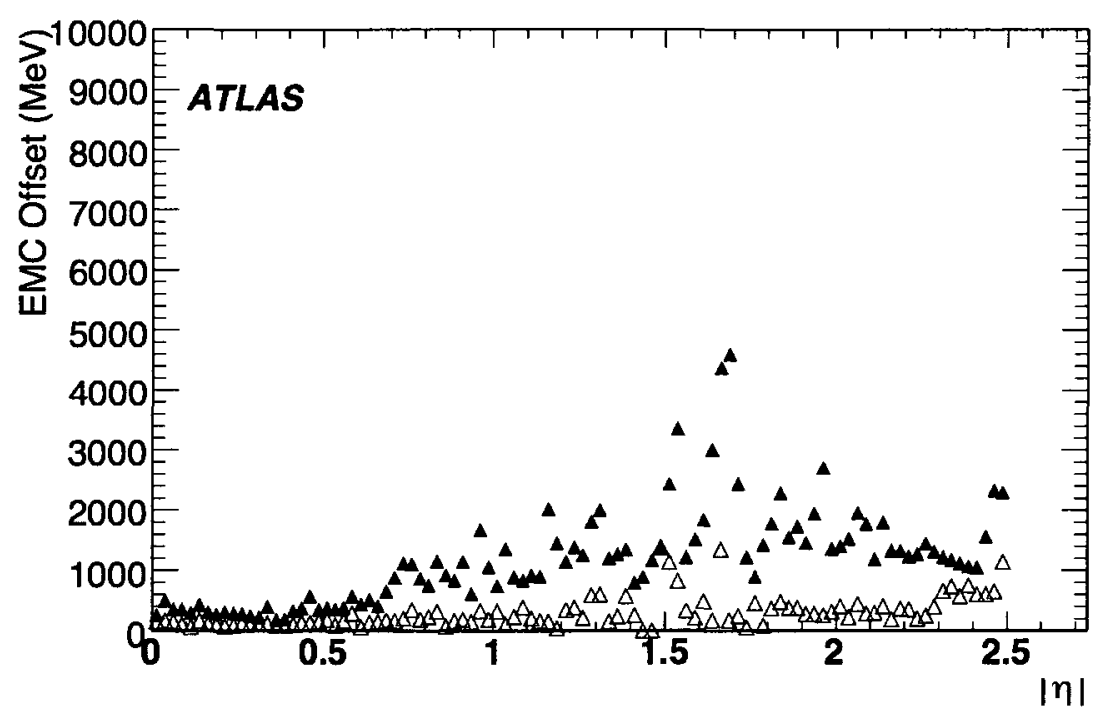

(b) Offset $B$.

FIgURE 3.10: Fitted scale factor $A$ (a), offset $B$ (b) and longitudinal weights $W_{p s}$ (c) and $W_{3}$ (d) for electrons (solid markers) and photons (open markers). The discontinuities at $|\eta| \approx 1.5$ are due to the barrel-endcap crack. Figure from [30]. 


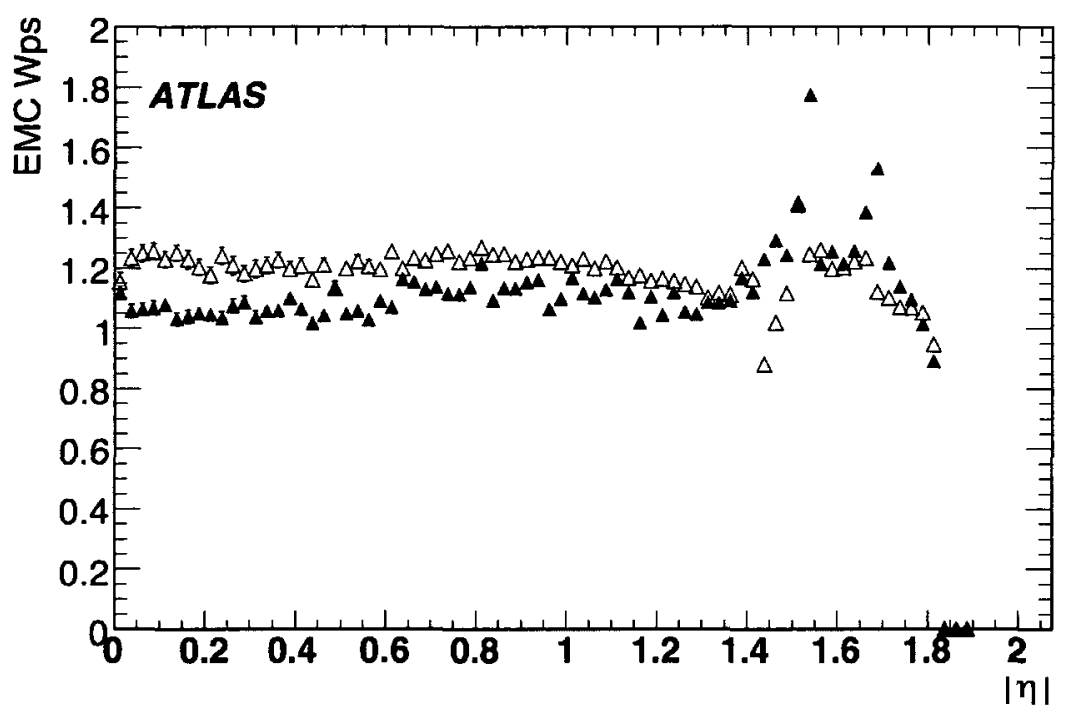

(c) Longitudinal weight $W_{p s}$.

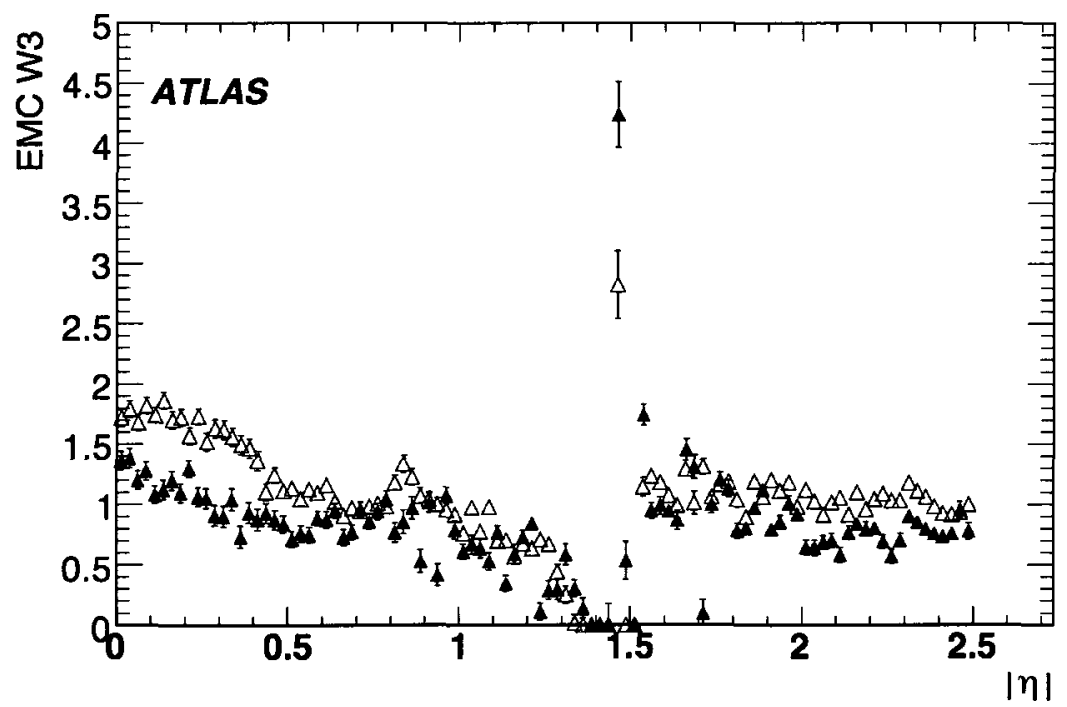

(d) Longitudinal weight $W_{3}$. 


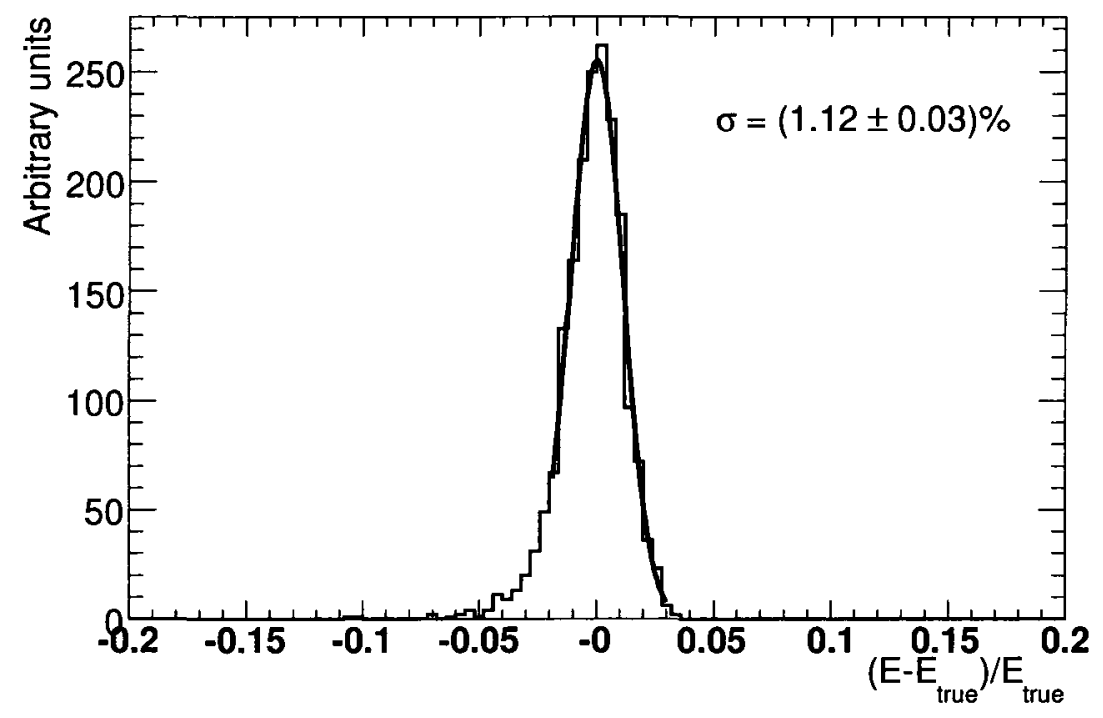

(a) Electrons, $|\eta| \approx 0.325$.

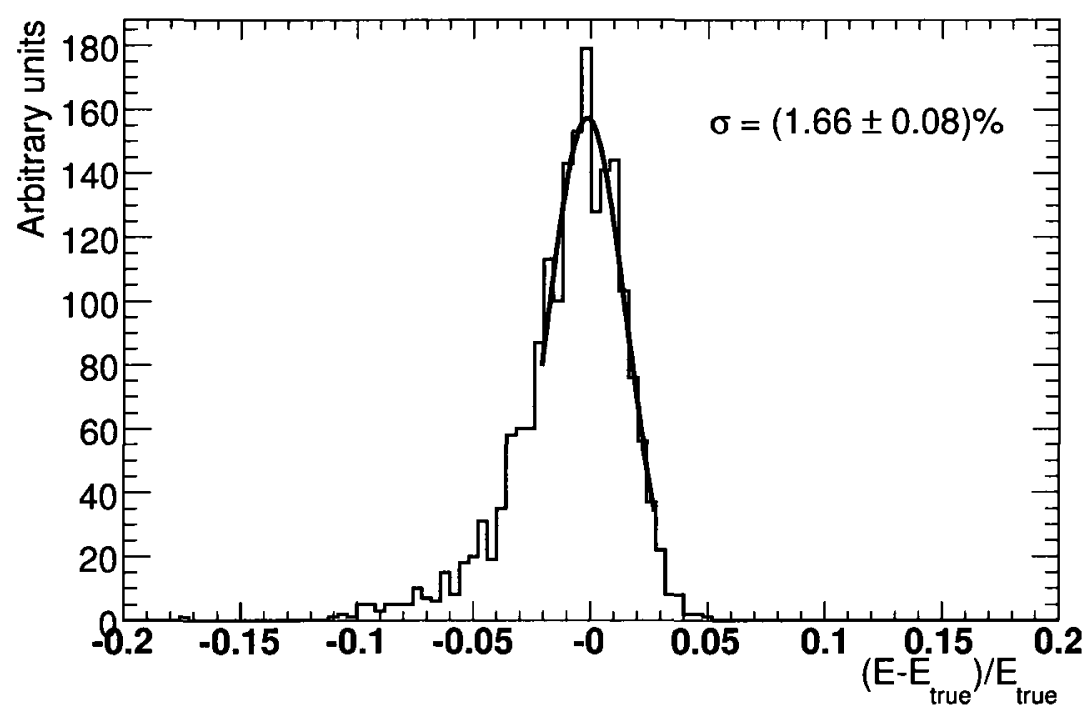

(b) Electrons, $|\eta| \approx 1.075$.

FIGURE 3.11: Energy response of the electromagnetic calorimeter for $100 \mathrm{GeV}$ electrons and photons. Note the degradation of the resolution and the low-energy tail for electrons at higher $|\eta|$, due to the presence of greater amounts of material upstream. The reconstruction at higher $|\eta|$ is slightly better for photons that do not convert. Figure from [13]. 


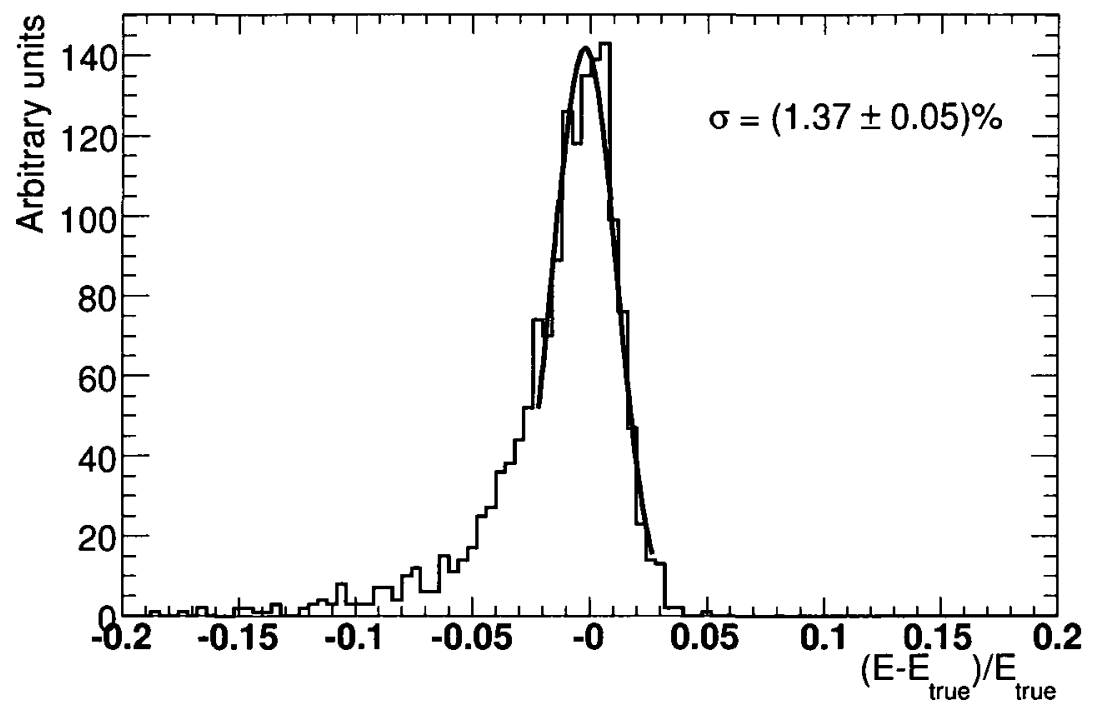

(c) All photons, $|\eta| \approx 1.075$.

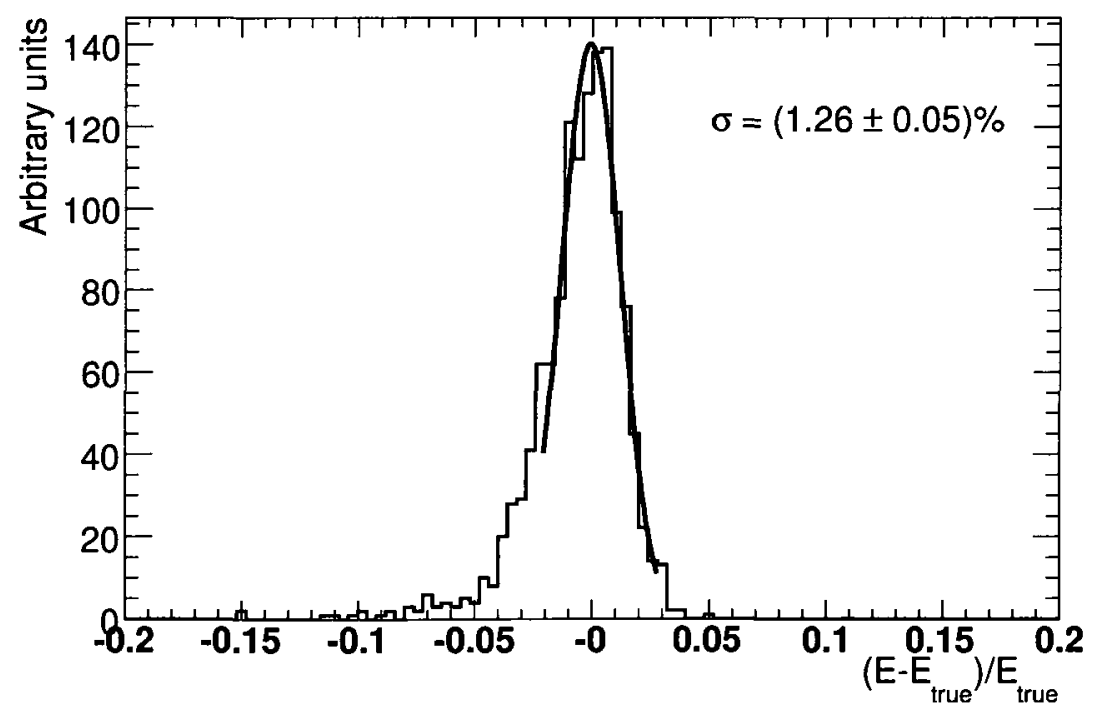

(d) Unconverted photons, $|\eta| \approx 1.075$. 
$100 \mathrm{GeV}$ electrons at two $|\eta|$ positions, and for converted and unconverted photons.

At higher $|\eta|$, the electron energy reconstruction is significantly affected by the larger quantity of material in front of the calorimeter (recall Figure 3.8); this is evident in the non-Gaussian tail and greater width of the distribution. The energy response at higher $|\eta|$ is somewhat better for photons than for electrons, although the Gaussian core and low-energy tail of the distribution are slightly worse for those that undergo conversion, when one compares all photons to unconverted photons.

Test beam measurements have shown that in the barrel, the electron energy resolution, given by Equation 3.10, has a sampling term of $10 \% \cdot \sqrt{\mathrm{GeV}}$ and a constant term $b=0.17 \%$ after subtracting the noise, which is approximately $250 \mathrm{MeV}$ [35]. The resolution in Figure 3.11 is on the order of $1 \%-1.5 \%$, which we would expect from Equation 3.10 for $E=100 \mathrm{GeV}$ with the measured sampling term. The energy response is linear within $\pm 0.1 \%$ in the energy range of $15 \mathrm{GeV} \leq E \leq 180 \mathrm{GeV}$. Similar results were obtained for the endcap [13]. 


\section{Chapter 4}

\section{Impact of Extra Material in Front}

${ }_{\infty}$ of the EM Calorimeter and in the

\section{Inner Detector}

Once ATLAS begins to take data, proper calibration of the electromagnetic calorimeters will be of crucial importance in order to assure optimal performance in reconstructing electrons and photons. The reconstructed energy, given by Equation 3.11, includes corrections (the offset $B$ and the longitudinal weight $W_{p s}$ ) for the energy lost by electrons as they encounter material upstream of the calorimeter. This necessitates the development of a precise model of the material distribution in and around 
Chapter 4. Impact of Extra Material in Front of the EM Calorimeter and in the Inner Detector

the calorimeter, as the presence of extra material (e.g. the inner detector, cables, electronics, support structures, etc.) can alter the development and shape of electromagnetic showers, and can have a detrimental impact on the energy response of the calorimeter. Recall from Figure 3.8 that the amount of material in the calorimeter increases as a function of $|\eta|$. Figure 4.1 illustrates how the energy resolution and the sampling term of the resolution (refer to Equation 3.10) are degraded as $|\eta|$ (i.e. the amount of material) increases. This, in turn, influences the energy calibration. order of $1 \%$ of a radiation length. This is of particular importance for reconstructing electrons in the inner detector, since electrons lose, on average, $20 \%$ to $50 \%$ of their energy while passing through this subdetector, as shown in Figure 4.2. Detailed studies, including the precise weighing of the inner detector components [13] and a test beam analysis of the calorimeter $[35,36]$, have already been performed, but it will still be necessary to fine-tune the calibration with real data and with the detector in its final configuration when the LHC is restarted.

A method for determining the impact of extra material on shower shapes has been introduced by F. Hubaut et al. [16]. With this method, the shapes of simulated electromagnetic showers initiated by high $p_{T}$ electrons from $W \rightarrow e \nu$ decays in a distorted ATLAS geometry are compared, with the intent of determining the amount and location of extra material in front of the calorimeter. Several of the results 
Chapter 4. Impact of Extra Material in Front of the EM Calorimeter and in the Inner Detector

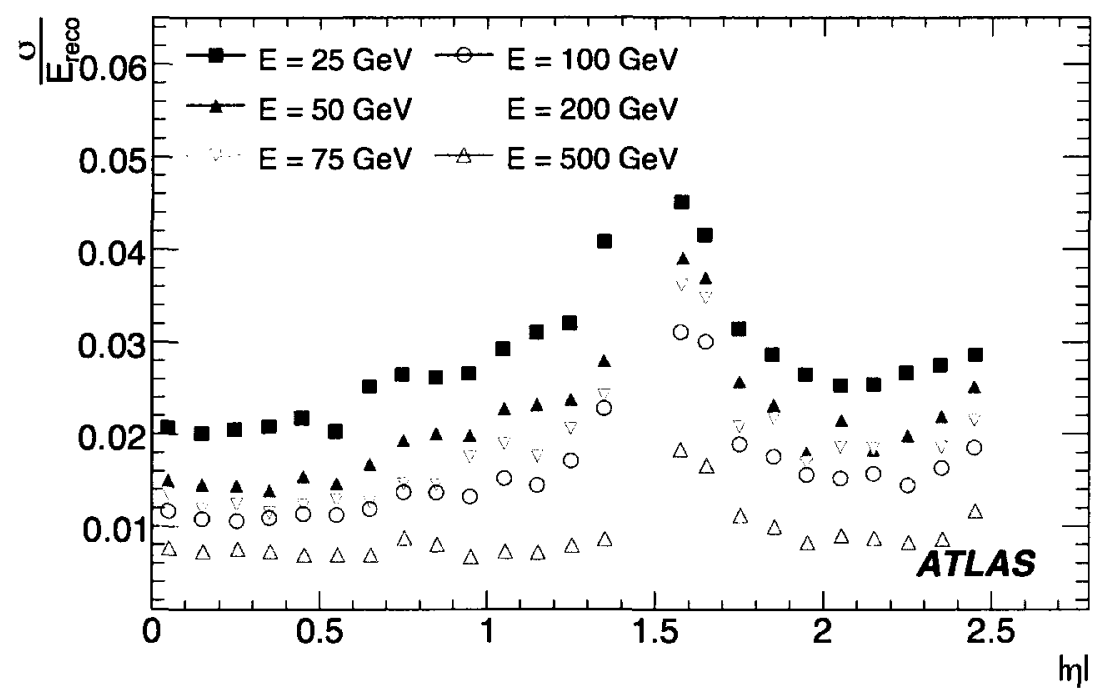

(e) Photon energy resolution.

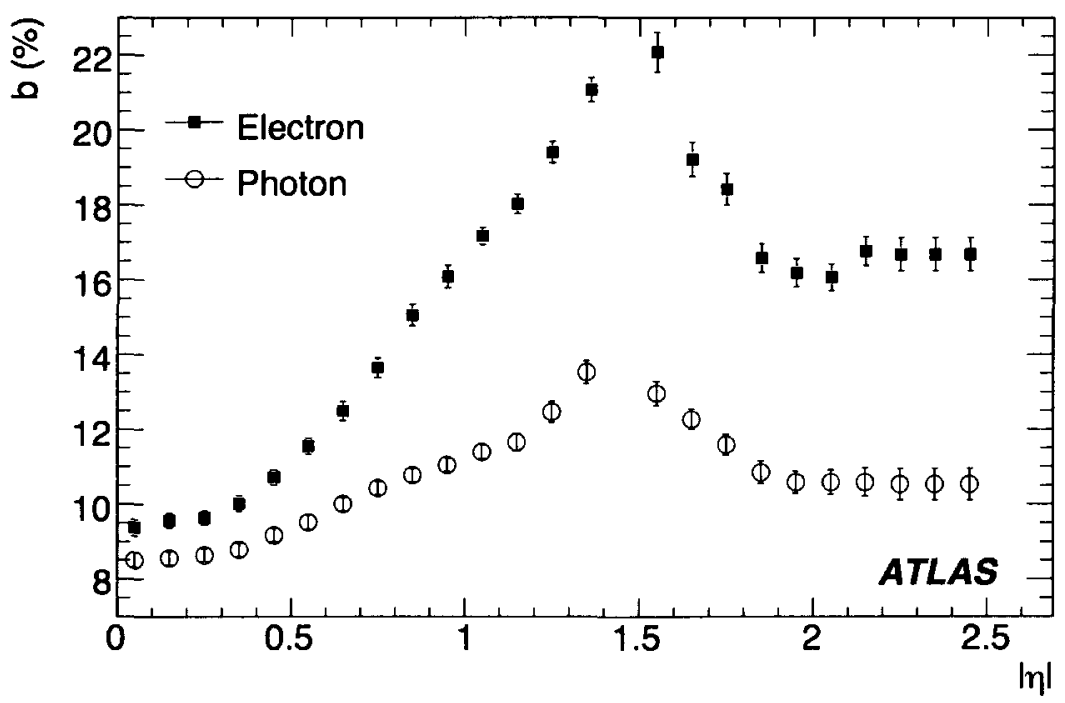

(f) Sampling term.

FIGURE 4.1: Degradation of the energy resolution $\left(\sigma / E_{\text {reco }}\right)$ and the sampling term of the resolution as a function of $|\eta|$. In (a), the points corresponding to $E=200$ $\mathrm{GeV}$ are barely visible due to the colour chosen by the authors of the note in which the plot originally appeared. Figure from [30]. 
Chapter 4. Impact of Extra Material in Front of the EM Calorimeter and in the Inner Detector

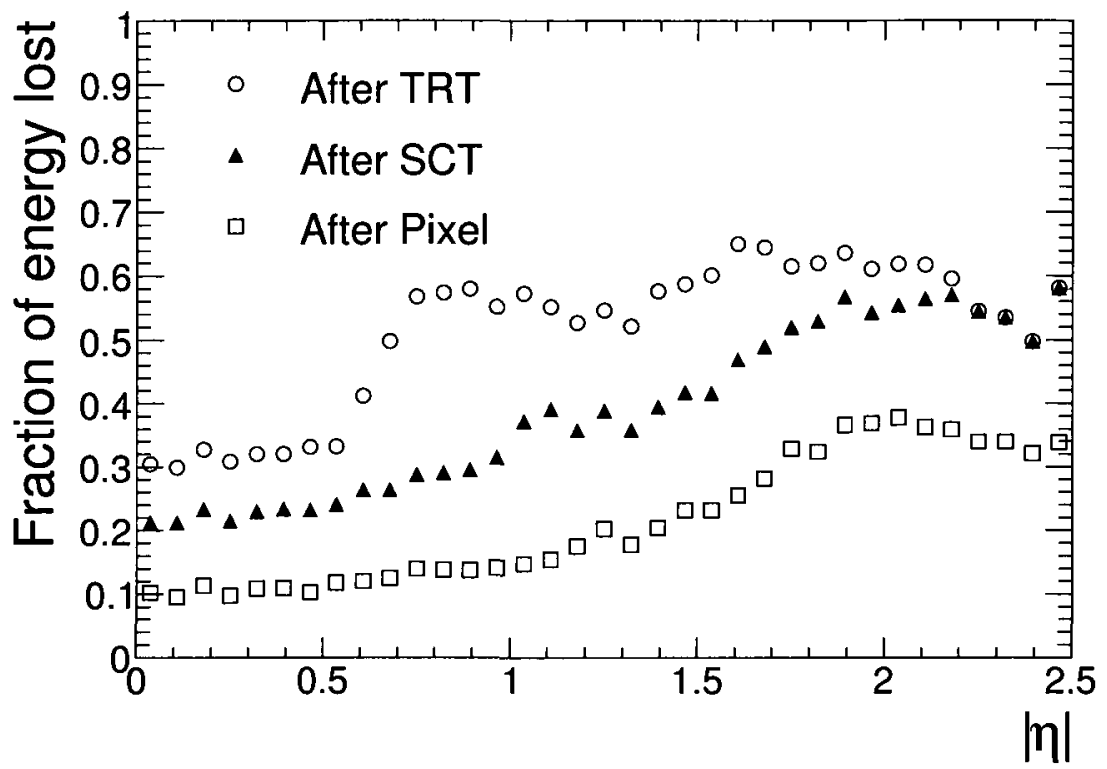

Figure 4.2: Average energy loss of electrons $\left(\left\langle p_{T}\right\rangle=25 \mathrm{GeV}\right)$ in the three inner detector subsystems as a function of $|\eta|$. Figure from [30].

obtained by Hubaut et al. with this method are reproduced below; these results are then used to formulate a more detailed study of the impact of extra material on the longitudinal development of electromagnetic showers in the calorimeter.

An official ATLAS Monte Carlo simulation, featuring a distorted detector geometry, was used to produce the electrons. The properties of electromagnetic showers initiated by these electrons are compared for several regions of the detector in order to determine their effectiveness as discriminating variables to distinguish extra material in the inner detector and in front of the electromagnetic calorimeter. The utility of a new sensitive variable, derived from a combination of shower properties, is also investigated. Since the material composition of the detector should be fully understood 
Chapter 4. Impact of Extra Material in Front of the EM Calorimeter and in the Inner Detector

as early in the data-taking period as possible, the feasibility of repeating this study with initial LHC data is checked. Finally, Monte Carlo truth information related to the occurrence of bremsstrahlung in the inner detector is used to further substantiate the results of this work.

\subsection{Detector Geometry and Simulation of Events}

\subsubsection{Detector Geometry}

For the purposes of this study, a distorted, misaligned geometry, known as CSC-0102-00 [37] is used. The misalignments occur in the inner detector; individual subdetector components are independently rotated or translated within expected build tolerances [30]. The distortion is achieved by dividing the detector into quadrants and simulating extra material in the form of aluminum, carbon and copper in several regions of the detector [37], as outlined below. The simulated detector also contains a reference region reflecting an up-to-date description of the material location and composition.

In the inner detector (ID), no extra material is added in the hemisphere $-\pi<$ $\phi<0$, which serves as the reference region, while the hemisphere $0<\phi<\pi$ serves as the distorted region. In $\eta$, the amount of extra material varies between $0.02 X_{0}$ 
Chapter 4. Impact of Extra Material in Front of the EM Calorimeter and in the Inner Detector

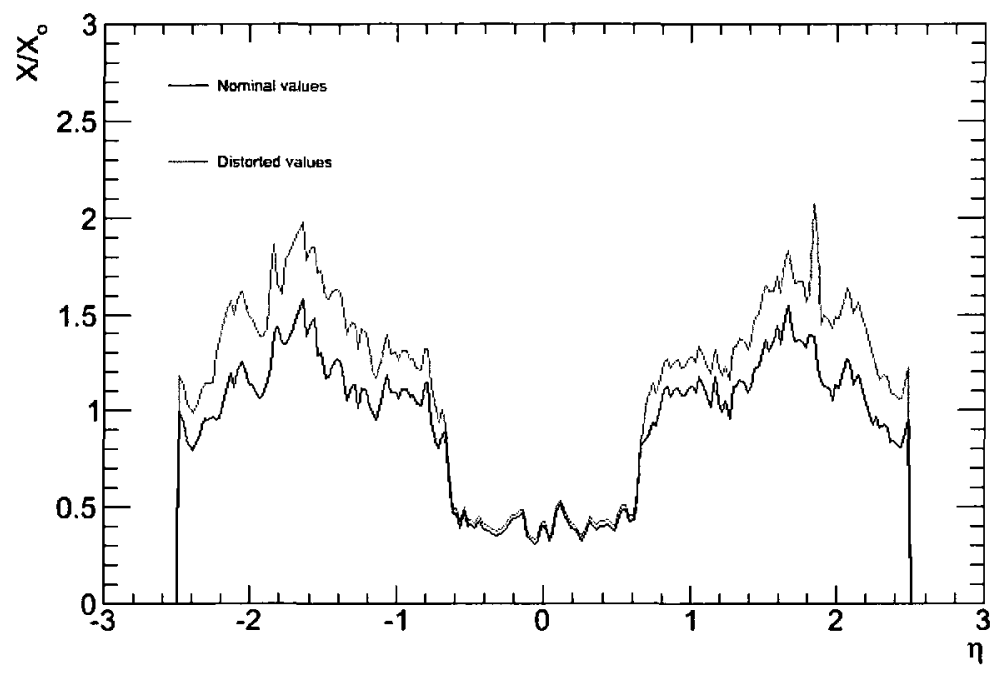

FIGURE 4.3: Inner detector material distribution (in number of radiation lengths) as a function of $\eta$.

and $0.5 X_{0}$, as shown in Figure 4.3. The nominal layout of the inner detector is illustrated in Figure 4.4. Figure 4.5 reproduces this schematic, but with the simulated extra material depicted in red. In the barrel, material is added between the first and second pixel and SCT layers, while in the endcap, material is added around the SCT and the TRT.

In front of the electromagnetic barrel calorimeter, $5 \%$ of a radiation length of extra material has been added between the presampler (PS) and the first sampling layer (S1), as well as $10 \%$ of a radiation length in the cryostat. The material has been added radially, so the quantity must be multiplied by $\cosh \eta$ to simulate the amount of material actually encountered by particles coming from the interaction point. The 
Chapter 4. Impact of Extra Material in Front of the EM Calorimeter and in the
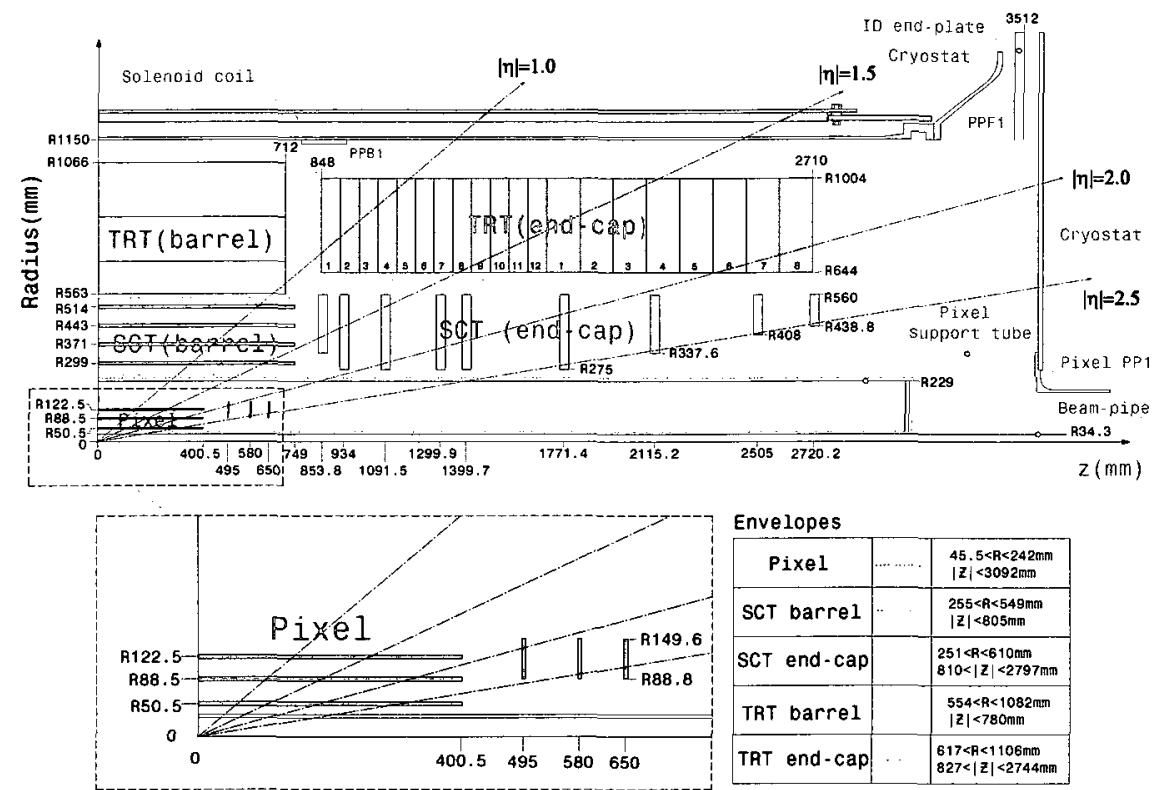

Figure 4.4: Nominal inner detector layout in $R-z$. Figure from [13].

amount and location of the extra calorimeter material is summarized in Table 4.1 and illustrated in Figure 4.6.

By choosing two appropriate quadrants in $\phi$ and comparing so-called "shower variables" (described below) in those quadrants, it is possible to disentangle the effect of extra material in a particular region. The reference and distorted quadrants that are used for comparison purposes are displayed in Table 4.2 and illustrated in Figure 4.7.

\subsubsection{High- $p_{T}$ Electrons}

High $p_{T}$ electrons from $W \rightarrow e \nu$ will be one of the first clean sources of isolated electrons during early running. (Note that charge conjugation is assumed, but for 
Chapter 4. Impact of Extra Material in Front of the EM Calorimeter and in the Inner Detector

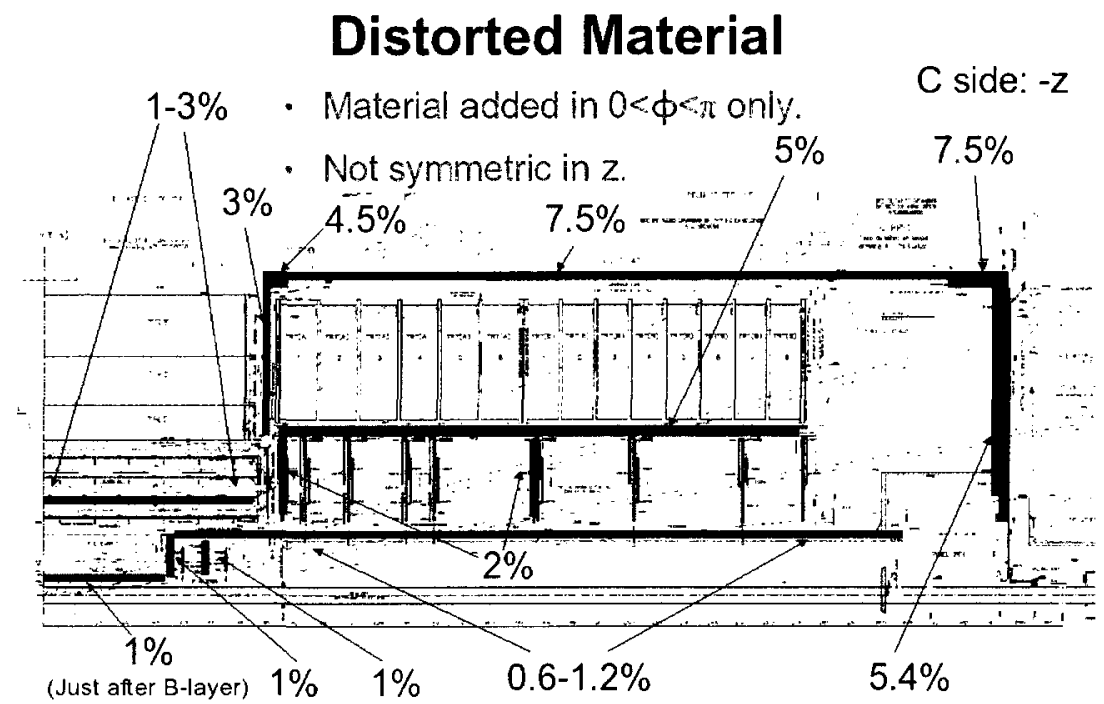

(a) Side $C(z<0)$.

\section{Distorted Material}

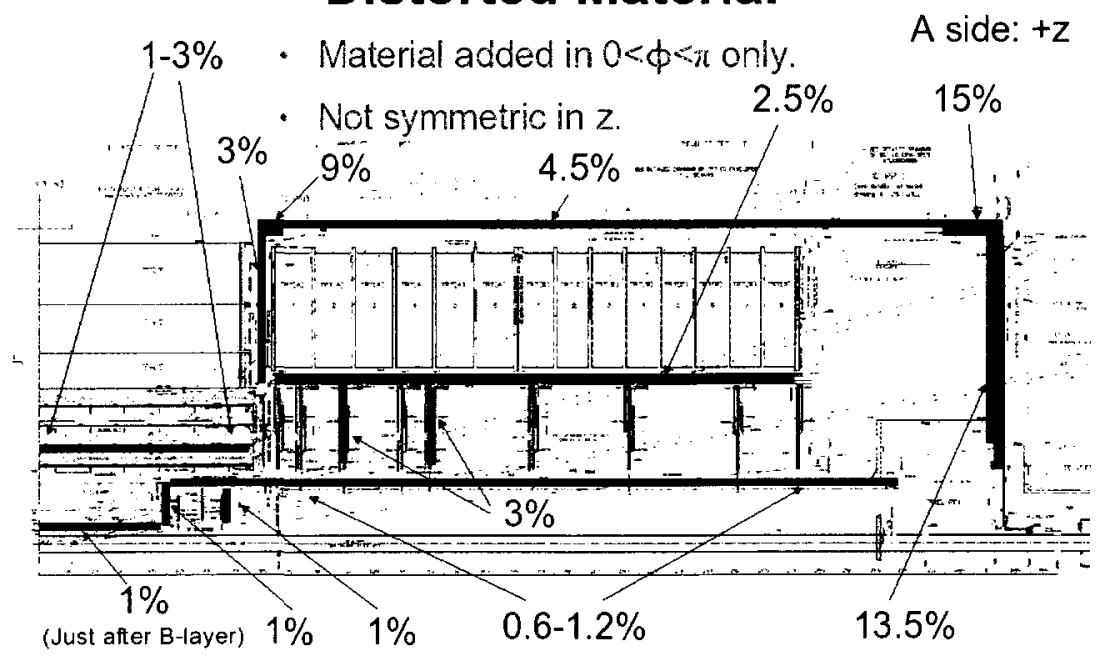

(b) Side A $(z>0)$.

Figure 4.5: Extra inner detector material distribution (red bars) in $R-z$. The amount of material added is expressed as a percentage of a radiation length. Compare with Figure 4.4. Figure from [37]. 
Chapter 4. Impact of Extra Material in Front of the EM Calorimeter and in the Inner Detector

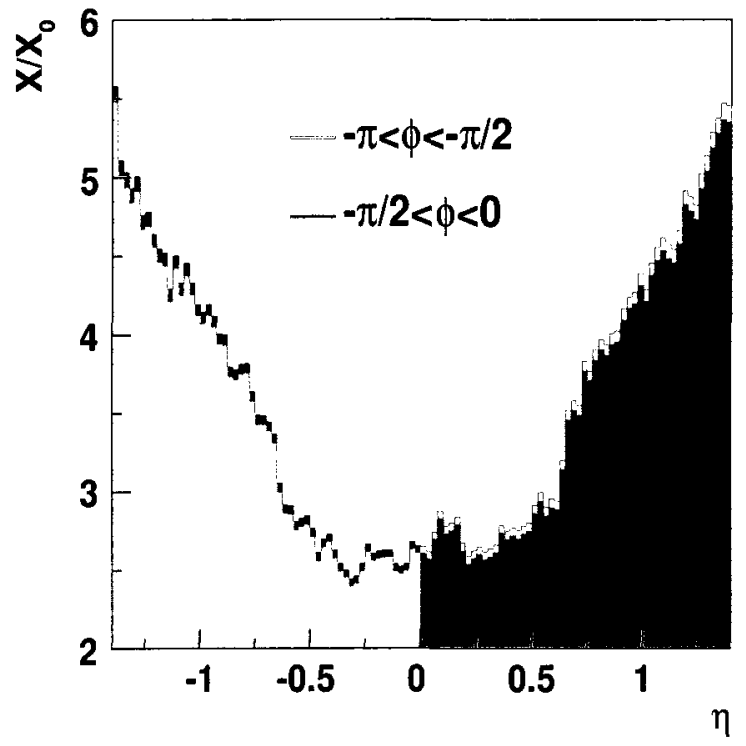

FIGURE 4.6: Electromagnetic barrel calorimeter material distribution as a function of $\eta$. Figure from [16].
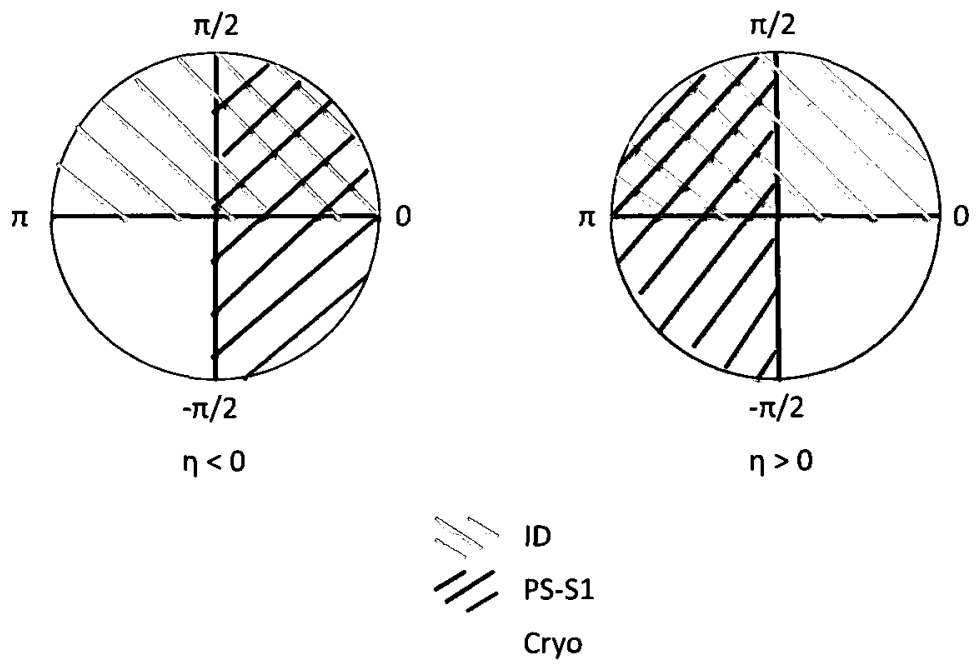

FIguRE 4.7: Schematic of the distribution of extra material in the inner detector and in front of the electromagnetic calorimeter. 
Chapter 4. Impact of Extra Material in Front of the EM Calorimeter and in the Inner Detector

\begin{tabular}{c||c|r} 
& $\frac{\pi}{2}<\phi<\frac{3 \pi}{2}$ & $-\frac{\pi}{2}<\phi<\frac{\pi}{2}$ \\
\hline \hline$\eta<0$ & 0 & $0.05 \cdot \cosh \eta($ PS-S1) \\
\hline$\eta>0$ & $0.05 \cdot \cosh \eta(\mathrm{PS}-\mathrm{S} 1)+0.10 \cdot \cosh \eta(\mathrm{Cryo})$ & $0.10 \cdot \cosh \eta($ Cryo $)$
\end{tabular}

TABLE 4.1: Amount of material, in radiation lengths $\left(X_{0}\right)$, added in front of the electromagnetic barrel calorimeter in the CSC-01-02-00 geometry (as traversed by a particle coming from the interaction point).

\begin{tabular}{l||c|c} 
Extra material & Reference region & Distorted region \\
\hline \hline ID & $-\pi<\phi<-\frac{\pi}{2}(\eta<0)$ & $\frac{\pi}{2}<\phi<\pi(\eta<0)$ \\
\hline PS-S1 & $-\pi<\phi<-\frac{\pi}{2}(\eta<0)$ & $-\frac{\pi}{2}<\phi<0(\eta<0)$ \\
\hline Cryo & $-\pi<\phi<-\frac{\pi}{2}(\eta<0)$ & $-\frac{\pi}{2}<\phi<0(\eta>0)$
\end{tabular}

TABLE 4.2: Summary of distorted and reference regions in the CSC-01-02-00 geometry. Each distorted region contains extra material in or around one subdetector only, while the reference regions contain no extra material. By comparing shower properties in the distorted and reference regions for one particular location, the impact of extra material in that location can be disentangled.

brevity, positrons will also be called electrons for the purposes of this work.) This study uses approximately 2.5 million simulated $W \rightarrow e \nu$ events, corresponding to roughly $475 \mathrm{pb}^{-1}$ of raw data from proton-proton collisions at $10 \mathrm{TeV}$, assuming a cross section of $14357 \mathrm{pb}$. This figure was derived by assuming that roughly $60 \%$ of events pass acceptance cuts, and that $60 \%$ of these events pass trigger cuts, implying that approximately 6.8 million events would be needed at the generation level in order to identify 2.5 million events.

Electrons are selected based on clusters having $p_{T}>15 \mathrm{GeV}$ and $|\eta|<2.47$, 
Chapter 4. Impact of Extra Material in Front of the EM Calorimeter and in the Inner Detector

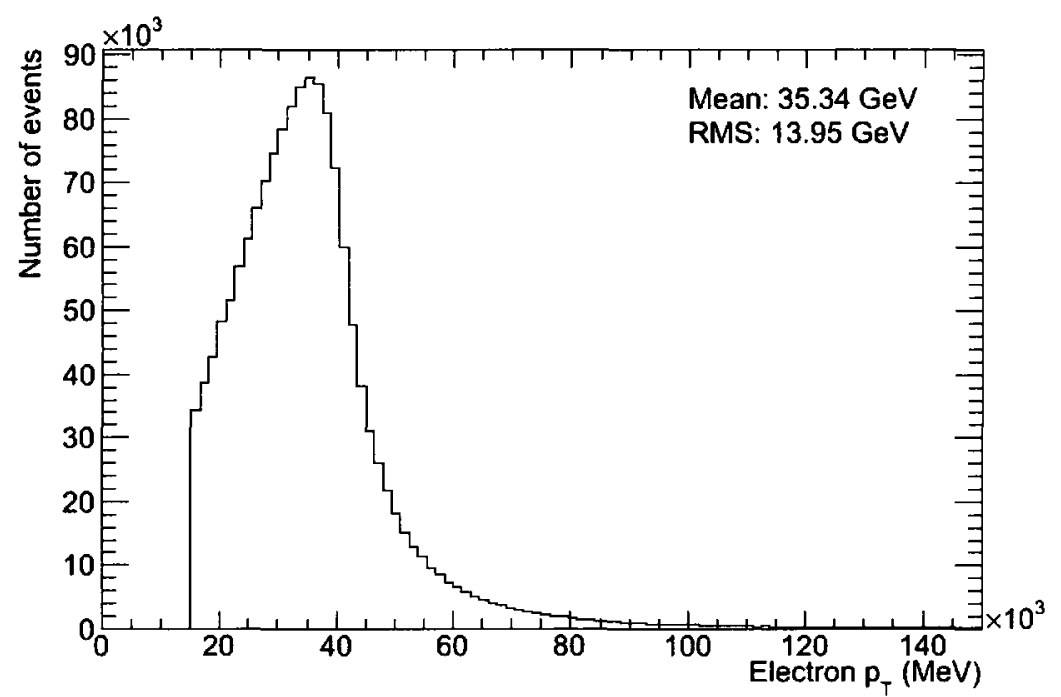

Figure 4.8: $p_{T}$ distribution of the simulated electron sample.

excluding the barrel-endcap crack region and the adjacent material $(1.35<|\eta|<$ 1.52). Distributions of the electrons' $p_{T}, E$ and locations in $\eta$ and $\phi$ are found in Figure 4.8, Figure 4.9, Figure 4.10 and Figure 4.11. The electrons are identified using the so-called "medium" cuts [30], which consider the shower width and the energy deposited in the first and second layers of the calorimeter, as well as tracking variables such as the number of hits in the pixels and in the SCT. After all cuts are taken into account, approximately 1.5 million electrons remain. All results shown in this chapter are based on this statistic.

While Hubaut et al. focused on both the transverse and the longitudinal aspects of electromagnetic showers, this work investigates primarily the longitudinal 
Chapter 4. Impact of Extra Material in Front of the EM Calorimeter and in the Inner Detector

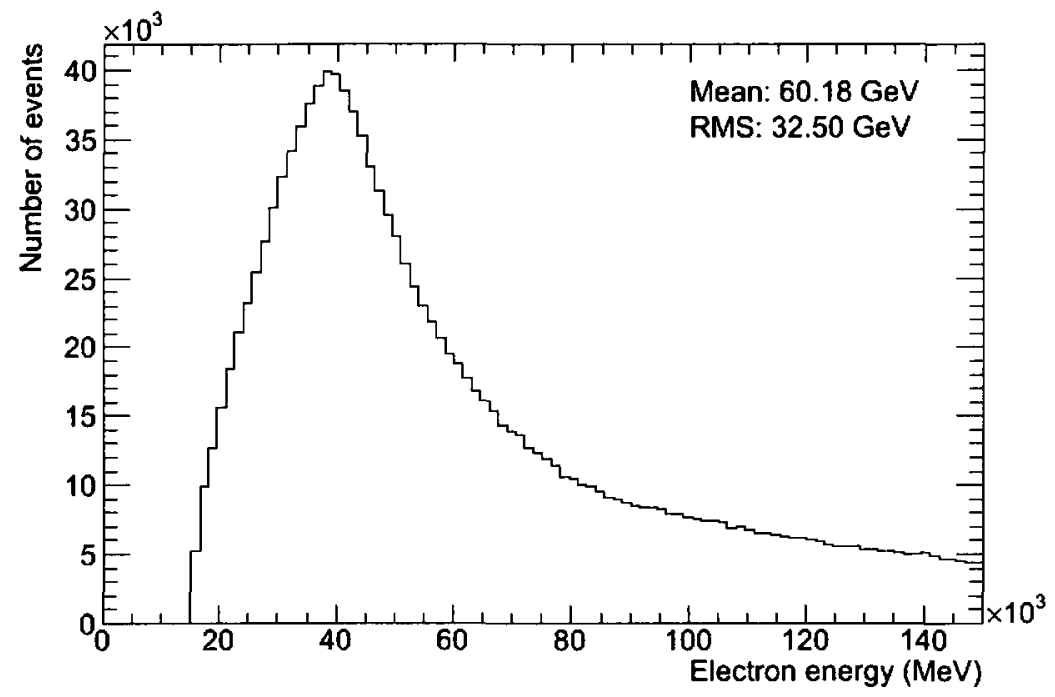

FIGURE 4.9: $E$ distribution of the simulated electron sample.

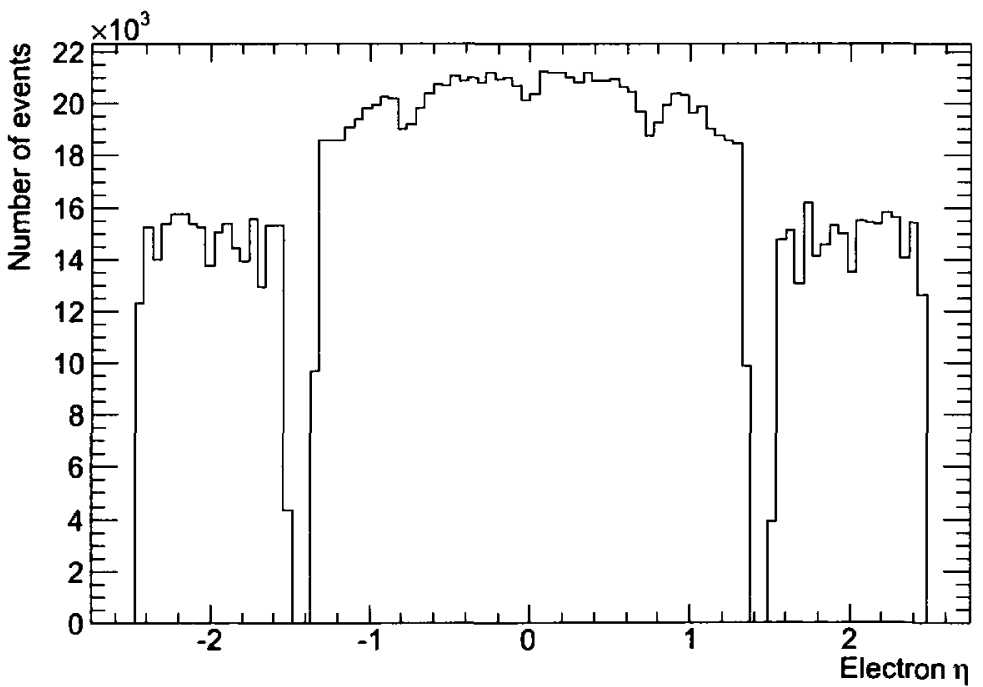

FiguRE 4.10: $\eta$ distribution of the simulated electron sample. 


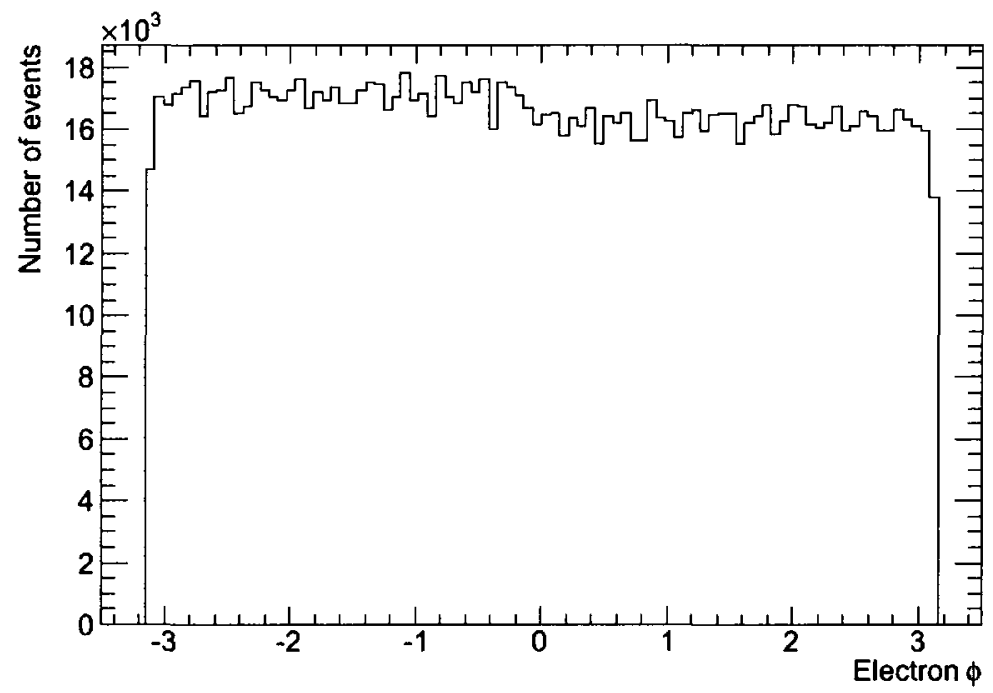

FIGURE 4.11: $\phi$ distribution of the simulated electron sample.

properties. Hence, the shower variables most commonly used are the fraction of energy deposited in each layer (PS, S1, S2, S3) of the calorimeter. Other variables, including the reconstructed and Monte Carlo truth energies and the electrons' energy/momentum ratio are also investigated in this study.

\subsection{Energy Reconstruction}

As discussed in Section 3.3, excellent energy resolution is of great importance, especially in searches for rare processes, such as those involving the Higgs or SUSY, as well as for precision measurements of Standard Model properties. We have already 
Chapter 4. Impact of Extra Material in Front of the EM Calorimeter and in the Inner Detector

is degraded at high values of $|\eta|$ due to the electrons and photons passing through larger amounts of material in the nominal geometry. To confirm some of the results of the original study by Hubaut et al. [16], the effect of extra ID material on the energy scale and energy resolution was investigated.

The relative energy difference between the reconstructed and true energies, given by $\left(E-E_{\text {true }}\right) / E_{\text {true }}$ was plotted for the hemispheres with $(0<\phi<\pi)$ and without $(-\pi<\phi<0)$ extra ID material, in intervals of $\Delta \eta=0.2$. Examples of typical distributions for low and high $\eta$ slices are shown in Figure 4.12. At high $\eta$, it is apparent that the presence of extra material in the distorted region results in the reconstructed energy being lower than the true energy; the extra material also has a detrimental impact on the resolution and contributes to the low-energy tail.

The energy scale, given by the mean of the fit to the Gaussian portion of the distribution, as shown in Figure 4.12, and the fractional energy resolution, given by the RMS of the fit, were then determined for each region in $\eta$. Note that the distorted and reference regions also contain extra material in the cryostat and between the PS and $\mathrm{S} 1$, but for a given slice in $\eta$, both regions contain the same amount of material in these two locations, and so only the impact of the extra ID material is visible. The differences in energy scale and fractional energy resolution between the distorted and reference regions are shown in Figure 4.13 and Figure 4.14, along with the results of the original study by Hubaut et al. Neglecting the area around the crack region 


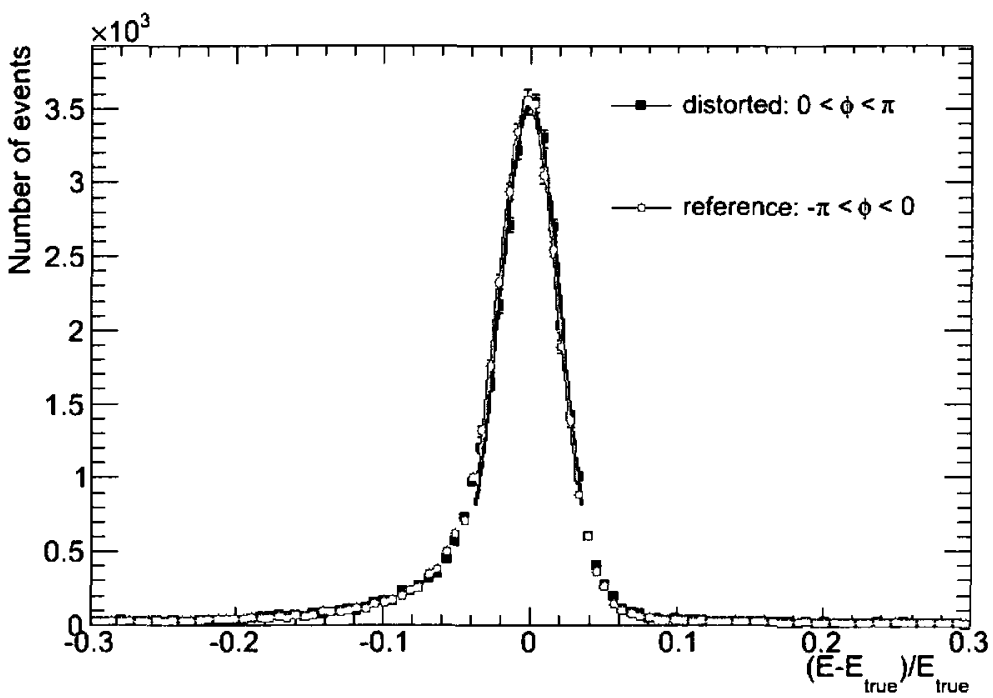

(a) $\eta \approx 0.0$

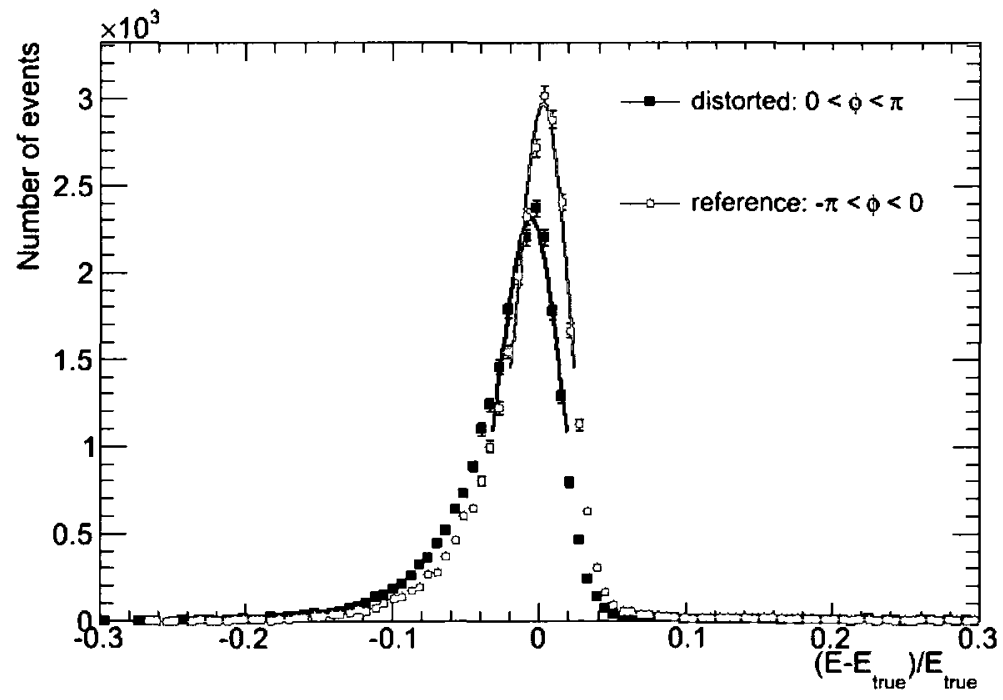

(b) $\eta \approx 2.0$.

FIGURE 4.12: Relative difference between reconstructed and true electron energy at $\eta \approx 0.0$ (left) and $\eta \approx 2.0$ (right) for the regions with and without extra material in the inner detector. The curves are the results of Gaussian fits to the distributions in the peak regions. 
Chapter 4. Impact of Extra Material in Front of the EM Calorimeter and in the

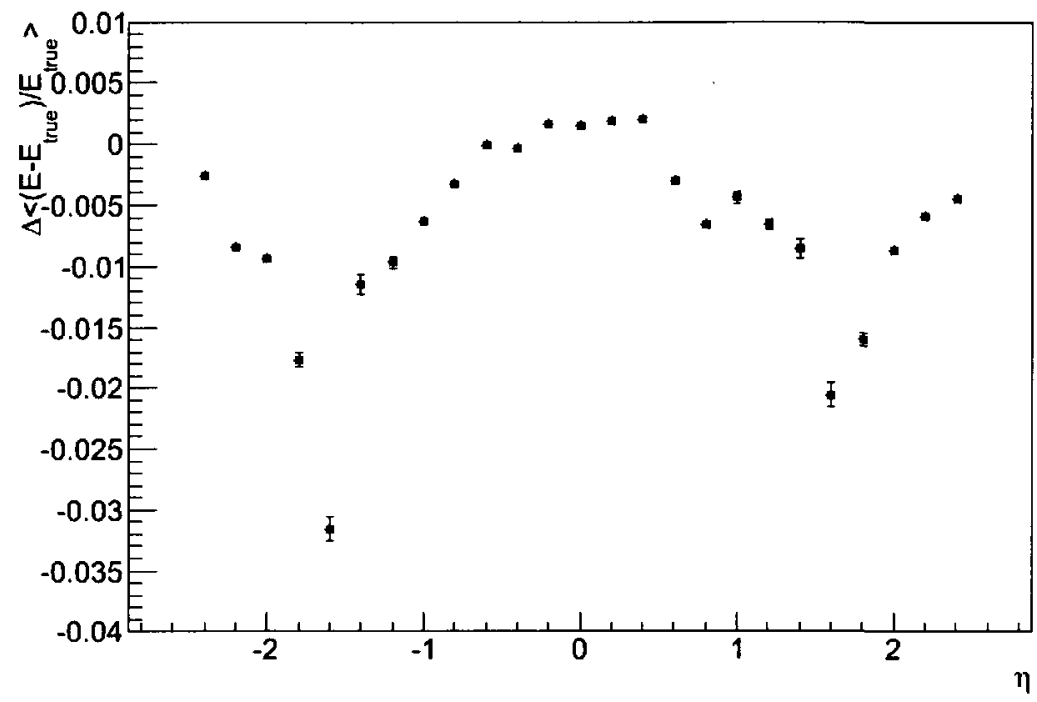

(a) Difference in energy scale.

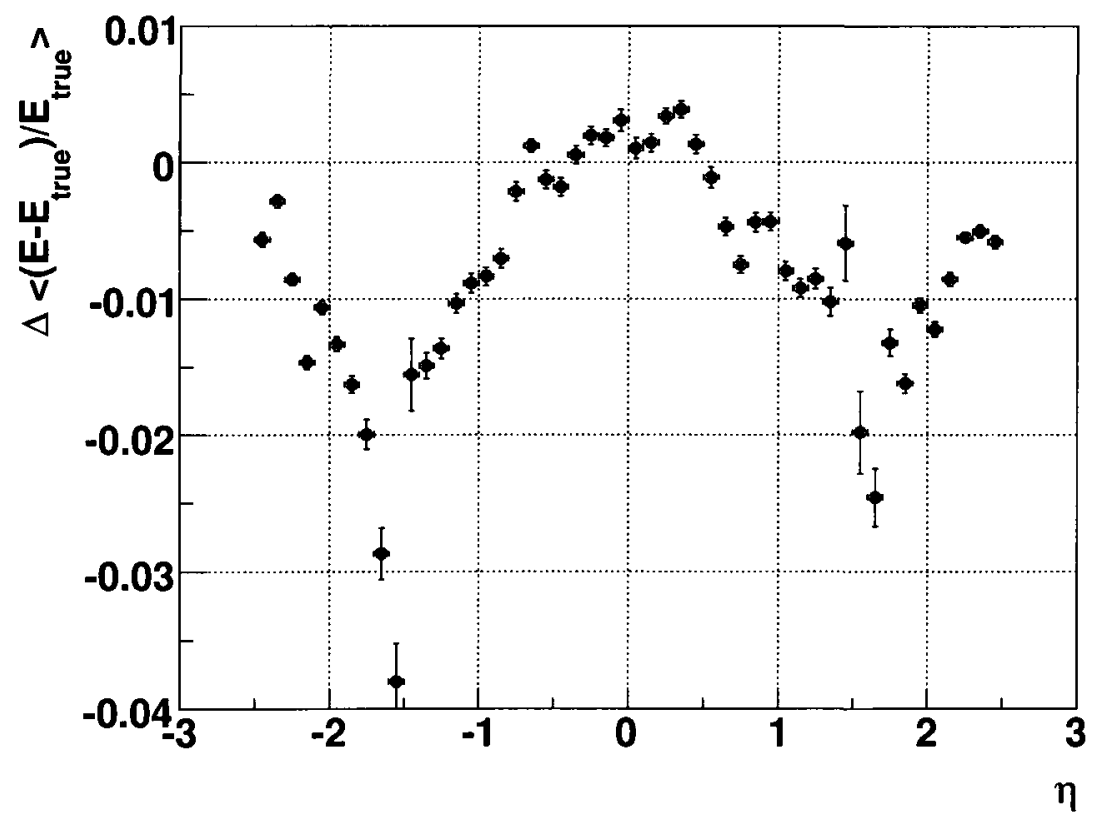

(b) Difference in energy scale from [16].

FIgURE 4.13: Impact of extra ID material on energy scale (a). For comparison purposes, the results of Hubaut et al. [16] are also shown (b). 
Chapter 4. Impact of Extra Material in Front of the EM Calorimeter and in the

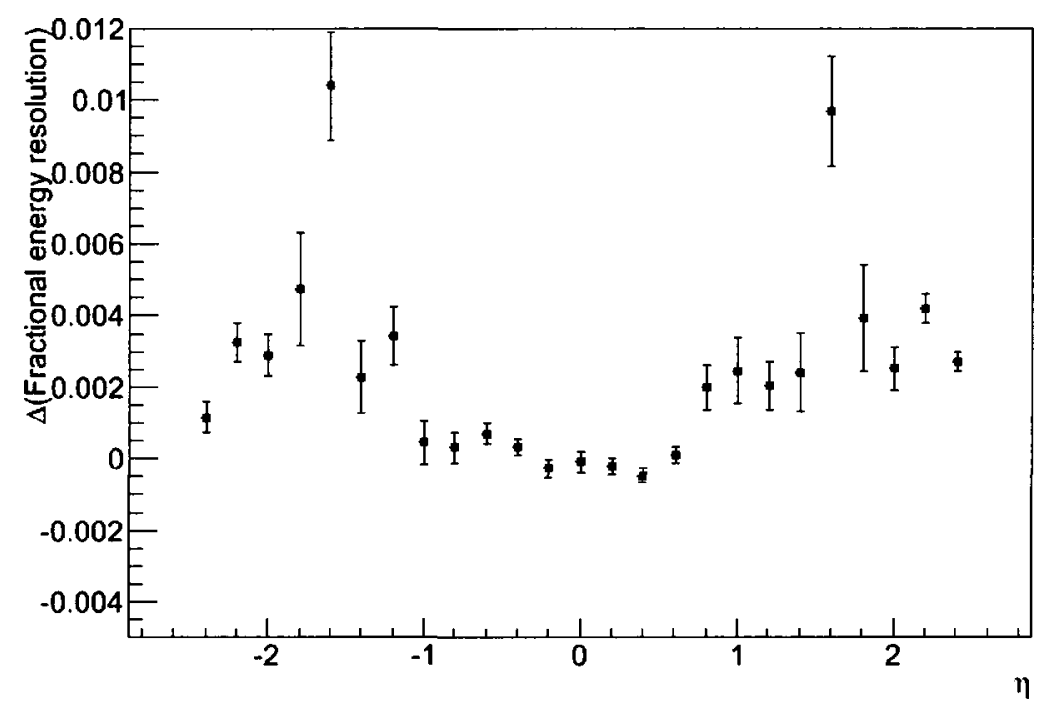

(a) Difference in fractional energy resolution.

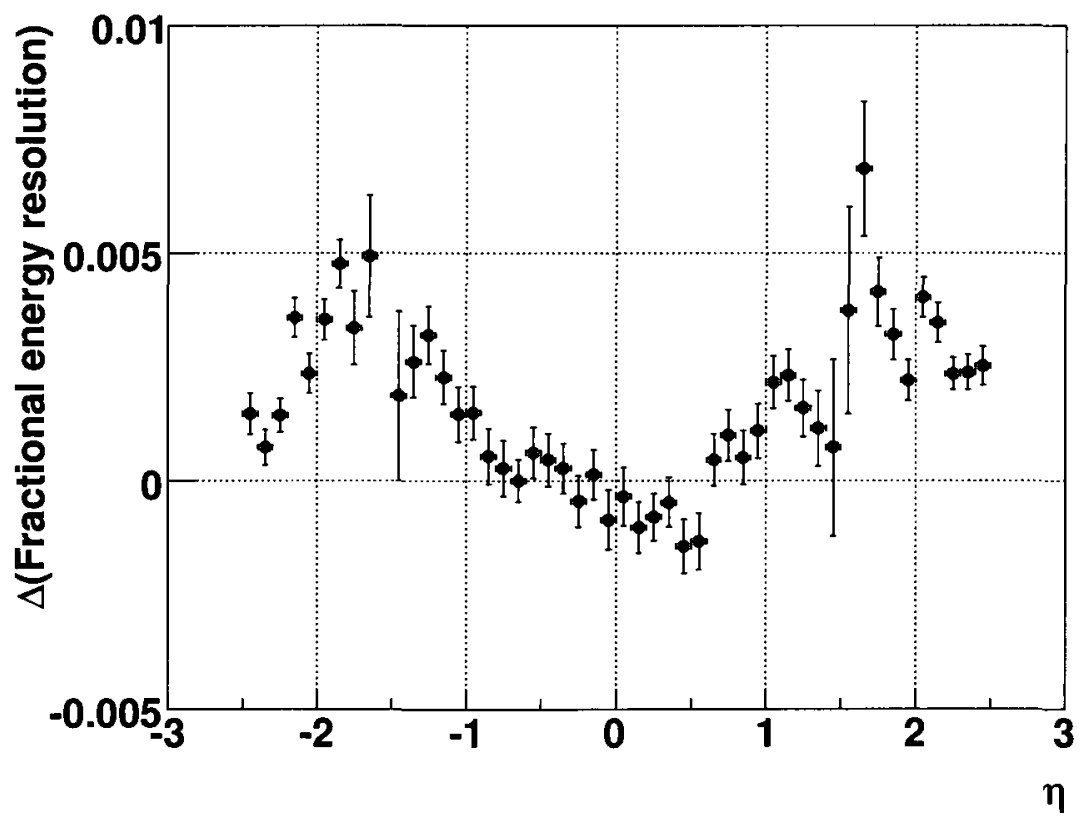

(b) Difference in fractional energy resolution from [16].

FIgURE 4.14: Impact of extra ID material on fractional energy resolution (a). For comparison purposes, the results of Hubaut et al. [16] are also shown (b). 
Chapter 4. Impact of Extra Material in Front of the EM Calorimeter and in the Inner Detector

$(1.35<|\eta|<1.52)$, the energy scale is underestimated by up to $2 \%$, while the energy resolution is degraded by up to $0.4 \%$. These results agree with those obtained by Hubaut et al.

\subsection{Longitudinal Energy Deposition}

At $\eta=-1.15$, the amount of material in S1 decreases, resulting in a smaller fraction of energy being deposited in that layer. Events occurring in the barrel-endcap crack region (corresponding to $-1.35<\eta<-1.52$ ) have been removed. Finally, the PS 
Chapter 4. Impact of Extra Material in Front of the EM Calorimeter and in the Inner Detector

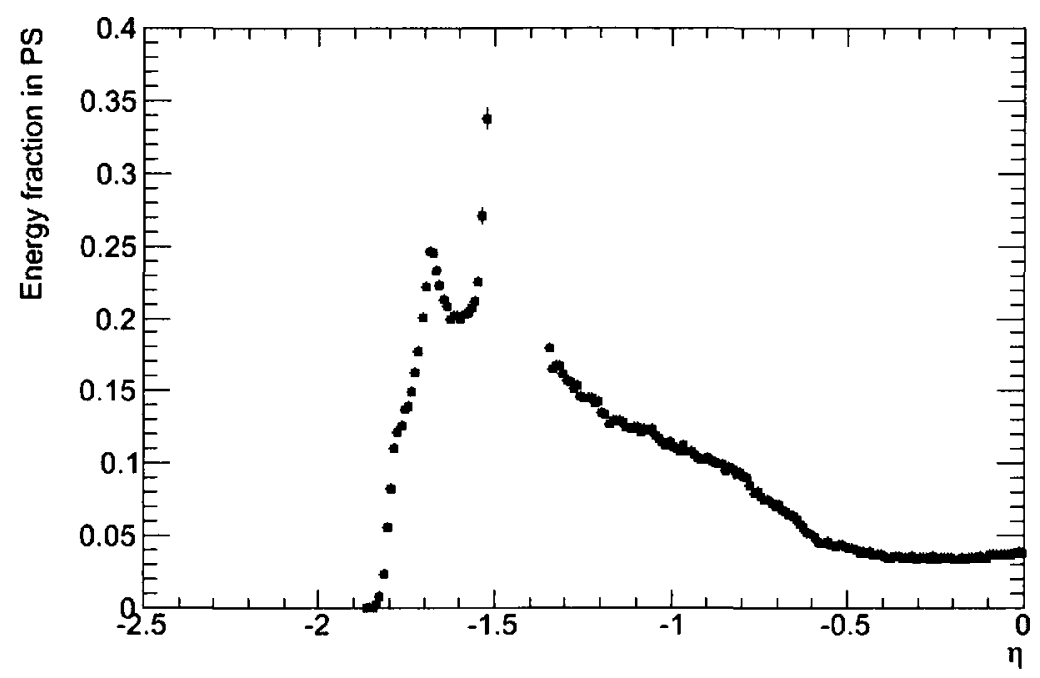

(a) PS.

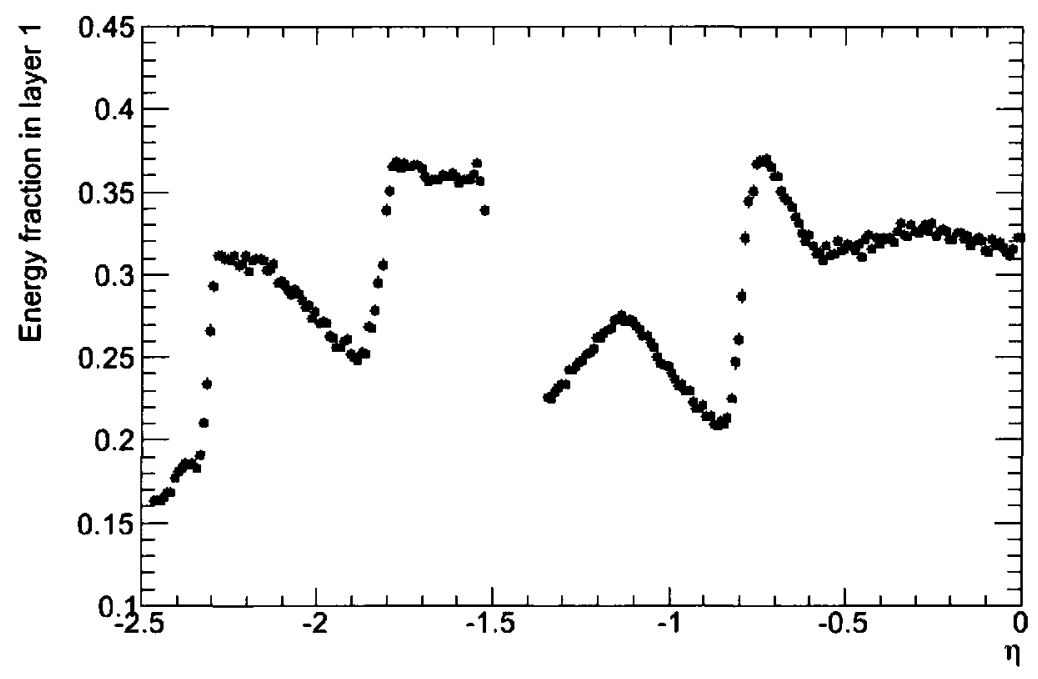

(b) S1.

FIgURE 4.15: The average nominal longitudinal energy deposition as a function of $\eta$ in each layer of the electromagnetic calorimeter. Variables are plotted for the quadrant $-\pi<\phi<-\frac{\pi}{2}(\eta<0)$, in which no extra material has been added in the Monte Carlo simulation. 
Chapter 4. Impact of Extra Material in Front of the EM Calorimeter and in the Inner Detector

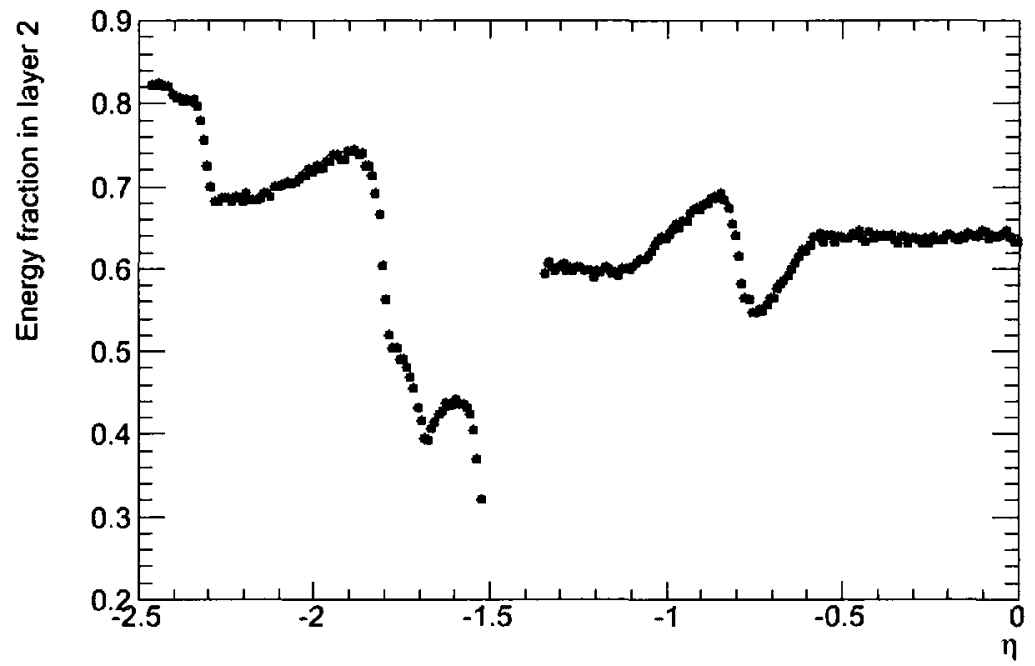

(c) S2.

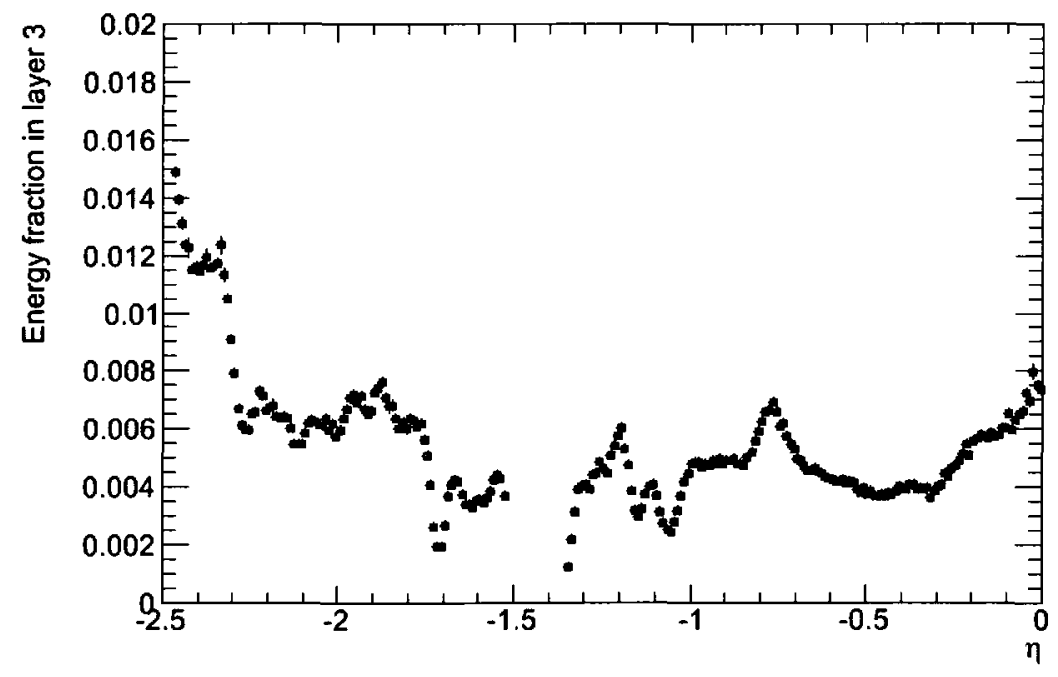

(d) S3. 
Chapter 4. Impact of Extra Material in Front of the EM Calorimeter and in the Inner Detector

coverage ends at $\eta=-1.8$, resulting in an increased energy deposit in subsequent layers. The following summarizes a detailed study of the impact of extra material on the longitudinal energy deposition in the calorimeter.

\subsubsection{Impact of Extra Material in the Cryostat}

In regions where extra material is present, one would expect to see electromagnetic showers starting earlier, compared to regions without extra material. In the ATLAS electromagnetic calorimeter, this would translate into a larger fraction of the total energy being deposited in the first layers (i.e. PS and S1) and a smaller fraction being deposited in the last layers (i.e. S2 and S3).

Figure 4.16 shows the fraction of energy deposited in each layer in both the quadrant with extra material in the cryostat and the reference quadrant (refer to Table 4.2 for $|\eta|<0.5)$. It would appear that the prediction that showers would start earlier in the distorted region has been fulfilled, especially in S1 and S2, but it is necessary to attach some significance to this result. In order to accomplish this, the estimator $R_{\text {material }}$ has been defined as

$$
R_{\text {material }}=\frac{\langle x(\text { distorted })\rangle}{\langle x(\text { reference })\rangle}
$$


Chapter 4. Impact of Extra Material in Front of the EM Calorimeter and in the Inner Detector

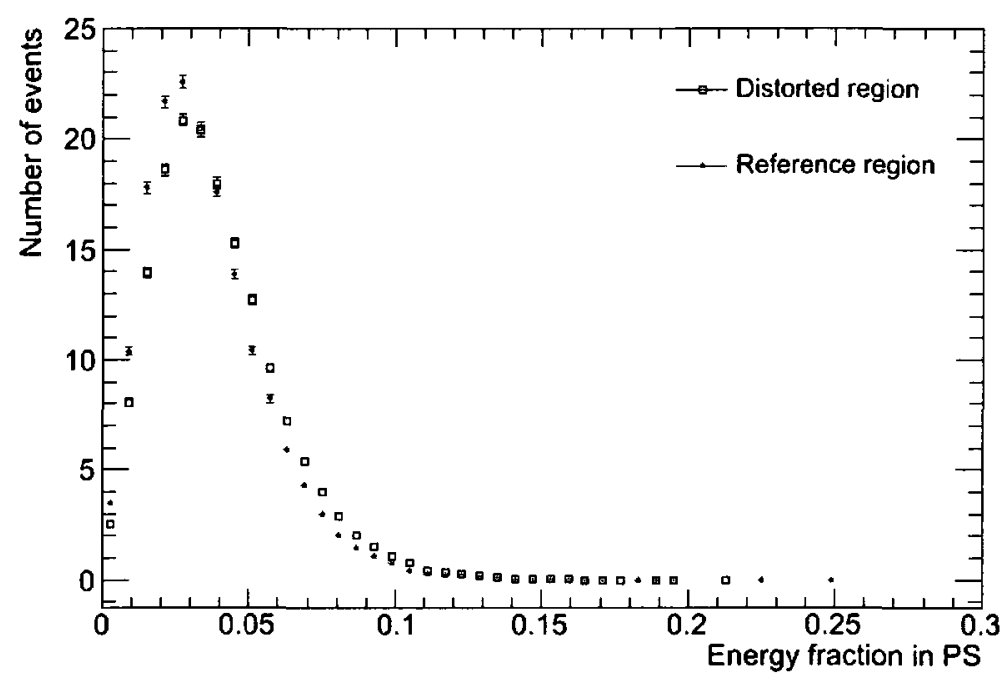

(a) PS.

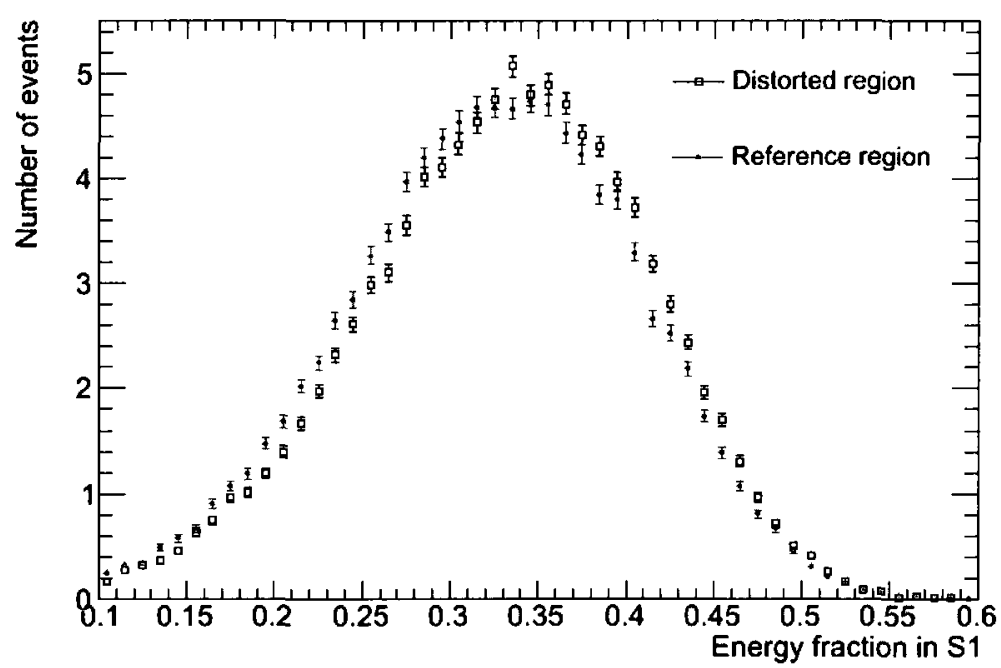

(b) S1.

FIGURE 4.16: Normalized distributions of the fraction of energy deposited in each layer of the electromagnetic barrel calorimeter in the region $|\eta|<0.5$. The distorted region corresponds to the quadrant with extra material simulated in the cryostat $\left(-\frac{\pi}{2}<\phi<0(\eta>0)\right)$, while the reference region is the quadrant $-\pi<\phi<$ $-\frac{\pi}{2} ; \eta<0$. 
Chapter 4. Impact of Extra Material in Front of the EM Calorimeter and in the Inner Detector

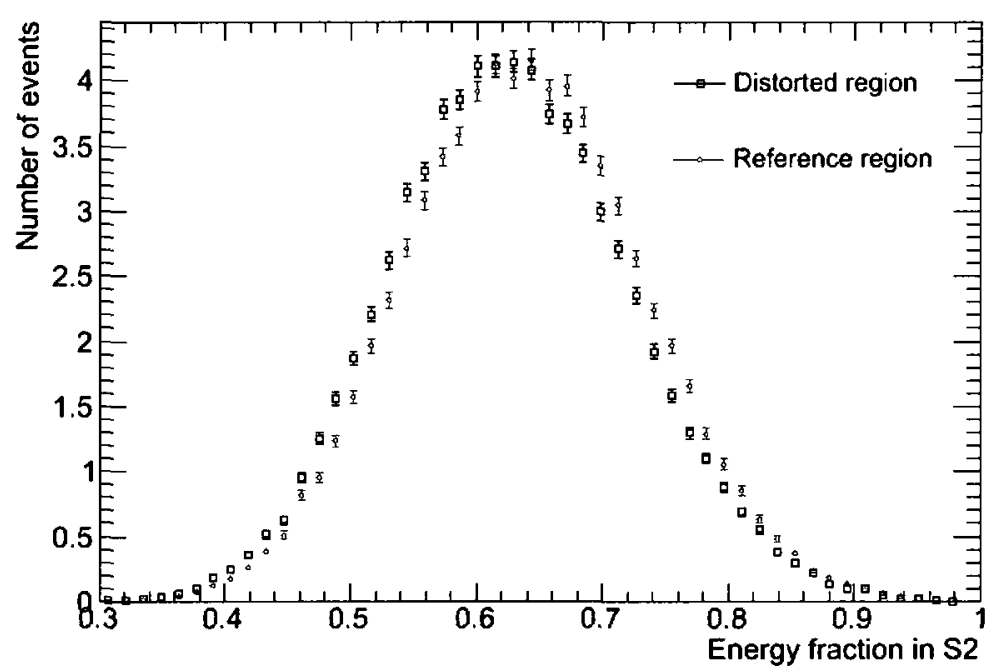

(c) S2.

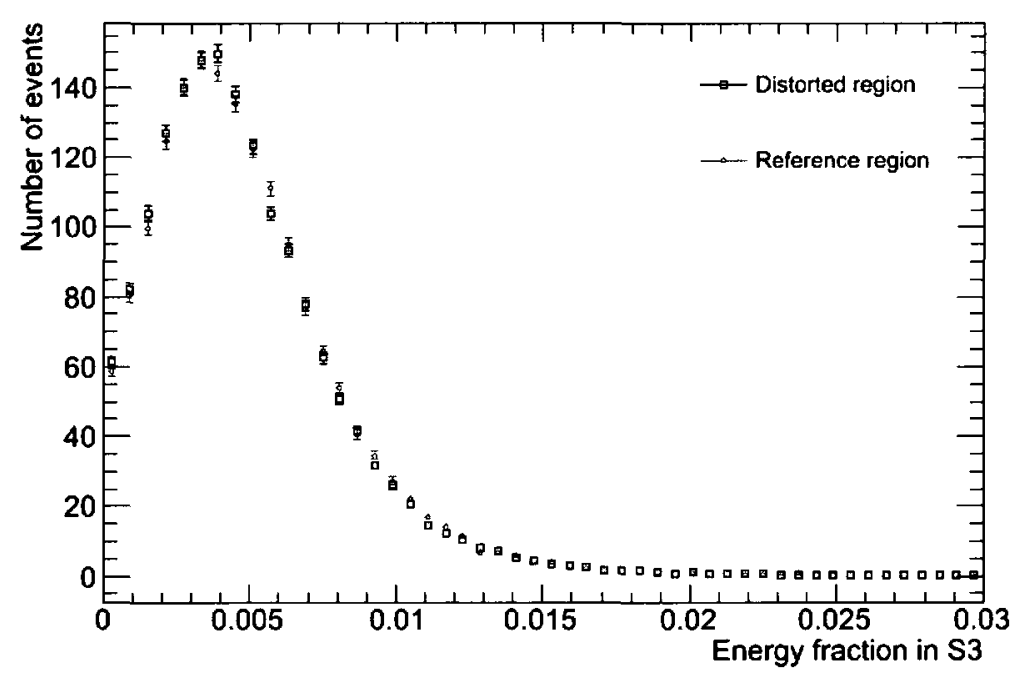

(d) S3. 
Chapter 4. Impact of Extra Material in Front of the EM Calorimeter and in the Inner Detector

where $\langle x($ distorted $)\rangle$ and $\langle x($ reference $)\rangle$ represent a given shower variable averaged over the distorted and reference regions, respectively.

When investigating the impact of extra material simulated in the cryostat on the longitudinal energy deposition, Equation 4.1 becomes (refer to Table 4.2)

$$
R_{\text {cryo }}=\frac{\left\langle x\left(-\frac{\pi}{2}<\phi<0 ; \eta>0\right)\right\rangle}{\left\langle x\left(-\pi<\phi<-\frac{\pi}{2} ; \eta<0\right)\right\rangle},
$$

where $x$ is the fraction of energy deposited in a given layer (PS, S1, S2 or S3) of the calorimeter. This estimator was calculated for each calorimeter layer in regions of $\Delta|\eta|=0.1$. The results are shown in Figure $4.17 ; R_{\text {cryo }}>1$ for the presampler and S1 and $R_{\text {cryo }}<1$ for S2 and S3. This indicates clearly, and in a statistically significant fashion, that the showers start in the extra material upstream, and so a larger fraction of energy is deposited in the PS and S1, and less energy is deposited in S2 and S3. For the presampler, the average over all points in Figure 4.17 is $\left\langle R_{\text {cryo }}\right\rangle=1.102 \pm 0.002$, which is consistent with the results of the original study [16]. Thus, of the four layers, the presampler is the most sensitive to material increases in the cryostat, although all four layers are sensitive, as demonstrated in the legend of Figure 4.17. 


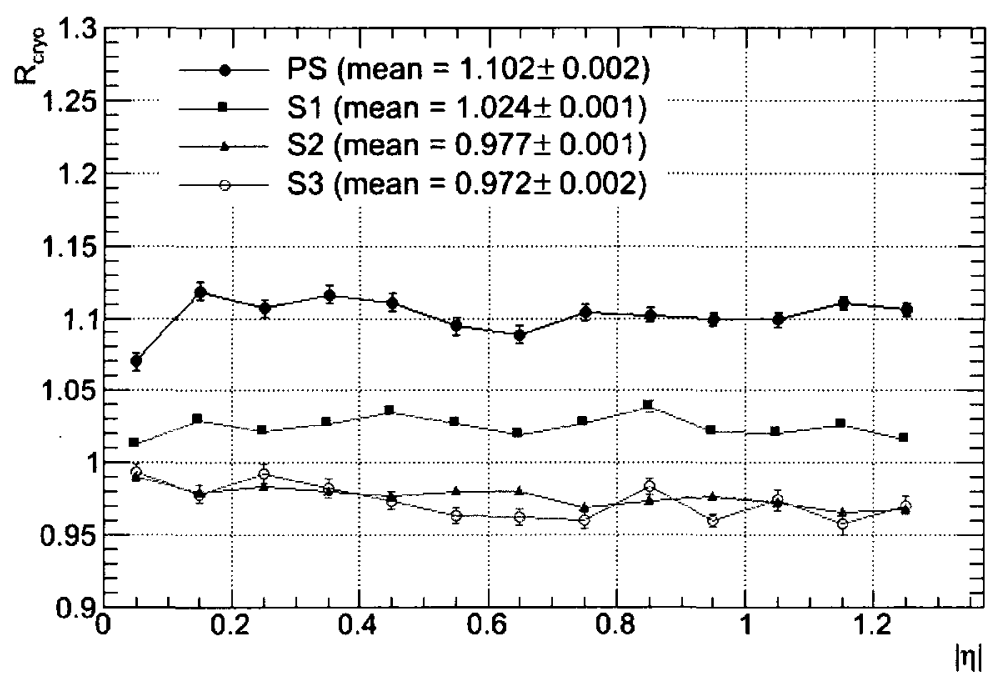

FIgure 4.17: The estimator $R_{\text {cryo }}$ as a function of $|\eta|$ for the fraction of energy deposited in each layer, calculated for 2.5 million $W \rightarrow e \nu$ events.

\subsubsection{Estimating the Sensitivity of Longitudinal Energy Loss Variables to Extra Material}

Up to this point, the purpose of this work has been mainly to replicate the results of Hubaut et al. Given that the energy deposited in each calorimeter layer has been determined to be sensitive to extra material in the cryostat, this will be further investigated and the sensitivity will be quantified as a function of the amount of extra material added. As mentioned previously, in the ideal scenario, a shower variable sensitive to material increases on the order of $1 \%$ of a radiation length would be found. The following outlines a simple "back-of-the-envelope" calculation that may be used to estimate the sensitivity of shower variables to extra material. This theoretical 
Chapter 4. Impact of Extra Material in Front of the EM Calorimeter and in the Inner Detector

calculation will first be compared to the results of the Monte Carlo simulation of 2.5 million $W \rightarrow e \nu$ events in both the reference region and the region with extra material in the cryostat (refer to Table 4.2). Then, extra material will be added to the theoretical calculation in order to determine the sensitivity of the longitudinal energy loss variables to extra material.

Recall that by integrating Equation 3.7 over a given amount of material, one obtains the amount of energy deposited over the course of an electromagnetic shower in that material. By choosing limits of integration corresponding to the boundaries of the presampler and three sampling layers of the electromagnetic calorimeter, it is possible to model the longitudinal development of a shower in each layer.

On average, a particle traversing the electromagnetic calorimeter in the region $|\eta|<0.8$ will pass through roughly $21 X_{0}$ of material, of which $82.7 \%$ is in the form of lead, $9.3 \%$ is liquid argon, $7.1 \%$ is stainless steel, and $0.9 \%$ is fibreglass/resin. In this back-of-the-envelope calculation, the stainless steel was assumed to be iron (for which the parameters in Equation 3.7 are tabulated [4]) and the fibreglass/resin was considered to be negligible enough to be treated as liquid argon. Longitudinal energy loss profiles for lead, iron and liquid argon are given in Figure 4.18. Equation 3.7 was integrated over each layer of the calorimeter (the limits of integration are based on the material distributions shown in Figure 3.8 and are given in Table 4.3) for lead, iron and liquid argon individually, using initial electron energy values ranging from 20 
Chapter 4. Impact of Extra Material in Front of the EM Calorimeter and in the Inner Detector

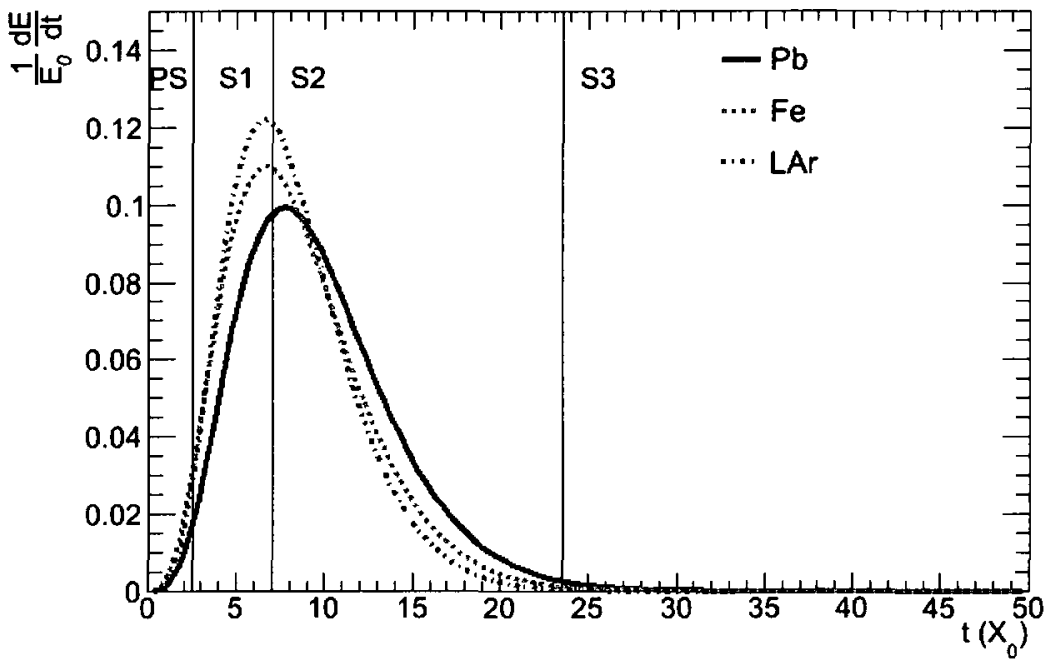

FIgURE 4.18: Longitudinal energy loss profiles for a $35 \mathrm{GeV}$ electron in lead, iron and liquid argon. The vertical lines represent the boundaries between the PS, S1, S2 and S3.

$\mathrm{GeV}-250 \mathrm{GeV}$. This energy range is similar to that of the electrons in the $W \rightarrow e \nu$ Monte Carlo sample, as shown in Figure 4.9. The results of the integration were then weighted based on the proportions of each material in the calorimeter and averaged to yield the amount of energy deposited in a mixture of the calorimeter materials. This served to further simplify the calculation by approximating the alternating accordion structure of the calorimeter as a homogeneous system.

The estimated energy loss obtained with this method (expressed as a fraction of the initial electron energy) is shown in Figure 4.19 as a function of the initial energy. For comparison purposes, the Monte Carlo energy fraction variables for the $W \rightarrow e \nu$ sample are also shown in increments of $\Delta E=10 \mathrm{GeV}$. For the purposes of this 
Chapter 4. Impact of Extra Material in Front of the EM Calorimeter and in the

\begin{tabular}{l||c|r} 
Layer & Lower bound $\left(X_{0}\right)$ & Upper bound $\left(X_{0}\right)$ \\
\hline \hline PS \& upstream & 0 & 2.5 \\
\hline S1 & 2.5 & 7 \\
\hline S2 & 7 & 23.5 \\
\hline S3 \& beyond & 23.5 & 50
\end{tabular}

TABLE 4.3: Limits of integration over each layer of the electromagnetic barrel calorimeter. The presampler and third layer contain approximate corrections to account for losses upstream and downstream, respectively.

analysis, only the region $|\eta|<0.5$ was included, due to its relative "flatness" in terms of material content, as shown in the top and bottom left-hand plots in Figure 3.8.

In Figure 4.19, the "theoretical" mixture material calculation gives quite good agreement with the full $W \rightarrow e \nu$ Monte Carlo simulation of the energy deposited in the reference region of the calorimeter for layers 1 and 2 - the layers in which the overwhelming majority of the shower energy is deposited. In the presampler and S3, on the other hand, the agreement is not so good, but this was to be expected. In both cases, this simple calculation does not take fully into account the actual composition of the material upstream (the cryostat wall and the inner detector components), nor does it accurately describe the downstream leakage. In addition, the calculation does not properly take into account the composition of the presampler, which is almost $100 \%$ liquid argon. All of these factors are treated correctly in the official ATLAS Monte Carlo used in Figure 4.19. It is worthwhile to remember that this method is not meant to be a precise replacement for the existing simulations, but merely a quick 
Chapter 4. Impact of Extra Material in Front of the EM Calorimeter and in the

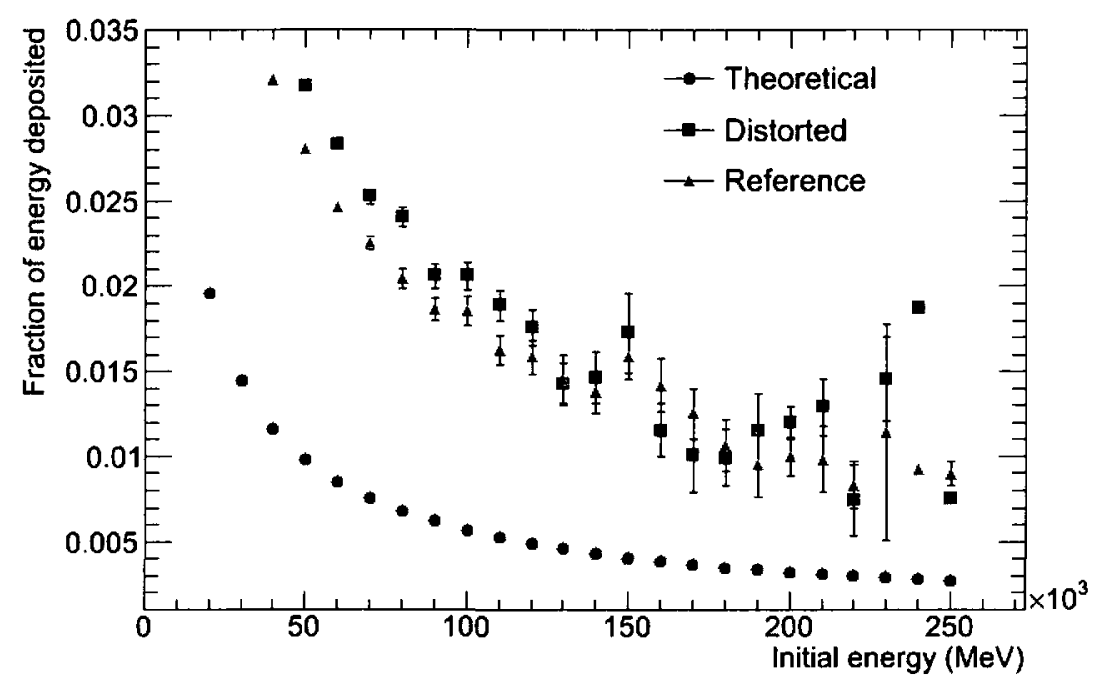

(a) PS.

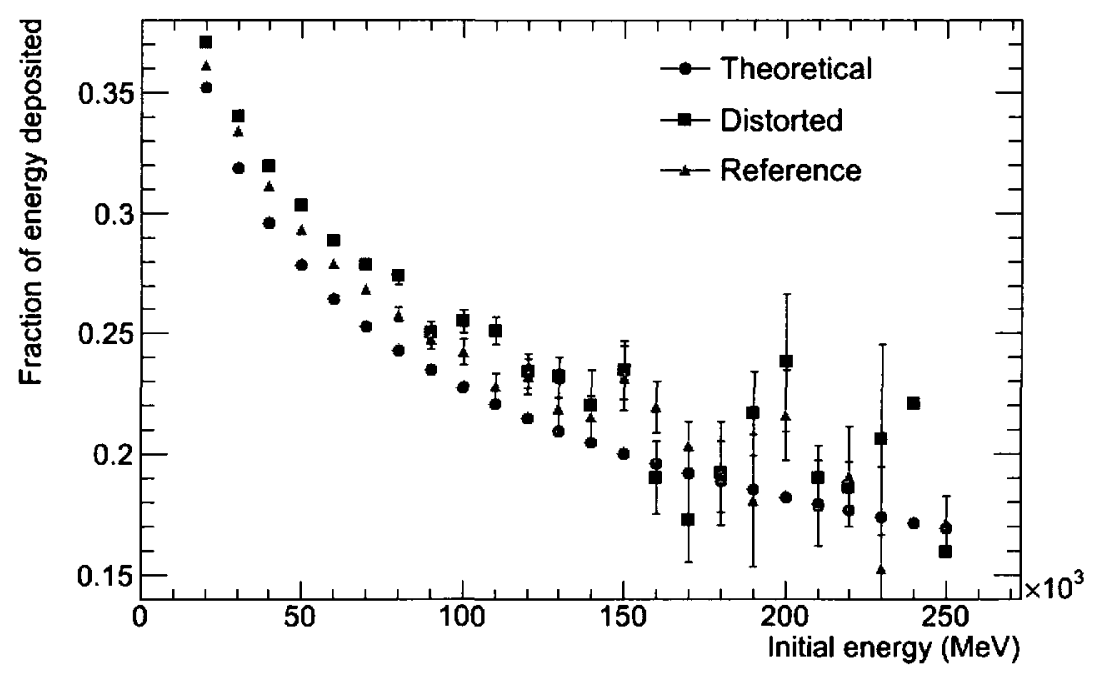

(b) $\mathrm{S} 1$.

FIGURE 4.19: Fraction of energy deposited in each layer of the calorimeter as a function of initial electron energy. The theoretical curve is a weighted average of curves calculated for lead, iron and liquid argon. The distorted and reference curves correspond to the quadrants with extra material in the cryostat and with no extra material, respectively, in the simulated CSC-01-02-00 geometry. 
Chapter 4. Impact of Extra Material in Front of the EM Calorimeter and in the Inner Detector

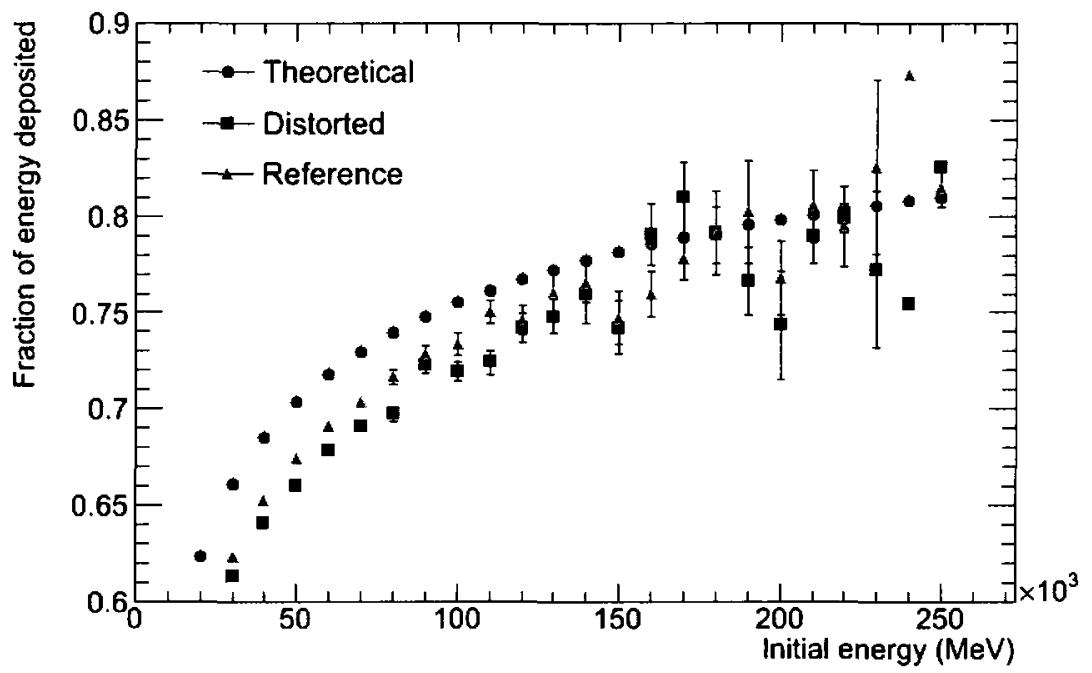

(c) $\mathrm{S} 2$.

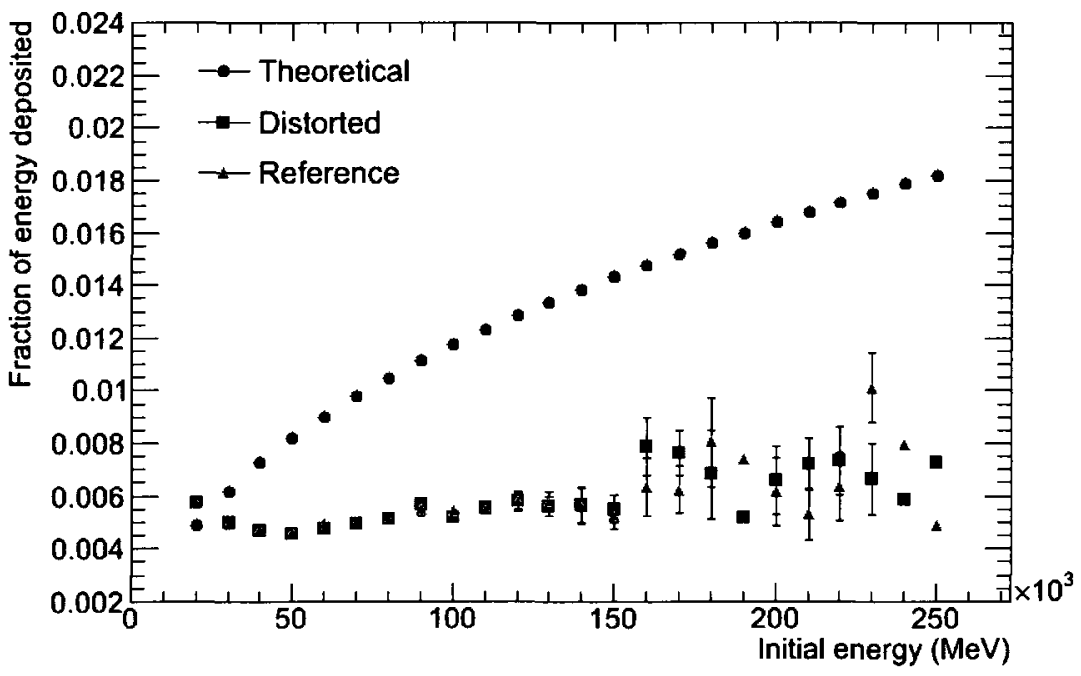

(d) $\mathrm{s} 3$. 
Chapter 4. Impact of Extra Material in Front of the EM Calorimeter and in the Inner Detector

and simple means of facilitating an estimate of the sensitivity of the shower variables to extra material. Hence, only the first and second calorimeter layers were considered from this point onward.

To determine how the shower energy deposition would respond to extra material added in front of the calorimeter, the integration of Equation 3.7 was repeated, but with the limits of integration adjusted to reflect the presence of extra material in the amount of $0.01 X_{0}, 0.05 X_{0}, 0.1 X_{0}$ and $0.25 X_{0}$ located before the presampler. The resulting fractional energy deposition values were then divided by the original values (shown in Figure 4.19), and the ratios were plotted as a function of initial electron energy (up to $150 \mathrm{GeV}$ ) in Figure 4.20. The uncertainties were derived using the full ATLAS Monte Carlo statistics from Figure 4.19. Note that the results shown in Figure 4.20 follow the same trend as the full Monte Carlo sample; the ratios are greater than unity in S1 and less than unity in S2. Based on the statistics of 2.5 million events, we can expect the fractional energy loss variables to be sensitive to amounts of material on the order of $1 \%-5 \%$ of a radiation length at energies of up to $80 \mathrm{GeV}$, which corresponds to the approximate level of precision required for optimum performance. These results agree with the conclusion of Hubaut et al., which was obtained using a different method. 


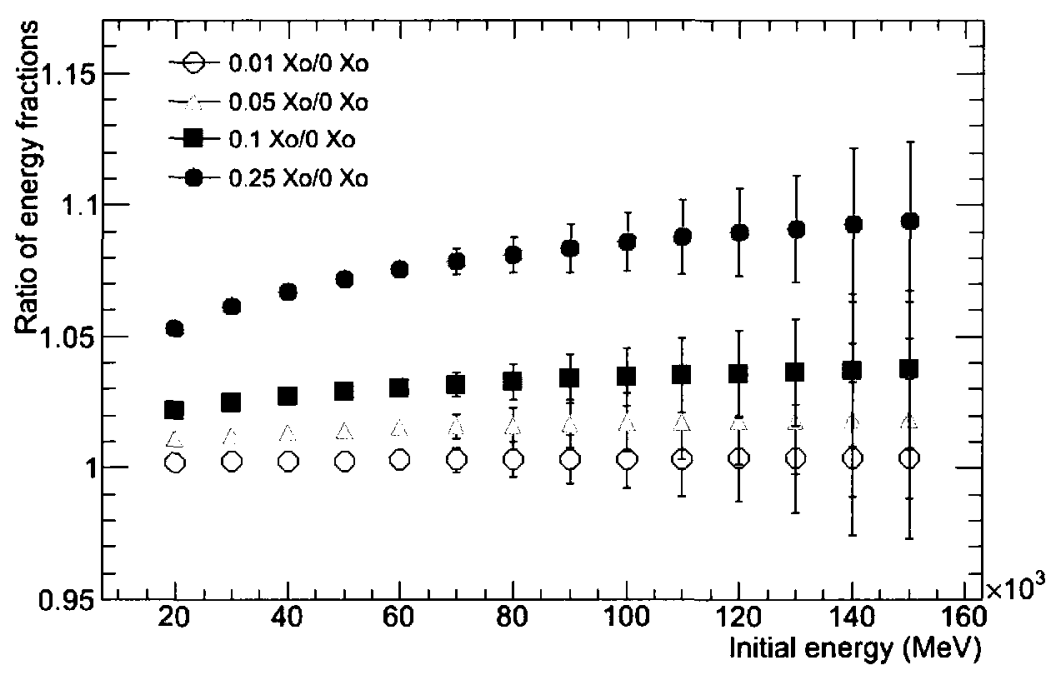

(e) S1.

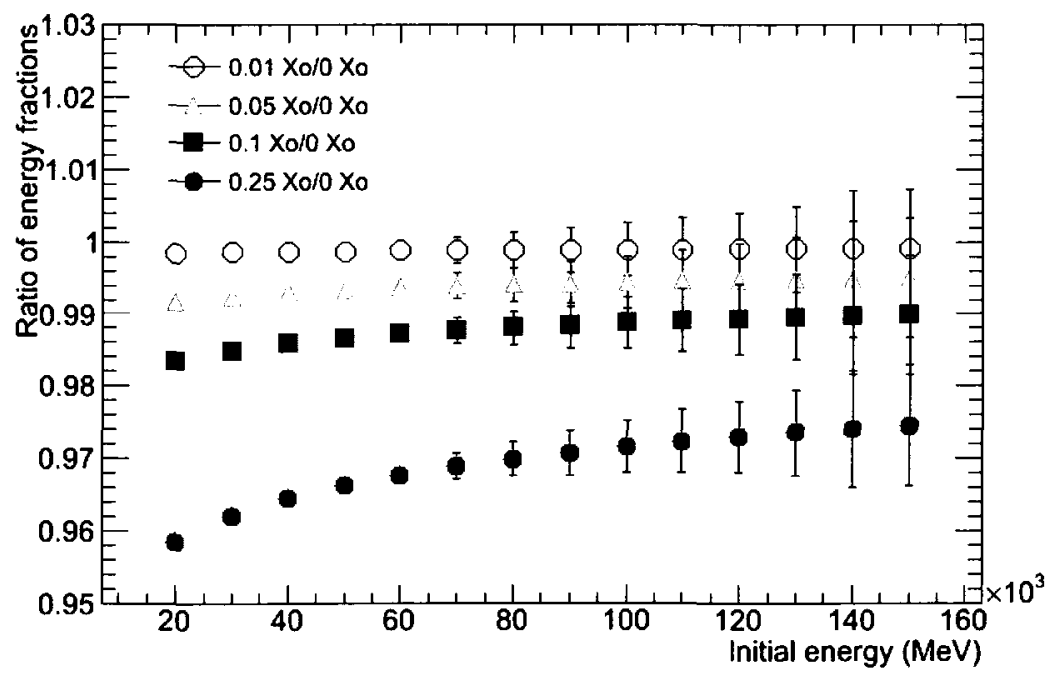

(f) S2.

FiguRE 4.20: Ratio of fractional energy deposition with extra material added in front of the calorimeter to that without extra material, as a function of initial electron energy for S1 and S2. 


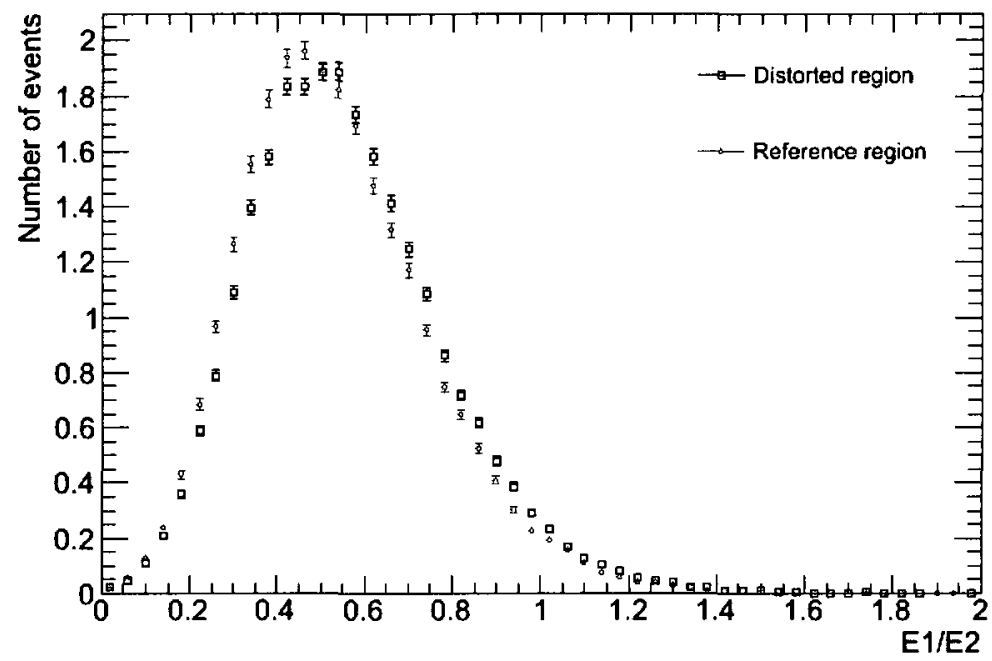

FIgURE 4.21: The normalized distribution of the new shower variable, $E_{1} / E_{2}$. The distorted region corresponds to the quadrant with extra material in the cryostat, and the reference region corresponds to the quadrant with no extra material, as defined in Table 4.2 .

\subsubsection{Attempt to Find a New Sensitive Shower Variable}

The next step in the analysis was an attempt to find a new shower variable having an even higher sensitivity and robustness by combining the individual layer variables. The PS and S3 contain correction factors, which must be confirmed, and so an ideal shower variable would have fewer corrections in order to minimize systematic errors and maximize robustness. Since the majority of the shower energy is deposited in the first and second layer and since the performance of these two layers is well understood, 
Chapter 4. Impact of Extra Material in Front of the EM Calorimeter and in the Inner Detector

these two variables were deemed to be the most useful. The ratio $E_{1} / E_{2}$, defined as

$$
\frac{E_{1}}{E_{2}} \equiv \frac{\text { Fraction of energy deposited in the first layer }}{\text { Fraction of energy deposited in the second layer }}
$$

was chosen, with the hope that it would be more robust than any of the individual energy loss variables. The distribution of $E_{1} / E_{2}$ in the distorted (cryostat) and reference regions is given in Figure 4.21. The estimator $R_{\text {cryo }}$ (defined in Equation 4.2) was calculated as before for this new variable; the result is shown in Figure 4.22 as a function of $|\eta|$ and as a function of the amount of material added. A linear fit, shown as a solid line, gives $R_{\text {cryo }}=(0.17 \pm 0.04) \cdot \Delta t_{\text {mat }}+(1.027 \pm 0.005)$ (statistical errors only), where $\Delta t_{m a t}$ is the change in the thickness of material in the cryostat, in units of $X_{0}$. A second linear fit, shown as a dashed line, was forced to pass through the point $(0,1)$ to give a rough idea of the systematics associated with the fit (since, in theory, $R_{\text {cryo }}$ should be equal to 1 in the absence of extra material in one region). This fit yields the relation $R_{\text {cryo }}=(0.364 \pm 0.009) \cdot \Delta t_{\text {mat }}+1$.

In order to compare the sensitivity of $E_{1} / E_{2}$ with the individual layer variables, the statistical significance $S_{c r y o}$ was calculated using the relation

$$
S_{\text {cryo }}=\frac{\left|R_{\text {cryo }}-1\right|}{\sigma_{R}^{\text {stat }}},
$$

where $\sigma_{R}^{\text {stat }}$ is the statistical error on $R_{\text {cryo. }}$ As shown in Figure 4.23, the fraction 


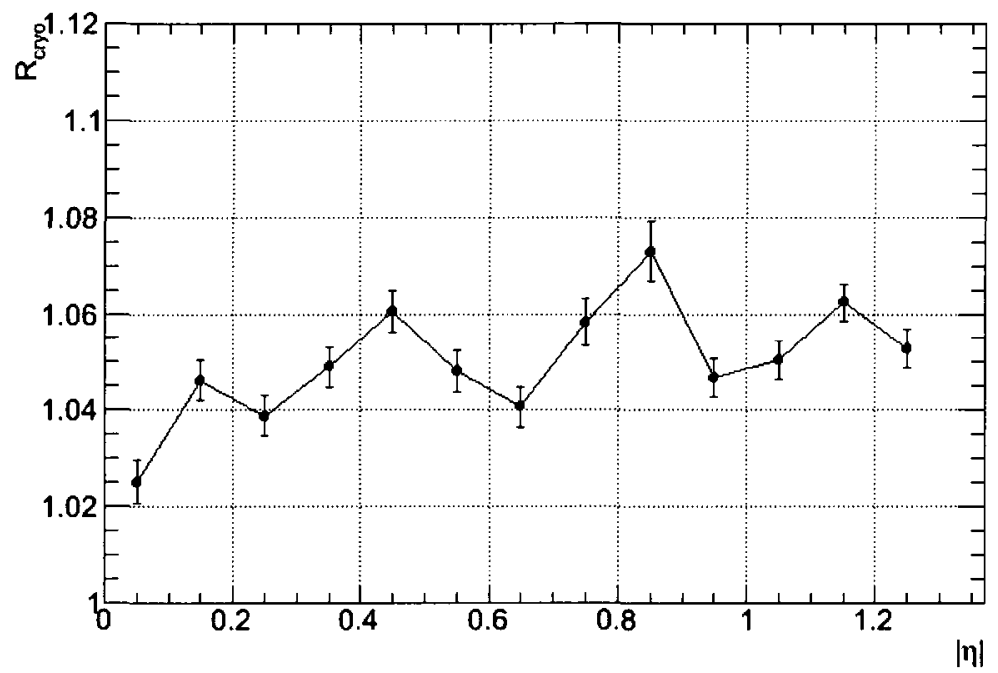

(a) $R_{\text {cryo }}$ as a function of $|\eta|$ for $E_{1} / E_{2}$.

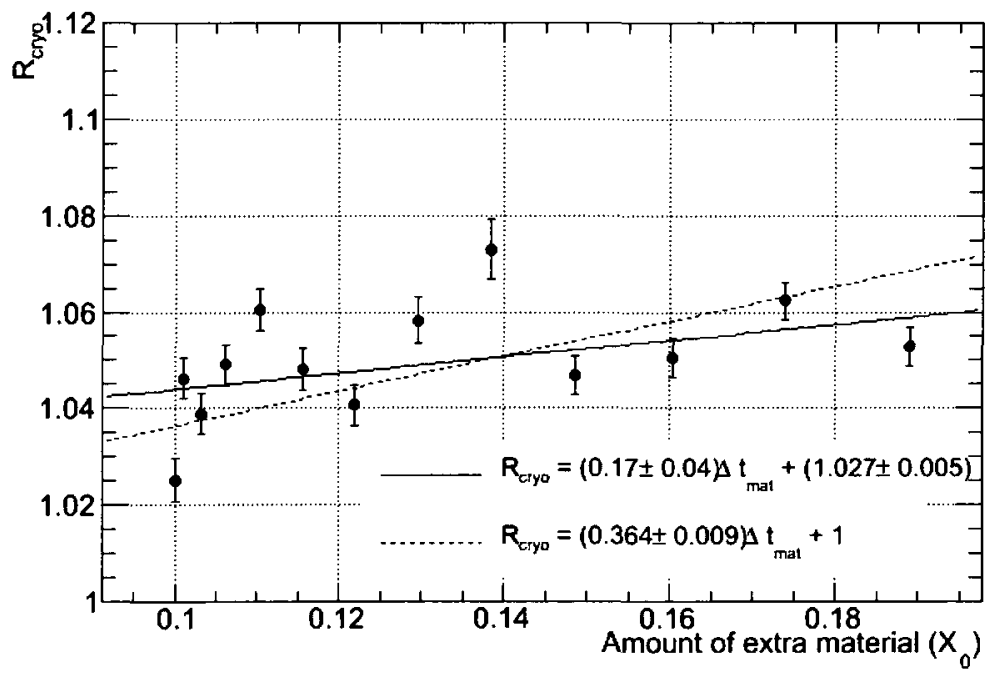

(b) $R_{\text {cryo }}$ as a function of material added for $E_{1} / E_{2}$.

FiguRE 4.22: (a) The estimator $R_{\text {cryo }}$ as a function of $|\eta|$ for $E_{1} / E_{2}$, calculated for 2.5 million $W \rightarrow e \nu$ events. $\left\langle R_{\text {cryo }}\right\rangle=1.049 \pm 0.001$. (b) $R_{\text {cryo }}$ as a function of the amount of material added. The solid line is the best fit to the data and the dashed line is a fit forced to pass through the point $(0,1)$. 
Chapter 4. Impact of Extra Material in Front of the EM Calorimeter and in the Inner Detector

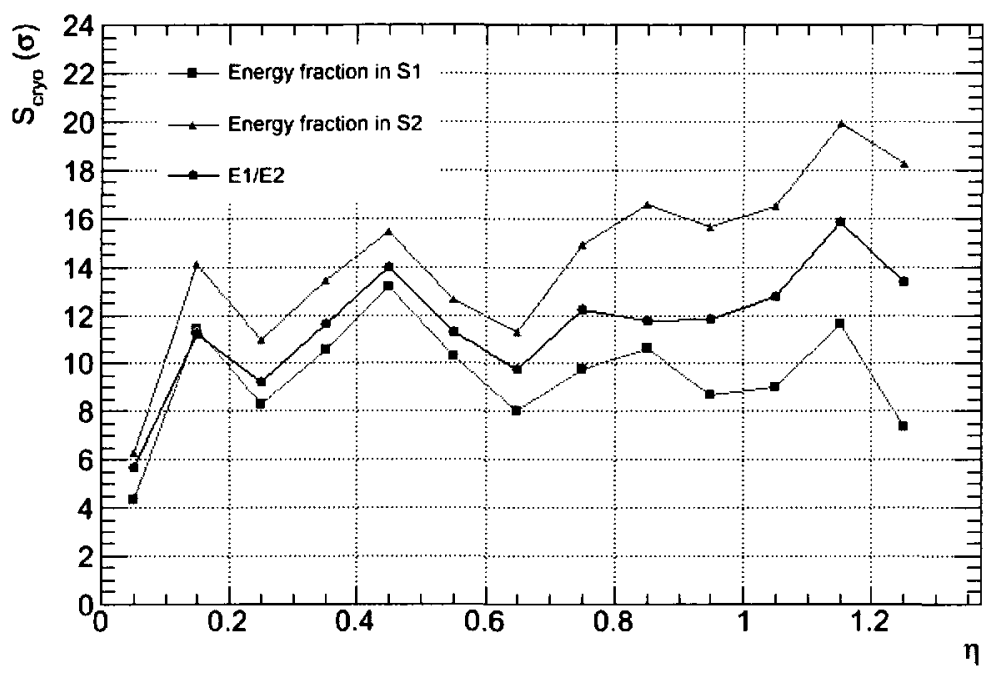

FIgURE 4.23: Statistical significance of the $R_{\text {cryo }}$ estimators as a function of $\eta$, obtained using Equation 4.23.

of energy in $\mathrm{S} 2$ has the highest overall statistical significance, while $E_{1} / E_{2}$ has a slightly lower statistical significance and the fraction of energy in S1 has the lowest significance. Nonetheless, $E_{1} / E_{2}$ is still highly sensitive to extra material and, as such, is a valid discriminating variable for the purpose of this study. $E_{1} / E_{2}$ is less sensitive to the overall energy scale, and also provides information about the shower shape development, unlike the individual layer variables; therefore, it is a more robust variable.

Once the validity of $E_{1} / E_{2}$ as a sensitive variable was established, the backof-the-envelope theoretical calculation was once again employed in order to quantify this variable's sensitivity to extra material. As before, Equation 3.7 was integrated 
Chapter 4. Impact of Extra Material in Front of the EM Calorimeter and in the Inner Detector

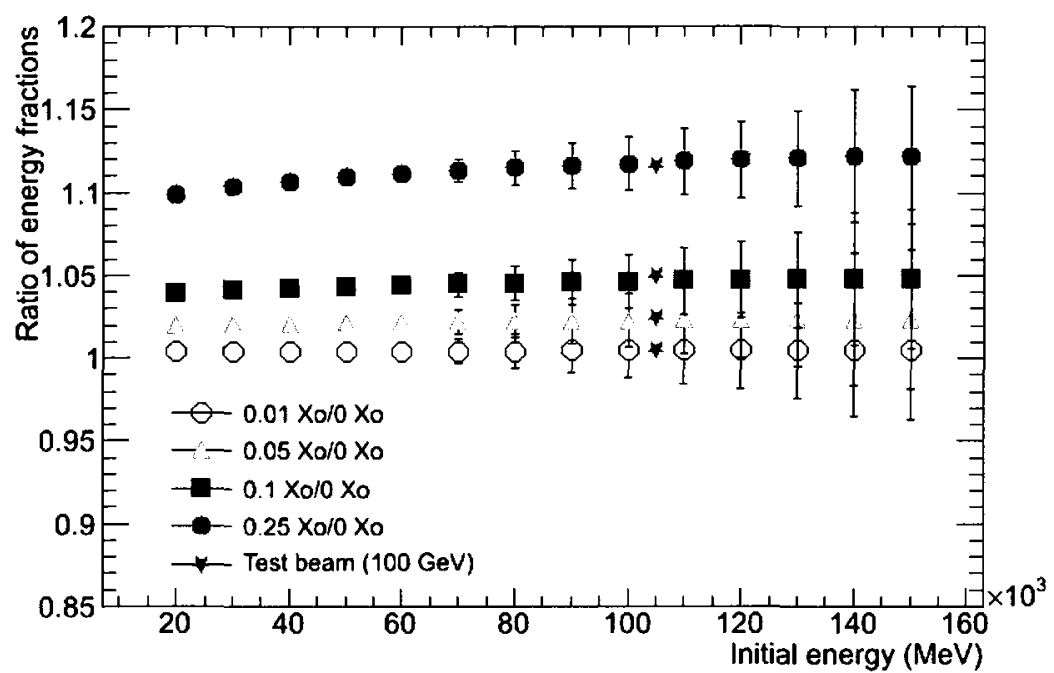

FIgURE 4.24: Ratio of $E_{1} / E_{2}$ with extra material added in front of the calorimeter to that without extra material, as a function of initial electron energy. "Test beam" points (pink stars) corresponding to an initial energy of $100 \mathrm{GeV}$ (offset by $5 \mathrm{GeV}$ for clarity) were calculated using the results from the 2004 combined test beam.

with $0.01 X_{0}, 0.05 X_{0}, 0.1 X_{0}$ and $0.25 X_{0}$ added in front of the calorimeter. $E_{1} / E_{2}$ was calculated, and the ratio of the new variable with extra material to that without extra material was taken. The resulting ratios (as a function of the initial electron energy) are shown in Figure 4.24, with uncertainties derived using the full ATLAS Monte Carlo statistics. This new variable is sensitive to material increases on the order of $1 \%-5 \%$ of a radiation length, up to energies of $80 \mathrm{GeV}$.

Results from the 2004 combined test beam [36] confirm the dependence of $E_{1} / E_{2}$ on the amount of extra material. During this test, an electromagnetic barrel calorimeter module, identical to those currently installed in ATLAS, was placed in 
Chapter 4. Impact of Extra Material in Front of the EM Calorimeter and in the Inner Detector

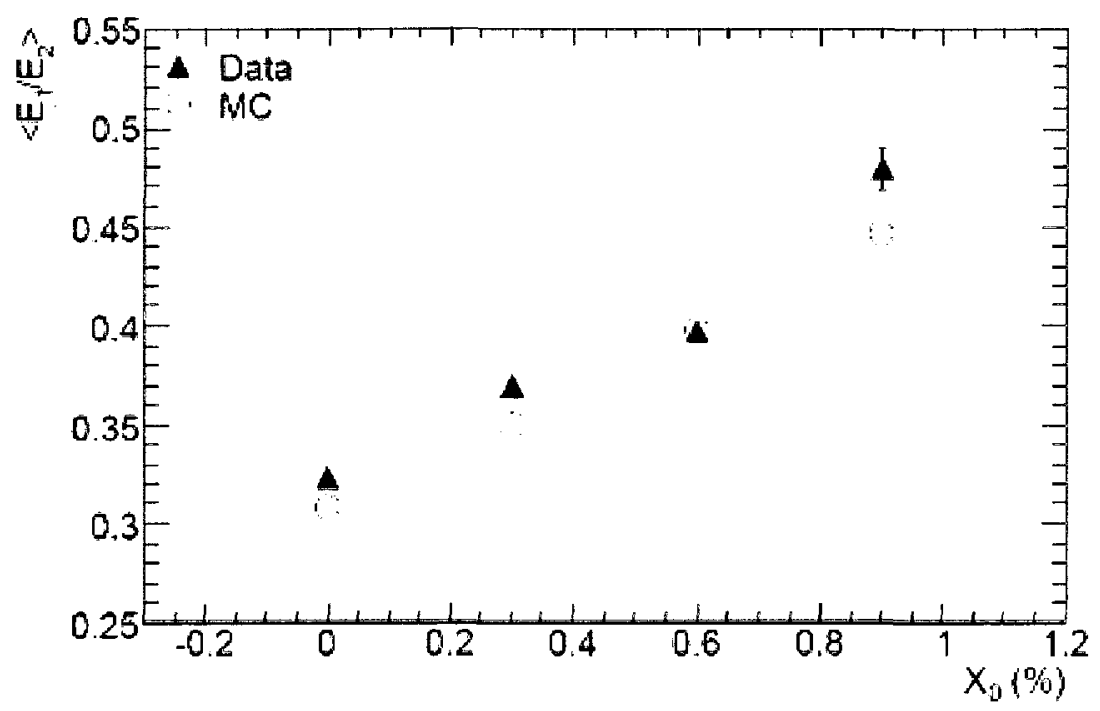

FIgure 4.25: Results of the 2004 combined test beam for $\left\langle E_{1} / E_{2}\right\rangle$ as a function of the amount of extra material. Note that the units of the horizontal axis should be expressed as a fraction, rather than as a percentage. Figure from [36].

the H8 beam line of the CERN SPS accelerator along with modules from the other ATLAS subdetectors in a configuration as similar as possible to the design geometry of the full detector. Up to three plates of aluminum, each having a thickness of $0.3 X_{0}$, were placed in front of the calorimeter, and the effect of this extra material on $E_{1} / E_{2}$ was determined for beams of electrons with energies of $9 \mathrm{GeV}-180 \mathrm{GeV}$.

Figure 4.25 illustrates this relationship for $100 \mathrm{GeV}$ electrons; it can be expressed as $\Delta\left(E_{1} / E_{2}\right)=0.154 \cdot \Delta t_{m a t}[38]$, where $\Delta t_{m a t}$ is the difference in material thickness, measured in radiation lengths $\left(X_{0}\right)$. Note that this value lies within the error on the slope of the fit in Figure 4.22 (b). This relationship was used to calculate $E_{1} / E_{2}$ values with $0.01 X_{0}, 0.05 X_{0}, 0.1 X_{0}$ and $0.25 X_{0}$ of material added in front 
Chapter 4. Impact of Extra Material in Front of the EM Calorimeter and in the Inner Detector

of the calorimeter, and the resulting data points are shown as pink stars (offset by $5 \mathrm{GeV}$ for clarity) in Figure 4.24. It is apparent from the test beam results that this back-of-the-envelope calculation yields a valid approximation of the longitudinal development of electromagnetic showers in the calorimeter.

\subsubsection{Effect of Extra Material Between the Presampler and the First Sampling Layer}

The sensitivity of the new variable, $E_{1} / E_{2}$, to extra material between the PS and S1 was also investigated. To do so, the ratio $R_{P S-S 1}$ was calculated, using the specific definition of Equation 4.1 as given by Table 4.2:

$$
R_{P S-S 1}=\frac{\left\langle E_{1} / E_{2}\left(-\frac{\pi}{2}<\phi<0 ; \eta<0\right)\right\rangle}{\left\langle E_{1} / E_{2}\left(-\pi<\phi<-\frac{\pi}{2} ; \eta<0\right)\right\rangle} .
$$

Figure 4.26 illustrates the results of this calculation. As $|\eta|$ increases, so does $R_{P S-S 1}$, indicating that $E_{1} / E_{2}$ is sensitive to the extra material. The extra material in front of the first layer causes the showers to start earlier, resulting in a larger fraction of energy being deposited in S1 and a smaller fraction being deposited in S2. The solid line in Figure 4.26 (b) represents the best fit to the data, while the dashed line represents a fit forced to pass through the point $(0,1)$. For this particular variable, the two fit results are in agreement, within the statistical error range. 


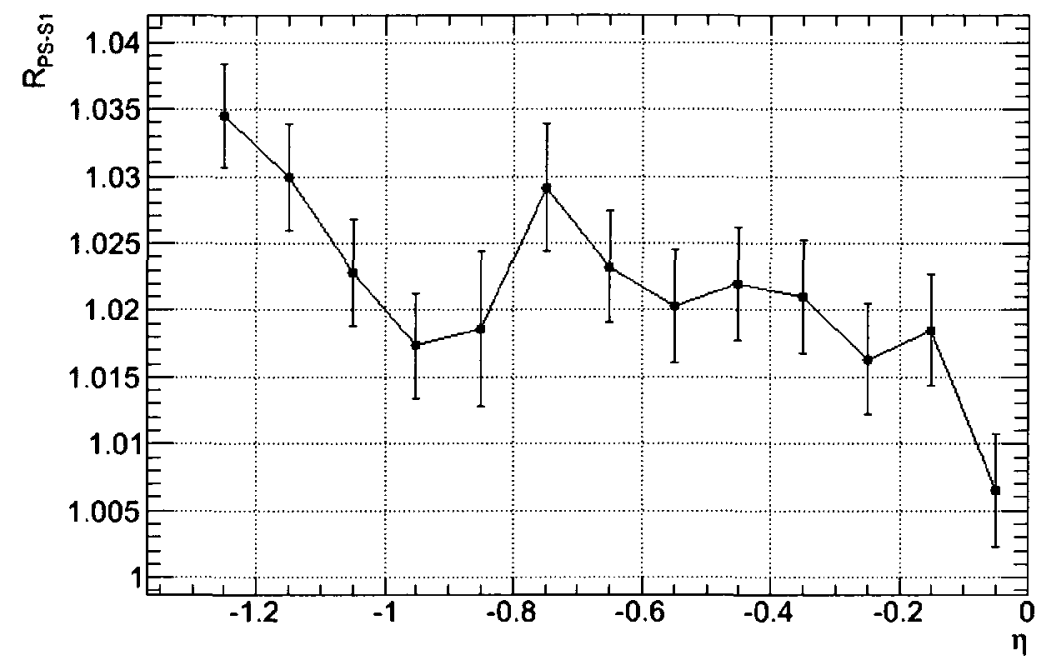

(a) $R_{P S-S 1}$ as a function of $\eta$.

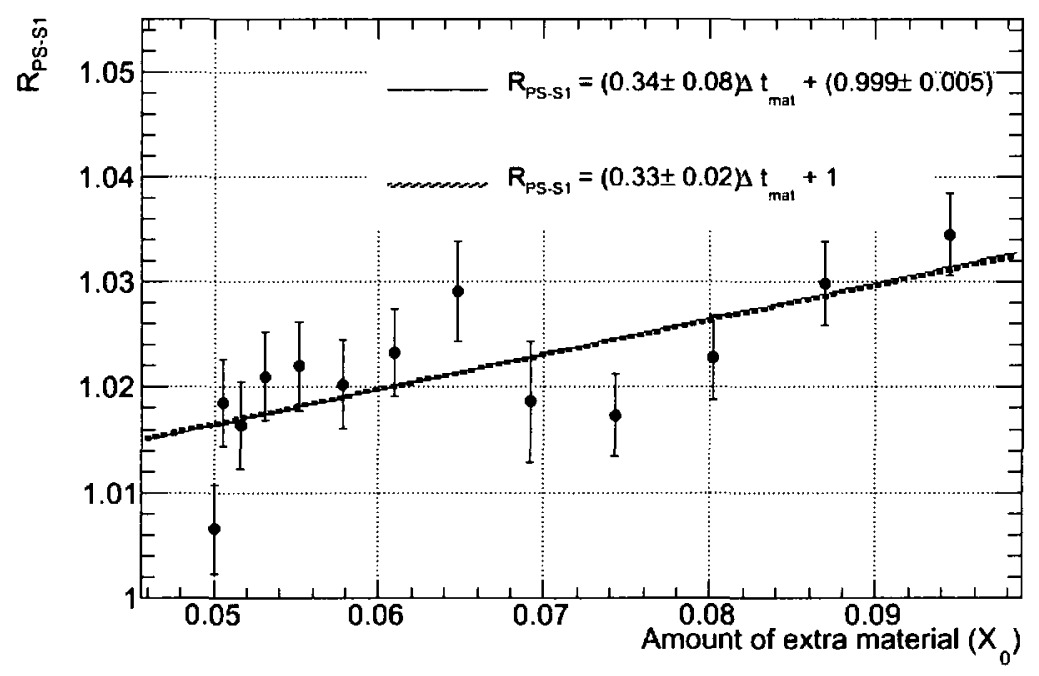

(b) $R_{P S-S 1}$ as a function of material added.

FIgURE 4.26: $R_{P S-S 1}$ for the new sensitive variable, $E_{1} / E_{2}$, as a function of $\eta$ (a) and as a function of the amount of material added (b). The solid line in (b) is the best fit to the data, while the dashed line represents a fit forced to pass through the point $(0,1)$. 
Chapter 4. Impact of Extra Material in Front of the EM Calorimeter and in the Inner Detector

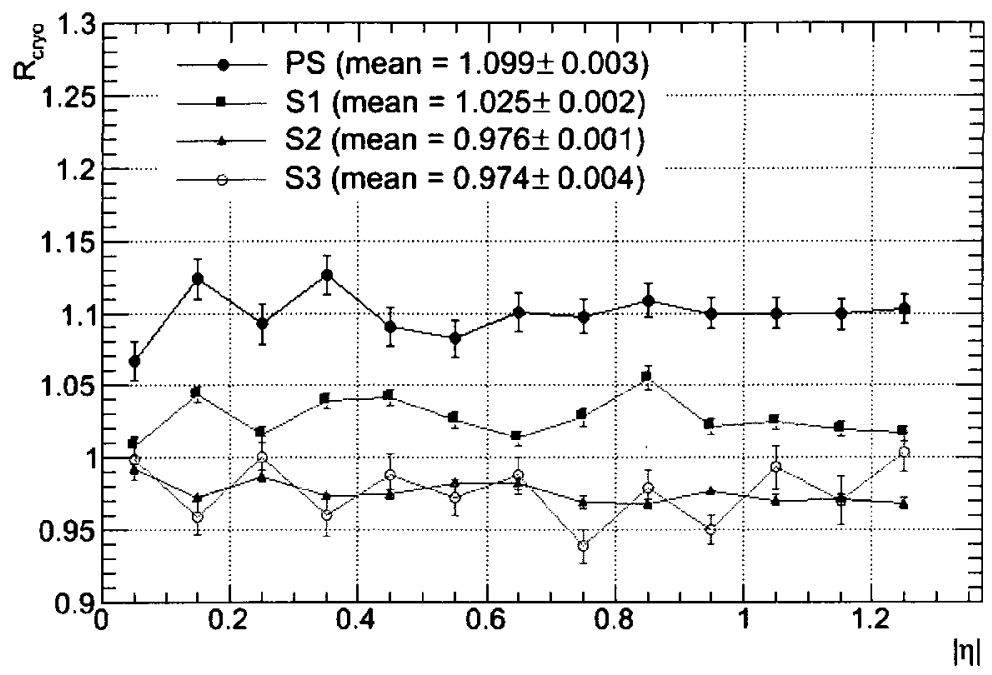

FIGURE 4.27: $R_{\text {cryo }}$ for the energy deposited in each calorimeter layer with 500,000 events.

\subsubsection{Feasibility With Early Data}

In order to have 2.5 million $W \rightarrow e \nu$ events after trigger and acceptance cuts (at least one lepton with $|\eta|<2.7$ and $p_{T}>10 \mathrm{GeV}$ ), resulting in 1.5 million events after electron identification, we would require roughly $475 \mathrm{pb}^{-1}$ of data produced at $10 \mathrm{TeV}$. During the 2009-2010 run period, the LHC is expected to produce roughly $200 \mathrm{pb}^{-1}$ at $10 \mathrm{TeV}$. Thus, in order to determine the feasibility of performing this study with early data, $R_{\text {cryo }}$ was calculated for smaller samples of the Monte Carlo events.

With sample sizes of 1,000,000 events (not shown) and 500,000 events (shown in Figure 4.27 and Figure 4.28), the sensitivity to extra material was still apparent, 
Chapter 4. Impact of Extra Material in Front of the EM Calorimeter and in the Inner Detector

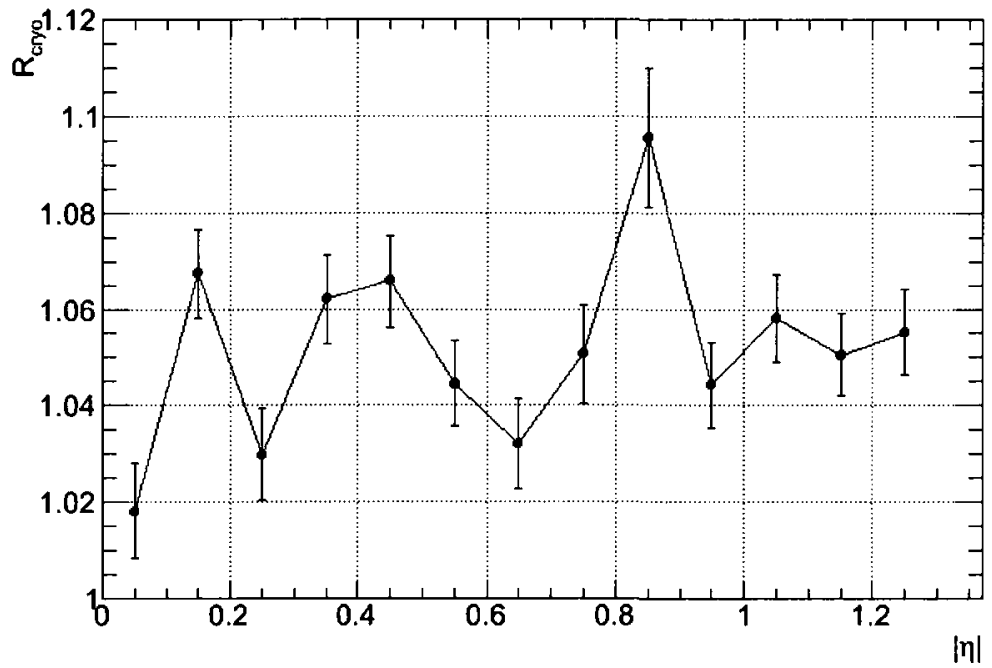

(a) $R_{\text {cryo }}$ for $E_{1} / E_{2}$ as a function of $|\eta|$.

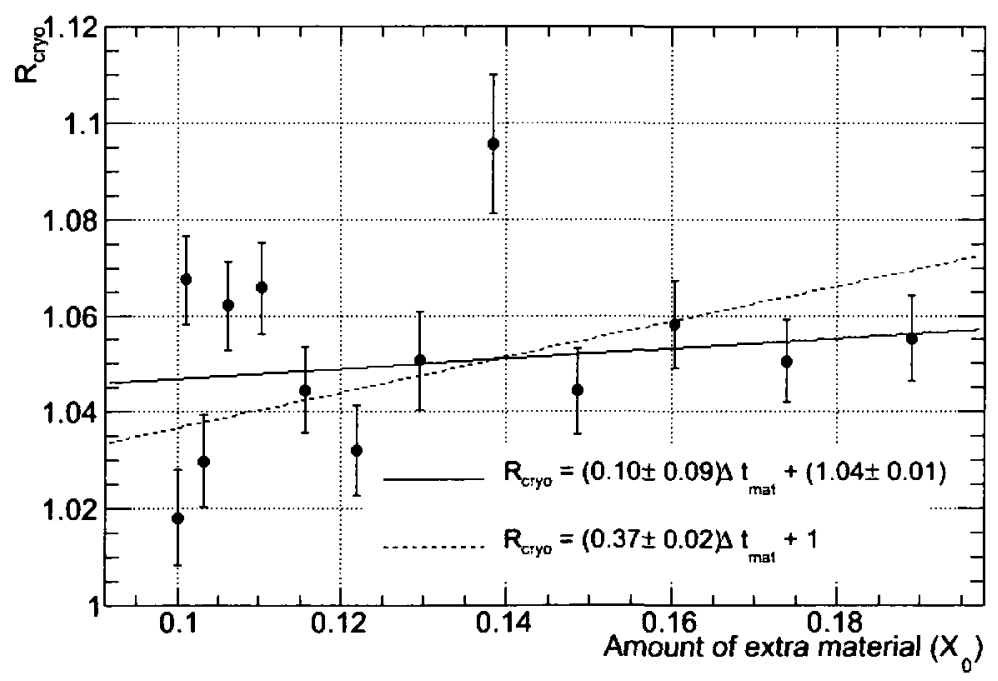

(b) $R_{\text {cryo }}$ for $E_{1} / E_{2}$ as a function of added material.

FiguRE $4.28: R_{\text {cryo }}$ for $E_{1} / E_{2}$ with 500,000 events. 
Chapter 4. Impact of Extra Material in Front of the EM Calorimeter and in the Inner Detector

although to a lesser extent, as can be shown by comparing the slopes of the fits in Figure 4.28 (b) and Figure 4.22 (b). As before, the best fit to the data in Figure 4.28 (b) is given by a solid line, and the fit given by a dashed line was forced

to pass through the point $(0,1)$. A much smaller sample size $(50,000$ events) was also tested; at this level, statistical fluctuations overshadowed any sensitivity. Based on this reasoning, approximately 500,000 events, corresponding to roughly $160 \mathrm{pb}^{-1}$ of raw data at $10 \mathrm{TeV}$, would be sufficient to be sensitive to the amount of extra material added in this Monte Carlo simulation.

\subsection{Impact of Bremsstrahlung in the Inner Detec-} tor

Bremsstrahlung events can be used to "x-ray" the inner detector. In regions of the detector where more material is present, more bremsstrahlung events occur. By plotting the truth $R-\phi$ coordinates ( $R$ is the radial coordinate defined in Equation 2.2) of the bremsstrahlung events in the Monte Carlo sample, a fairly clear image of the inner detector may be produced, as illustrated in Figure 4.29. In this image, the relatively dense pixel and SCT layers and the TRT show up as darker regions, indicating that a greater number of bremsstrahlung events occur in these structures than in the less dense surrounding regions. By correlating bremsstrahlung truth information to 


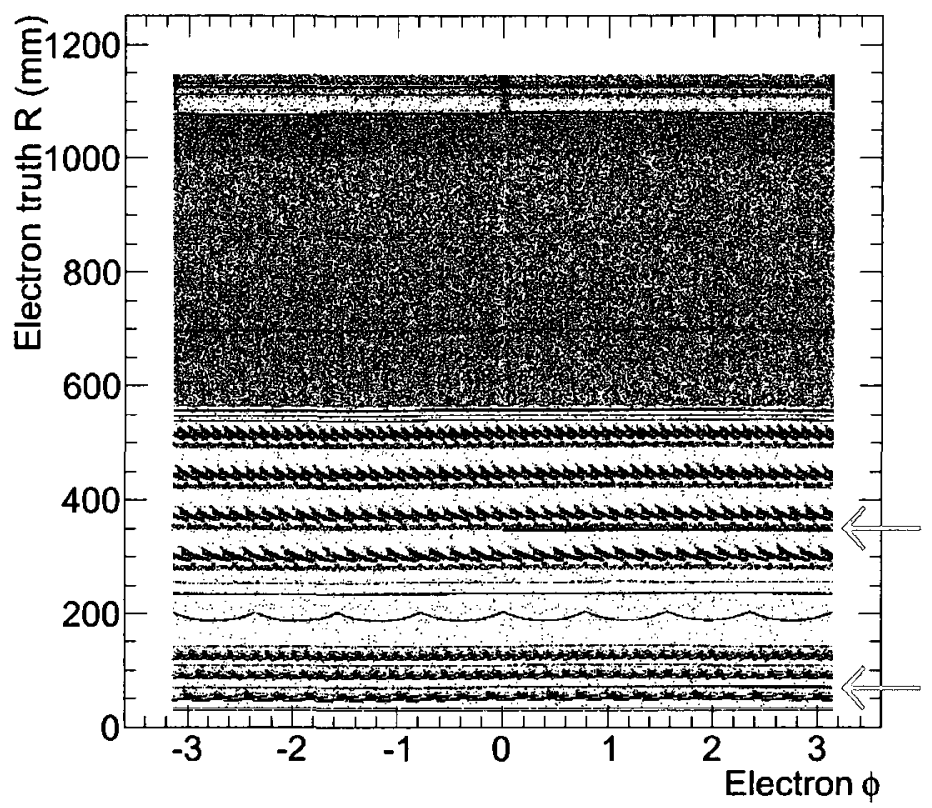

(a) Bremsstrahlung "x-ray" of the inner detector $(|\eta|<1)$.

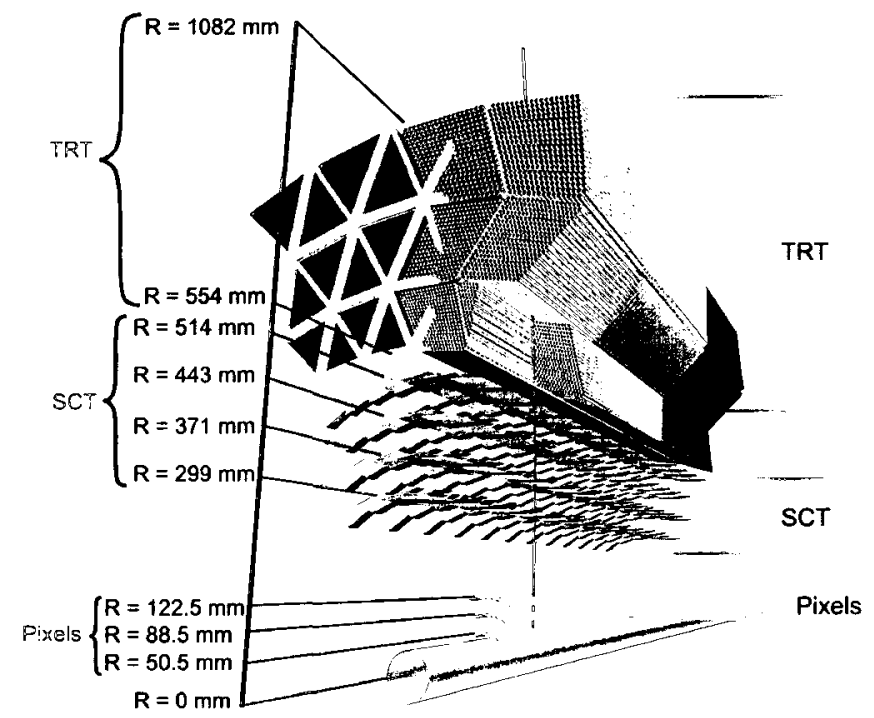

(b) Schematic of the inner detector.

FIGURE 4.29: (a) An "x-ray" of the inner detector obtained by plotting bremsstrahlung production truth coordinates. The arrows indicate the positions of extra material between the first and second pixel layers (bottom) and between the first and second SCT layers (top). (b) For comparison purposes, a schematic of the inner detector structure, from [13]. 
Chapter 4. Impact of Extra Material in Front of the EM Calorimeter and in the Inner Detector

the energy deposited in the calorimeter, it can be determined which regions of the inner detector (if any) affect the longitudinal energy deposition.

Figure 4.30 illustrates how the number of bremsstrahlung events can be used to locate material in the detector. The peaks in the approximate region $0 \mathrm{~mm}<$ $R<550 \mathrm{~mm}$ correspond to the pixel and SCT layers, and the solid mass at roughly $R>550 \mathrm{~mm}$ corresponds to the TRT (compare to Figure 4.29 (b)). In Figure 4.30 (b), three arrows indicate new peaks where extra material in the pixels, in the SCT barrel and between the SCT and TRT endcap has resulted in an increased number of bremsstrahlung events. For the purposes of this work, only the region $|\eta|<1$, which includes the extra material in the pixels, the SCT and between the TRT barrel and endcap and excludes the material between the SCT and the TRT endcap (refer to Figure 4.5), will be considered.

\subsubsection{Impact on Electron $E / p$}

The $E / p$ ratio of electrons in the detector should be equal to 1 ; however, the occurrence of bremsstrahlung in the inner detector can affect this ratio. When bremsstrahlung 
Chapter 4. Impact of Extra Material in Front of the EM Calorimeter and in the Inner Detector

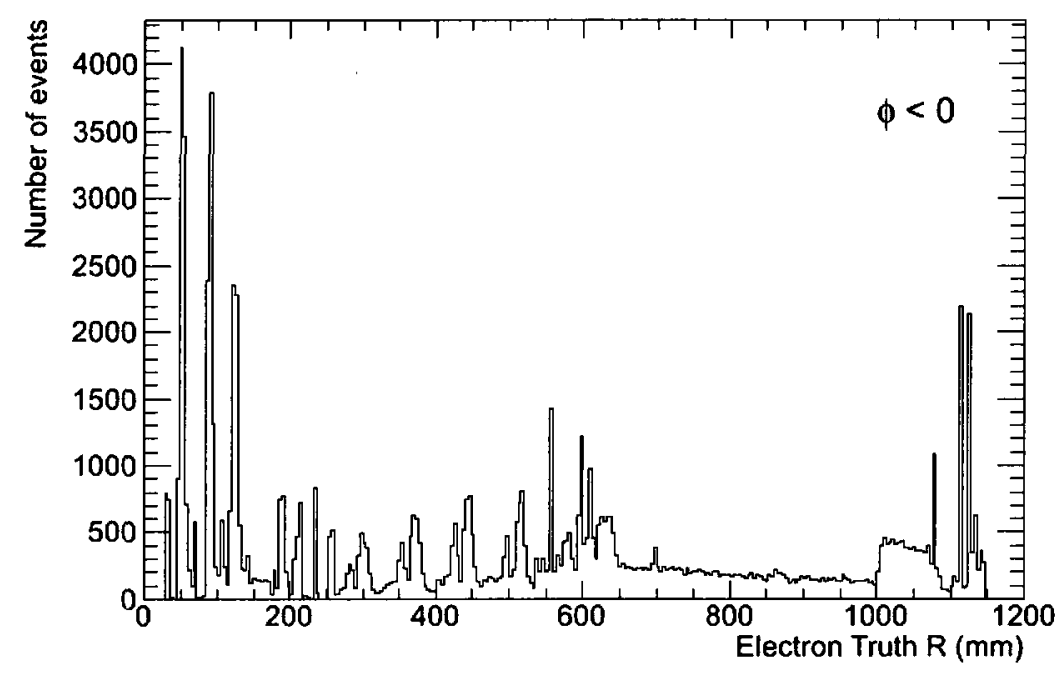

(a) Reference hemisphere.

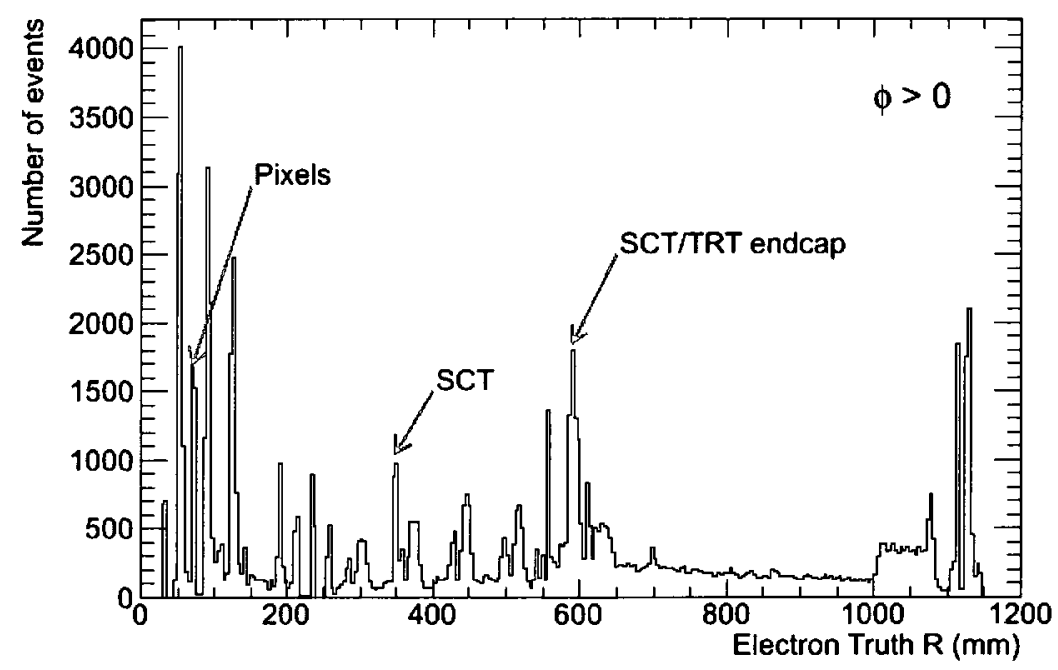

(b) Distorted hemisphere.

FiguRE 4.30: Monte Carlo truth $R$ coordinates of bremsstrahlung events occurring in the inner detector. The arrows indicate the locations of extra material in the distorted hemisphere. 
Chapter 4. Impact of Extra Material in Front of the EM Calorimeter and in the Inner Detector

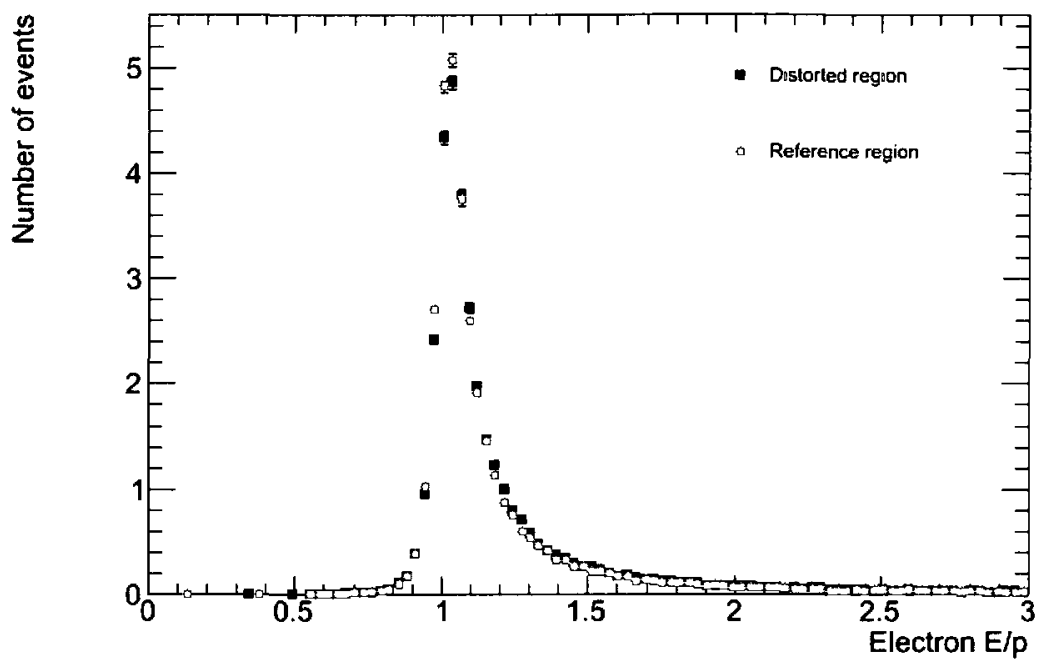

FIGURE 4.31: Normalized electron $E / p$ distribution. The distorted and reference regions refer to the regions with and without extra material in the inner detector, as given in Table 4.2 for $-1<\eta<0$.

the electron as it continues on its way will be lower than if bremsstrahlung had occurred later or not at all, causing the $E / p$ ratio to be greater than 1 . As shown in Figure 4.31, this effect results in a tail in the $E / p$ distribution.

In order to determine the impact of the extra inner detector material on the shape of the $E / p$ distribution, the fraction of events in the tail of the distribution was plotted for the distorted and reference regions of the inner detector (given in Table 4.2). Two definitions of the tail were used; first, a relatively generous cut selecting the events with $E / p>1.5$, and second, a stricter cut to select events with $E / p>2$. The resulting plots, shown in Figure 4.32, indicate that the fraction of events in the tail does indeed display some sensitivity to increased material; i.e. the 
Chapter 4. Impact of Extra Material in Front of the EM Calorimeter and in the Inner Detector

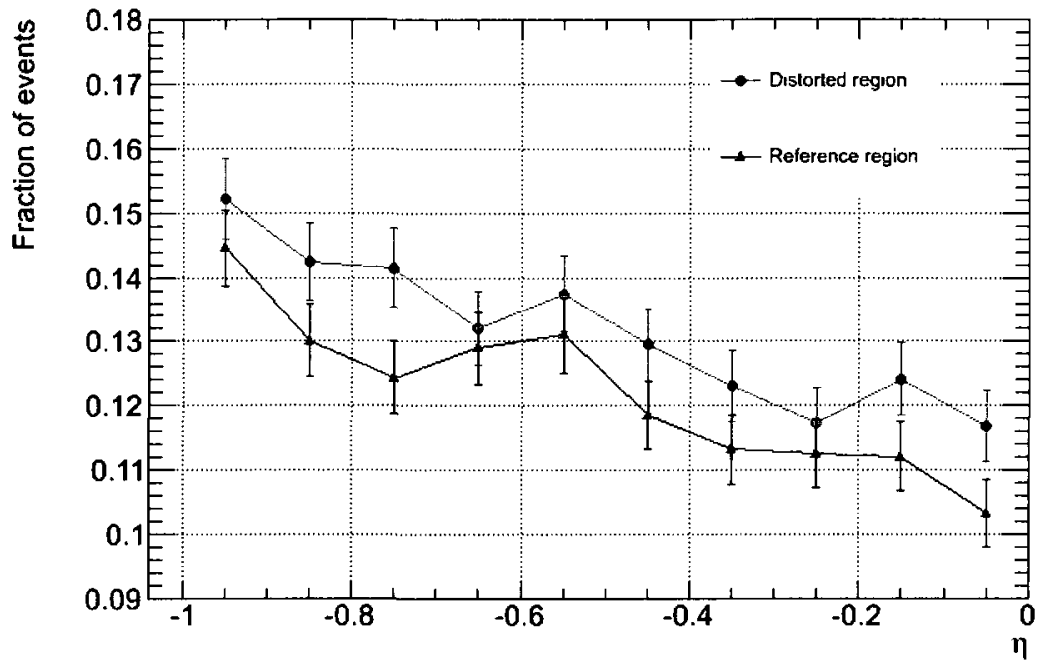

(a) Fraction of events with $E / p>1.5$.

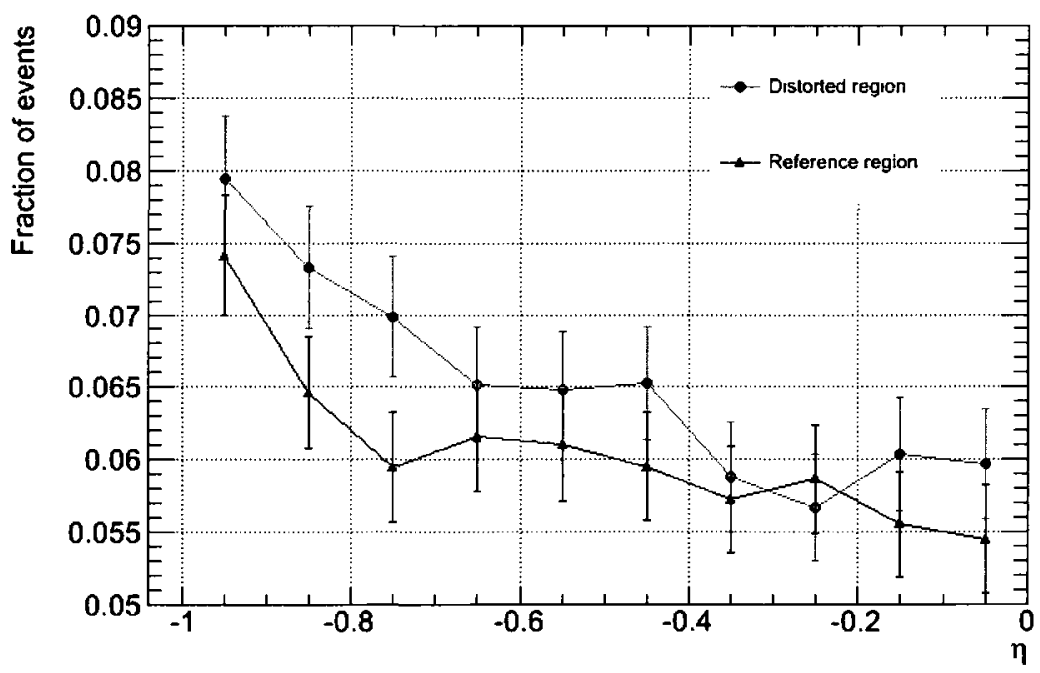

(b) Fraction of events with $E / p>2$.

FIGURE 4.32: (a) Fraction of events with $E / p>1.5$. (b) Fraction of events with $E / p>2$. The distorted and reference regions correspond to the regions with and without extra material in the inner detector, as given in Table 4.2. 
Chapter 4. Impact of Extra Material in Front of the EM Calorimeter and in the Inner Detector

fraction increases with $\eta$. The fraction of events in the tail is also systematically higher for the distorted region than for the reference region.

\subsubsection{Impact on Energy Deposited in the Calorimeter}

In order to determine the impact of the extra inner detector material on the energy deposited in each layer of the calorimeter, the estimator $R_{I D}$ was calculated using the specific definition of Equation 4.1

$$
R_{I D}=\frac{\left\langle x\left(\frac{\pi}{2}<\phi<\pi\right)\right\rangle}{\left\langle x\left(-\pi<\phi<-\frac{\pi}{2}\right)\right\rangle} ; \eta<0,
$$

where $x$ represents the fraction of energy deposited in the PS, S1, S2 or S3. In order to focus on extra material in a particular subdetector, additional cuts (given in Table 4.4; compare with Figure 4.30) were used, corresponding to the approximate Monte Carlo truth $R$-coordinate of the location where the bremsstrahlung events occurred. In the case of the TRT, the range in $R$ was expanded to cover the entire subdetector in order to include extra material in the barrel-endcap crack.

When calculated for the inner detector as a whole, i.e. without making any cuts on the $R$ coordinate, $R_{I D}$ was found to be consistent with unity at low values of $\eta$, as shown in Figure 4.33, indicating that this variable has little sensitivity to extra material in the pixels and in the SCT. At roughly $\eta \approx-0.7$, however, a noticeable 
Chapter 4. Impact of Extra Material in Front of the EM Calorimeter and in the Inner Detector

\begin{tabular}{l||c|r} 
Subdetector & Lower limit $(\mathrm{mm})$ & Upper limit $(\mathrm{mm})$ \\
\hline \hline Pixels & 66 & 112 \\
\hline SCT & 340 & 400 \\
\hline TRT & 563 & 1066
\end{tabular}

TABLE 4.4: Approximate location in $R$ of extra inner detector material.

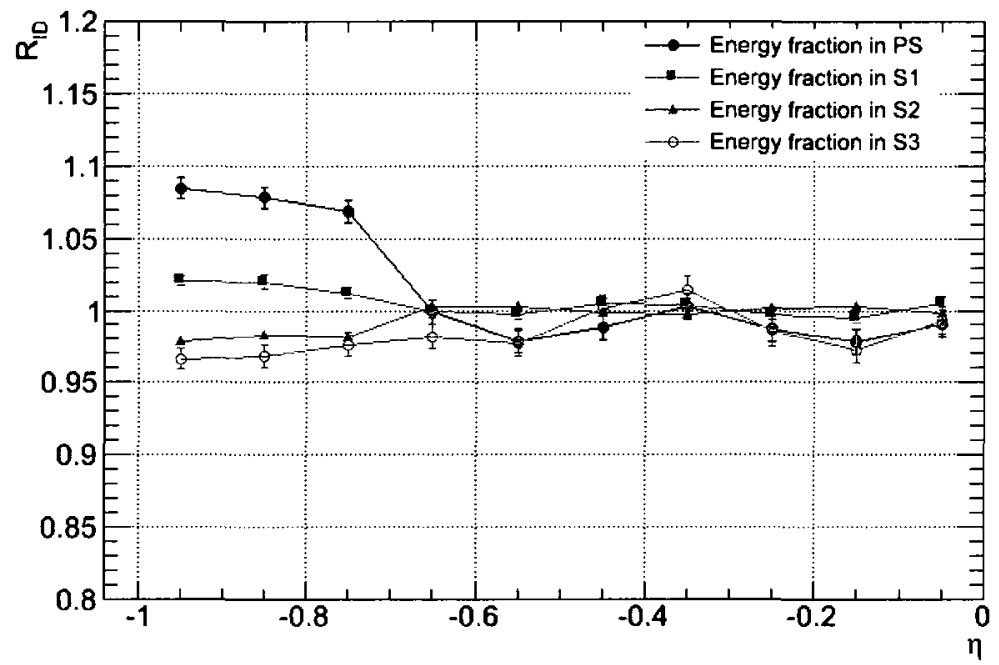

FIGURE 4.33: $R_{I D}$ for the energy fraction in each calorimeter layer, calculated using Equation 4.6.

deviation was observed, with $R_{I D}>1$ for the PS and S1 and $R_{I D}<1$ for S2 and S3. When this variable was calculated with the requirement that the $R$ value of a bremsstrahlung event occurred in the range of the pixels and for the SCT as defined in

Table $4.4, R_{I D}$ was consistent with unity for each layer of the calorimeter, indicating that the longitudinal energy deposition in the calorimeter is not significantly affected by bremsstrahlung in these two subdetectors. 
Chapter 4. Impact of Extra Material in Front of the EM Calorimeter and in the Inner Detector

The starting point of the deviation of $R_{I D}$ from unity $(\eta \approx-0.7)$ coincides with a dramatic increase in amount of material in the inner detector, as well as an increase in the proportion of material located in the distorted region (recall Figure 4.3). The bulk of this material is located near the TRT barrel-endcap interface. When $R_{I D}$ is calculated for the TRT region defined in Table 4.4 , the deviation at $\eta \approx-0.7$ once again becomes apparent, albeit to a lesser extent due to statistical fluctuations owing to the reduced number of events in this region.

$R_{I D}$ was also calculated for the new sensitive variable, $E_{1} / E_{2}$ for two general classes of events. The first class consisted of events involving "hard" bremsstrahlung, i.e. the Monte Carlo truth information indicated that the electron lost at least $15 \%$ of its energy in the form of bremsstrahlung in the inner detector. The second class consisted of "soft" bremsstrahlung events, in which the electron lost less than $15 \%$ of its energy in this manner; this class also takes into account events in which bremsstrahlung did not occur. Figure 4.34 suggests that $R_{I D}$ is consistent with unity for events in which soft or no bremsstrahlung events occured. On the other hand, an increase in $R_{I D}$ at $\eta \approx-0.7$ is apparent for events with hard bremsstrahlung, indicating that $E_{1} / E_{2}$ is somewhat sensitive to extra inner detector material as long as hard bremsstrahlung events occur.

This effect may be explained by Figure 4.35, which shows the average number of bremsstrahlung per primary electron as a function of $\eta$, obtained using the ATLAS 
Chapter 4. Impact of Extra Material in Front of the EM Calorimeter and in the Inner Detector

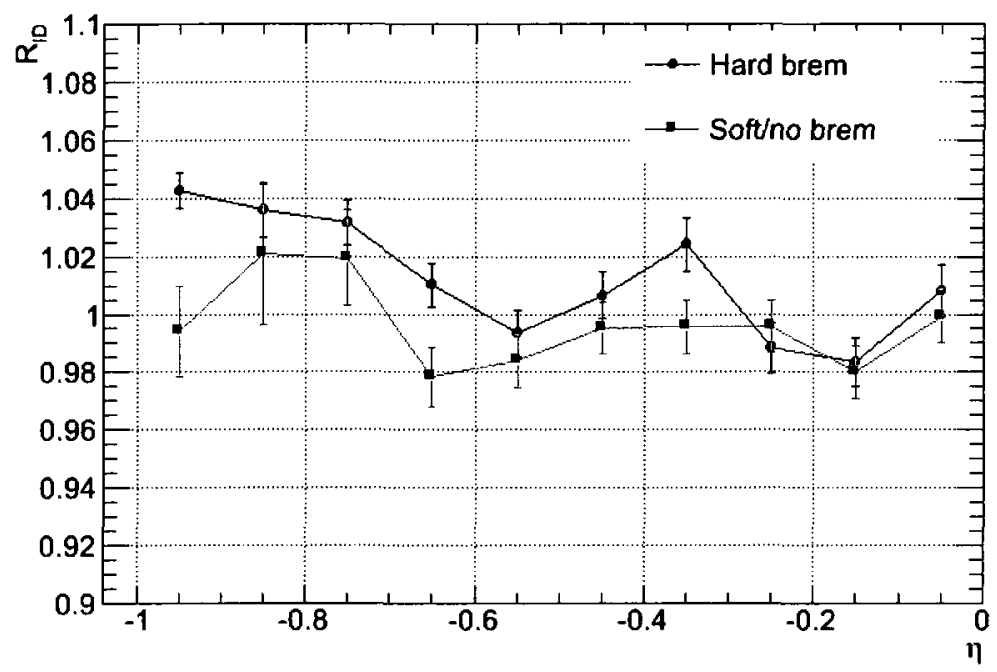

FIgURE 4.34: $R_{I D}$ as a function of $\eta$ for $E_{1} / E_{2}$, calculated for both hard and soft bremsstrahlung events.

Monte Carlo truth information. The number of bremsstrahlung is strongly correlated to the amount of material in the inner detector, which can be seen by comparing the shape of Figure 4.35 with that of Figure 4.3. Even at low $|\eta|$, where electrons must traverse relatively little material, the average number of bremsstrahlung per electron is greater than zero. For example, at $\eta \approx 0$, the average electron emits approximately three bremsstrahlung photons. Very few events yield no bremsstrahlung, especially at high $|\eta|$; this is reflected in the larger statistical errors on $R_{I D}$ for soft bremsstrahlung events in Figure 4.34. As mentioned previously, the proportion of material in the distorted hemisphere increases dramatically at high values of $|\eta|$, and so the effect of the nominal material increase in this same $|\eta|$ range cancels out in the calculation of 
Chapter 4. Impact of Extra Material in Front of the EM Calorimeter and in the Inner Detector

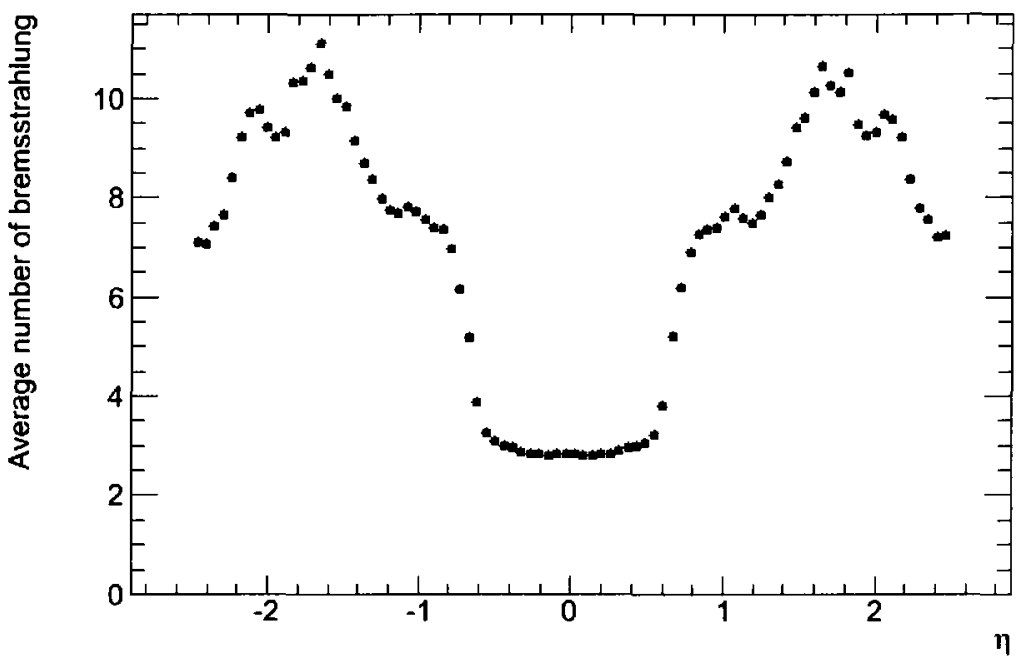

FIGURE 4.35: The average number of bremsstrahlung per event as a function of $\eta$.

$R_{I D}$, leaving only the effect of the extra material increase in the final result.

\subsection{Summary}

The method of Hubaut et al. has been shown to be a valid means of determining the sensitivity of electromagnetic shower variables to extra material in front of the calorimeter. Furthermore, it has been demonstrated that a very simple calculation may be used to predict the sensitivity of shower variables to increased amounts of material, yielding results consistent with those of the 2004 combined test beam. A new variable, $E_{1} / E_{2}$, while not as sensitive to extra material as was originally hoped, still has a high degree of statistical significance and is expected to be more robust than 
Chapter 4. Impact of Extra Material in Front of the EM Calorimeter and in the Inner Detector

any of the individual layer variables due to its sensitivity to the shower development in the first and second layers of the calorimeter.

The uncertainties on the results shown here are strictly statistical in nature.

While some attempts were made to give a rough estimate of the systematics associated with the results (see Figure 4.22, Figure 4.26 and Figure 4.28), the systematic errors can only be fully understood when the first LHC data arrive and the calibration of the calorimeter is verified. 


\section{Chapter 5}

\section{${ }_{1 \infty 00}$ Summary and Conclusions}

The ATLAS electromagnetic barrel calorimeter is a lead-liquid argon sampling calorimeter which utilizes a unique accordion structure in order to provide $\phi$-symmetry and hermetic coverage over a broad region in $|\eta|$. It will measure the energy deposited by electromagnetic showers initiated by electrons, positrons and photons resulting from

both Standard Model and new physics processes. During the early stages of operation, well-known Standard Model processes, including the decay $W \rightarrow e \nu$, will be studied in order to calibrate the detector and perform high-precision measurements of Standard Model processes. It is important that the location and amount of any unexpected extra material in front of the calorimeter be pinpointed as early as possible, as extra material can affect the measured shower shapes and energy deposition, thereby influencing the energy calibration of the detector. 
A method [16] to determine the sensitivity of shower variables to extra material using high $p_{T}$ electrons has been verified and expanded. An official ATLAS Monte Carlo sample was used, featuring a distorted, misaligned detector geometry in which various fractions of a radiation length of extra material had been simulated in the inner detector, in the cryostat and between the PS and S1 layers of the calorimeter. For comparison purposes, a reference region, in which no extra material had been added, was also included. The sample contained 2.5 million electrons from $W \rightarrow e \nu$ decays, corresponding to roughly $475 \mathrm{pb}^{-1}$ of data at $10 \mathrm{TeV}$. Properties of electromagnetic showers initiated by these electrons, including the energy scale and resolution, the energy deposited in each layer of the calorimeter, the ratio $E_{1} / E_{2}$ and the electrons' $E / p$ were compared for the distorted and reference regions in order to disentangle the effect of material in a particular region.

It was found that, in general, the presence of extra material in front of the calorimeter resulted in an increased fraction of energy being deposited earlier in the calorimeter (i.e. in the PS and S1) and a decreased fraction of energy being deposited later (i.e. in S2 and S2). This indicated that electromagnetic showers started earlier when extra material existed, in accordance with theory. The effect of extra material in the inner detector on the energy reconstruction was investigated, and it was found that the energy scale was underestimated by up to $2 \%$ and the energy resolution was degraded by up to $0.4 \%$, in agreement with the results of the original study. 
Once the method had been validated, it was expanded in order to quantify the sensitivity of the shower variables to extra material. A simple back-of-the-envelope calculation was used for this purpose to determine that the first and second layers of the calorimeter, for which this method gives the most reliable results, are sensitive to an increase of approximately $1 \%-5 \%$ of a radiation length of material in the cryostat, up to energies of approximately $80 \mathrm{GeV}$.

A new variable, $E_{1} / E_{2}$, which combines data from the first and second layers of the calorimeter, was tested in order to determine if it could be used as a more sensitive and/or robust variable. It was determined that this variable displays a degree of sensitivity greater than that of the individual layer variables. The results obtained for this variable are in excellent agreement with the results of the 2004 combined test beam, thus validating the use of the back-of-the-envelope method.

In order to gauge the feasibility of these studies during early running of the LHC, progressively smaller datasets were used to repeat the calculations until statistical fluctuations overshadowed the results. It was found that approximately 500,000 events, corresponding to roughly $160 \mathrm{pb}^{-1}$ at $10 \mathrm{TeV}$, should be sufficient to be sensitive to the amount of extra material added in this Monte Carlo simulation. This value is within the limits of the approximately $200 \mathrm{pb}^{-1}$ of data expected to be taken during the 2009 - 2010 run. 
Finally, bremsstrahlung truth information was used as a tool to "x-ray" the inner detector in an attempt to correlate the longitudinal energy deposition to material in a particular region of the inner detector. It was found that the measured momentum of electrons emitting bremsstrahlung photons early in the inner detector was lower than in the case of bremsstrahlung events occurring later or not at all, resulting in a value of $E / p>1$, and that the fraction of events with $E / p>1$ had some sensitivity to increased amounts of material. Extra material in the pixels and in the SCT was not found to have a significant impact on the fraction of energy deposited in each layer of the calorimeter, but there was some indication that these variables were affected by material near the TRT barrel-endcap interface. The new variable, $E_{1} / E_{2}$ was found to be sensitive to extra inner detector material in this region, but only in the case where hard bremsstrahlung photons were emitted.

This work assumes that the location of extra material in the detector is known precisely, which enables the comparison of the distorted and reference regions. The choice of the location of the extra material in this particular Monte Carlo sample was merely illustrative of where such material could be located. The official ATLAS Monte Carlo samples reflect the most up-to-date knowledge of the material composition and distribution, but this information can only be verified when the first data are taken during the 2009-2010 run. During this period, careful comparison of observed Standard Model processes, including $W \rightarrow e \nu$ decays, with the ATLAS Monte Carlo 
data will be of great importance in order to ensure that the calorimeter is properly calibrated. If a shower variable measured in a given region of the detector differs significantly from the expected value (according to the Monte Carlo predictions), this will serve as an indicator that this particular region contains extra material not accounted for in previous estimates of the material description, and the calibration may then be updated to reflect this difference. 


\section{References}

[1] J. J. Thomson. Cathode rays. Philosophical Magazine, 44:293, 1897.

[2] S. Abachi et al. Search for high mass top quark production in $p \bar{p}$ collisions at $1110 \sqrt{s}=1.8 \mathrm{TeV}$. Physical Review Letters, 74:2422-2426, 1995.

[3] F. Abe et al. Observation of top quark production in $p \vec{p}$ collisions with the Collider Detector at Fermilab. Physical Review Letters, 74:2626-2631, 1995.

[4] C. Amsler et al. (Particle Data Group). Review of particle physics. Physics Letters $B, 667(1), 2008$.

${ }_{1115}$ [5] D. Griffiths. Introduction to Elementary Particle Physics. Wiley-VCH, 2004.

[6] S. L. Glashow. Partial-symmetries of weak interactions. Nuclear Physics, 22: $579-588,1961$.

[7] S. Weinberg. A model of leptons. Physical Review Letters, 19:1264-1266, 1967. 
[8] A. Salam. Weak and electromagnetic interactions. In N. Svartholm, editor, Elementary Particle Theory, page 367. Almquist and Wiksells, 1969.

[9] P. W. Higgs. Broken symmetries, massless particles and gauge fields. Physics Letters, 12:132-133, 1964.

[10] P. W. Higgs. Broken symmetries and the masses of gauge bosons. Physical Review Letters, 13:508-509, 1964.

[11] ALEPH Collaboration, DELPHI Collaboration, L3 Collaboration, OPAL Collaboration, The LEP Working Group for Higgs Boson Searches. Search for the Standard Model Higgs boson at LEP. Physics Letters B, 565:61-75, 2003.

[12] The Tevatron New-Phenomena and Higgs Working Group. Combined CDF and $\mathrm{D} \varnothing$ upper limits on Standard Model Higgs-boson production with up to $4.2 \mathrm{fb}^{-1}$ of data. FERMILAB-PUB-09-060-E, 2009.

[13] G. Aad et al. The ATLAS Experiment at the CERN Large Hadron Collider. JINST, 3(S08003), 2008.

[14] The LEP Collaborations: ALEPH, DELPHI, L3, OPAL and the LEP Electroweak Working Group. A combination of preliminary electroweak measurements and constraints on the Standard Model. CERN-PH-EP/2006-042, 2006. 
[15] The LEP Collaborations: ALEPH, DELPHI, L3, OPAL and the LEP Electroweak Working Group. Precision electroweak measurements and constraints on the Standard Model. CERN-PH-EP/2007-039, 2007.

[16] F. Hubaut et al. Study of material in front of the EM calorimeter with high $\mathrm{p}_{T}$ 1140 electron shower shapes and tracks. ATL-PHYS-INT-2008-026, 2008.

[17] L. Evans and P. Bryant, editors. LHC machine. JINST, 3(S08001), 2008.

[18] P. Lefèvre and T. Pettersson, editors. The Large Hadron Collider: Conceptual design. CERN-AC-95-05 LHC, 1995.

[19] The ATLAS experiment, 2009. URL http://atlas.ch.

[20] ATLAS Inner Detector Community. ATLAS inner detector: Technical design report, 1. CERN-LHCC-97-016, 1997.

[21] ATLAS Inner Detector Community. ATLAS inner detector: Technical design report, 2. CERN-LHCC-97-017, 1997.

[22] ATLAS LARG Unit. ATLAS liquid argon calorimeter: Technical design report. CERN-LHCC-96-41, 1996.

[23] ATLAS Tile Calorimeter Collaboration. ATLAS tile calorimeter: Technical design report. CERN-LHCC-96-042, 1996. 
[24] ATLAS Muon Collaboration. ATLAS muon spectrometer: Technical design report. CERN-LHCC-97-022, 1997.

[30] G. Aad et al. Expected performance of the ATLAS Experiment: Detector, trigger and physics. CERN-OPEN-2008-020, 2008.

[31] ATLAS Collaboration. ATLAS calorimeter performance: Technical design report. CERN-LHCC-96-40, 1997. 
[32] B. Aubert et al. Development and construction of large size signal electrodes for the ATLAS electromagnetic calorimeter. Nuclear Instruments and Methods in Physics Research A, 539(3):558-594, 2005.

[33] M. L. Andrieux et al. Construction and test of the first two sectors of the ATLAS barrel liquid argon presampler. Nuclear Instruments and Methods in Physics Research A, 479(2-3):316-333, 2002.

[34] M. Delmastro. Electronic calibration of the ATLAS LAr calorimeters. May 2008. URL http://indico.cern.ch/materialDisplay.py?contribId= 0\&material Id=slides\&conf $I d=35889$.

[35] M. Aharrouche et al. Energy linearity and resolution of the ATLAS elctromagnetic barrel calorimeter in an electron test-beam. Nuclear Instruments and Methods in Physics Research A, 568(2):601-623, 2006.

[36] N. Kerschen. Performance of the ATLAS LAr barrel calorimeter in the 2004 combined test beam. XIII International Conference on Calorimetry in High Energy Physics, 2008. URL http://agenda.infn.it/conferenceOtherViews. py?view=standard\&amp; $\operatorname{conf} I d=352$.

[37] ATLAS Collaboration. Distorted material in CSC simulation, 2007. URL https : //twiki.cern.ch/twiki/bin/view/Atlas/DistortedMaterial.

[38] I. Wingerter-Seez. Private communication (to be published). 